Portland State University

PDXScholar

Spring 6-5-2013

\title{
The Role of Psycho-Sociocultural Factors in Suicide Risk Among Mong/Hmong Youth
}

TangJudy Vang

Portland State University

Follow this and additional works at: https://pdxscholar.library.pdx.edu/open_access_etds

Part of the Asian American Studies Commons, Asian Studies Commons, and the Race and Ethnicity Commons

Let us know how access to this document benefits you.

\section{Recommended Citation}

Vang, TangJudy, "The Role of Psycho-Sociocultural Factors in Suicide Risk Among Mong/Hmong Youth" (2013). Dissertations and Theses. Paper 1037.

https://doi.org/10.15760/etd.1037

This Dissertation is brought to you for free and open access. It has been accepted for inclusion in Dissertations and Theses by an authorized administrator of PDXScholar. Please contact us if we can make this document more accessible: pdxscholar@pdx.edu. 
The Role of Psycho-Sociocultural Factors in Suicide Risk Among Mong/Hmong Youth

\author{
by \\ TangJudy Vang
}

A dissertation submitted in partial fulfillment of the requirements for the degree of

\author{
Doctor of Philosophy \\ in \\ Social Work and Social Research
}

Dissertation Committee:

Vikki Vandiver, Chair

Laurie Powers

Keva Miller

Yer Thao

Portland State University

2013 
(C) 2013 TangJudy Vang 


\begin{abstract}
This study examined psychological, social, and cultural factors that can affect suicide risk among Mong/Hmong youth between the ages of 18 and 25. Emerging evidence suggests that Mong/Hmong youth are at an increased risk for suicide (Huang, Lee, \& Arganza, 2004; Jesilow \& Xiong, 2007). Additionally, initial findings and theories have suggested potential associations between Mong/Hmong youth suicide risk and intergenerational family conflict, ethnic identity, acculturation, depression, and spirituality. The seriousness of suicide risk among Mong/Hmong youth in this country has been overlooked for decades; therefore, the purpose of this study was to examine these associations with the hope that the findings would be beneficial in future efforts to reduce suicide risk among Mong/Hmong youth.

This research was a cross-sectional exploratory study that used a purposive sampling method in addition to snowball sampling. The sample consisted of 165 Mong/Hmong youth between the ages of 18 and 25 from three California academic institutions. Results indicated that of 165 respondents, 59\% ( $n=98)$ have had passing thoughts of suicide. There was a correlation between ethnic identity, intergenerational family conflict, depression, and spiritual beliefs. Furthermore, ethnic identity and intergenerational family conflict were significant predictors of depression. Lastly, depression and having a belief in Mong/Hmong traditional spiritual and healing practices were predictors of suicide risk among the sampled population.
\end{abstract}

Two open-ended protective factor questions were explored to encourage participants to reflect on their resilience to suicide by sharing how they responded to 
thoughts of ending their life and what helped them to overcome those thoughts. Five themes were identified as protective factors: (1) having the cognitive ability to understand how death affects loved ones; (2) optimism and having a positive orientation toward the future; (3) connectedness with family, friends, and community; (4) having a sense of selfworth; and (5) a social life.

Implications for social work practice and policy include the development, expansion and delivery of culturally appropriate mental health treatment services for young adults. This entails the incorporation of traditional Mong/Hmong mental health healing practices into western mental health treatment, ongoing clinical research to better understand the mental health needs of the Mong/Hmong young adult population, and educating and empowering the Mong/Hmong community to access the mental health system, thereby reducing the stigma associated with mental health and increasing access to treatment. 


\section{Acknowledgements}

My journey to the completion of this dissertation would not be complete without acknowledging the individuals who provided their endless support throughout my Ph.D. program. To my dissertation Chair, Dr. Vikki Vandiver and dissertation committee members, Drs. Laurie Powers, Keva Miller, Yer Thao, Daniel Coleman, and Stephanie Wahab - I thank each and every one of you for your patience in enduring this long academic path with me; your guidance was greatly appreciated. And last but not least, I'd like to thank Pauline Duffy for being the backbone to Portland State University School of Social Work Ph.D. Program. Despite the distance, you've continued to cheer us on.

To my husband, Cheng Kue, and beautiful children, Tyler N. Kue and Evelyn K. Kue, thank you for the ongoing motivation, support, and love. It excites me to know that we completed this journey together. And lastly, to my parents, and siblings, your continued support and encouragement means the world to me. Thank you ALL for not giving up on me, even when I doubted myself. 
Table of Contents

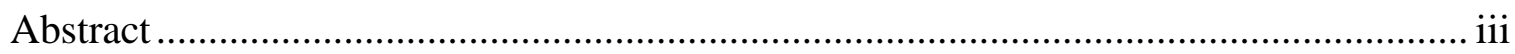

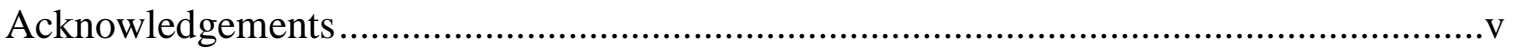

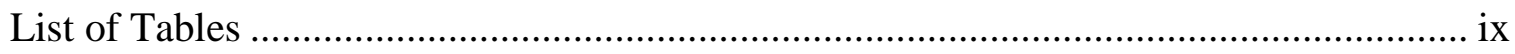

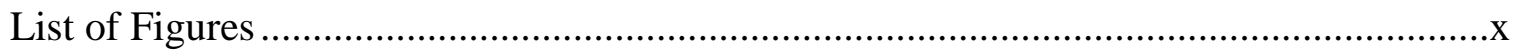

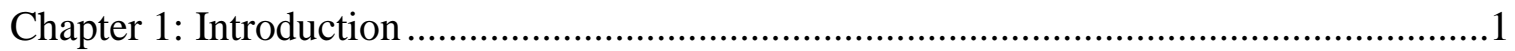

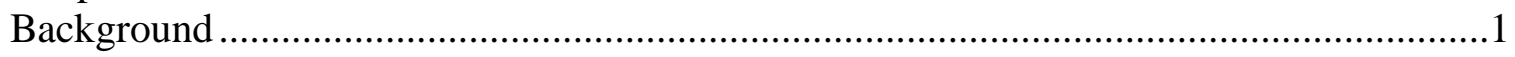

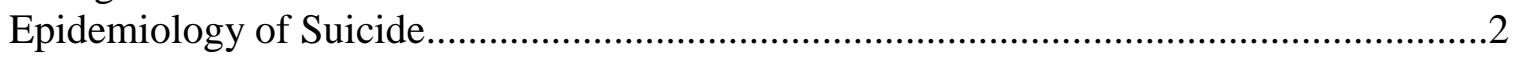

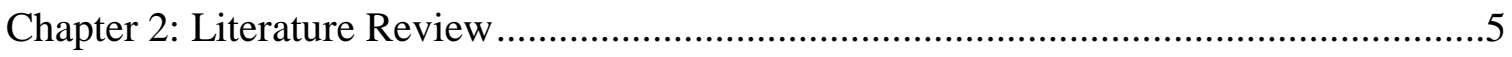

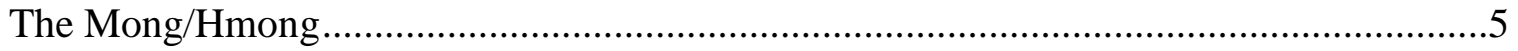

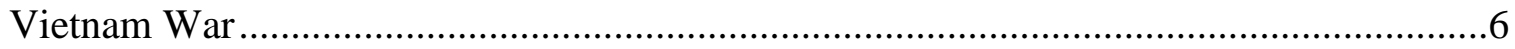

Traditional Mong/Hmong Family System ............................................................

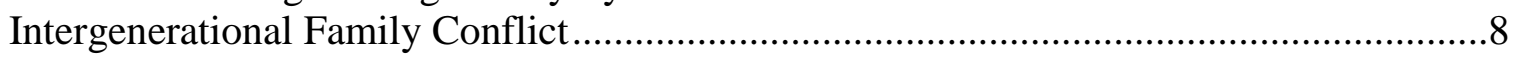

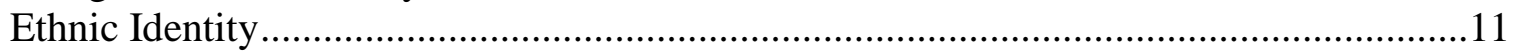

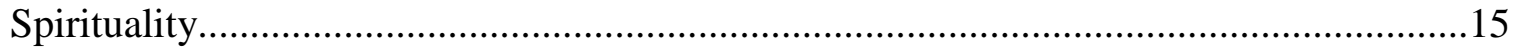

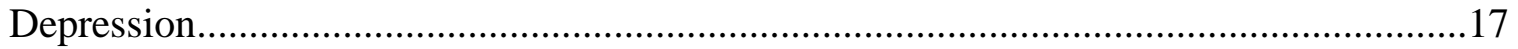

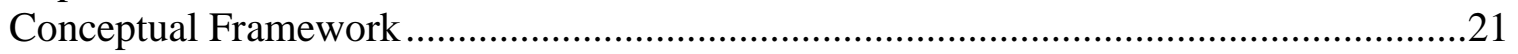

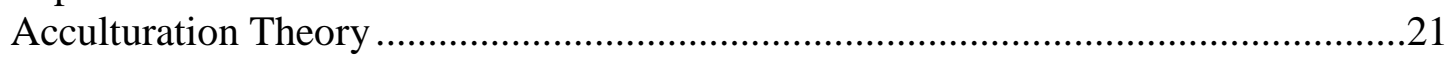

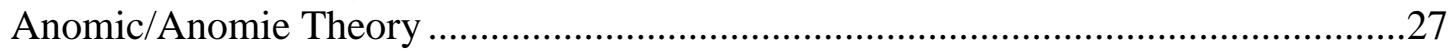

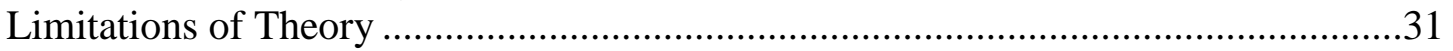

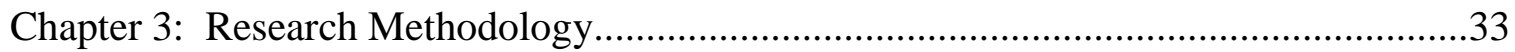

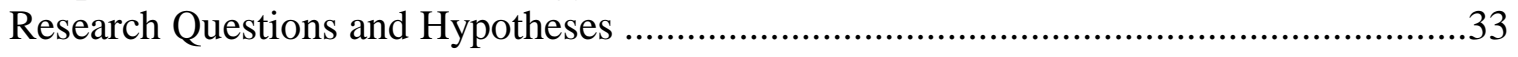

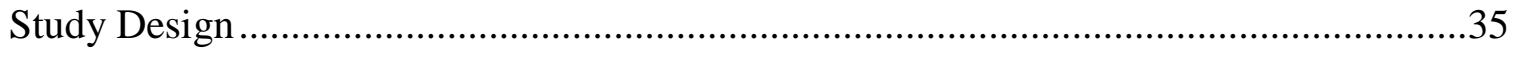

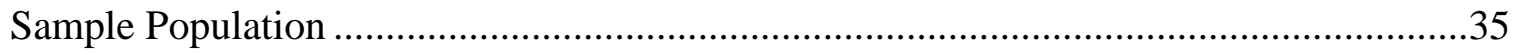

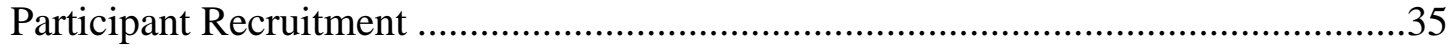

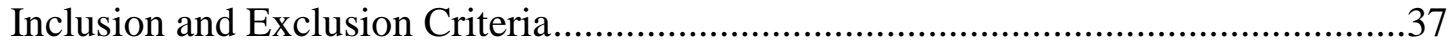

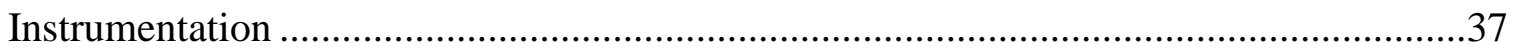

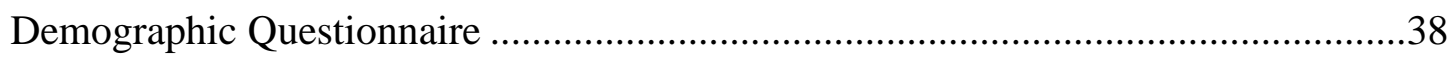

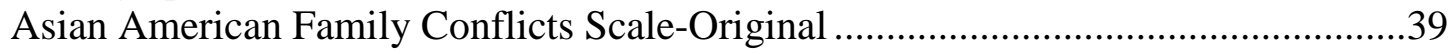

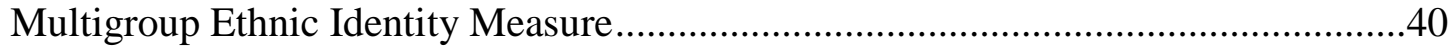

Suinn-Lew Asian Self-Identity Acculturation Scale...............................................41

Zung Self-Rating Depression Scale ................................................................. 41

Suicide Behaviors Questionnaire-Revised.........................................................42

Mong/Hmong Kev Ntseeg Instrument ............................................................43

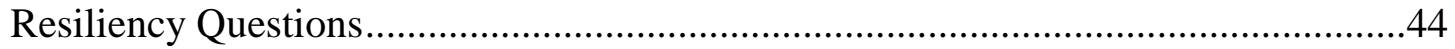

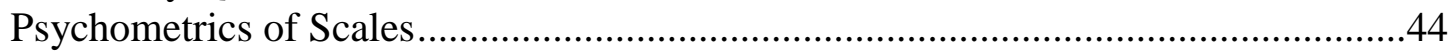

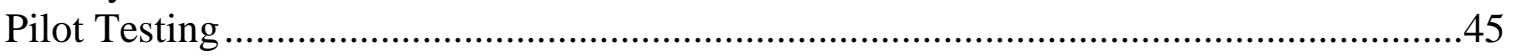




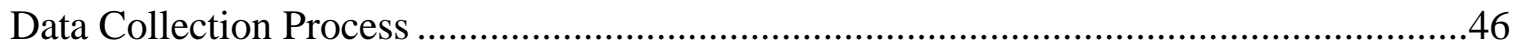

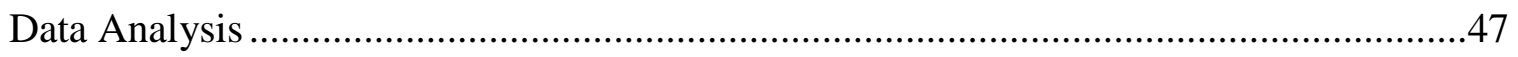

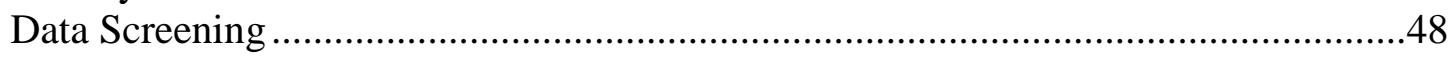

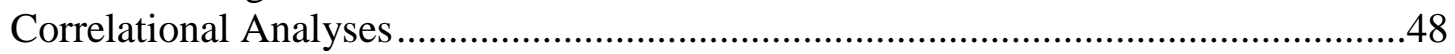

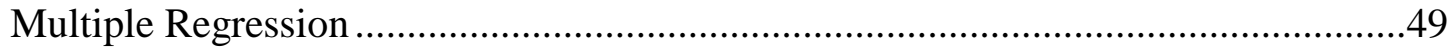

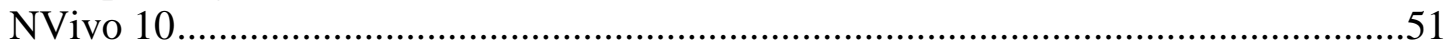

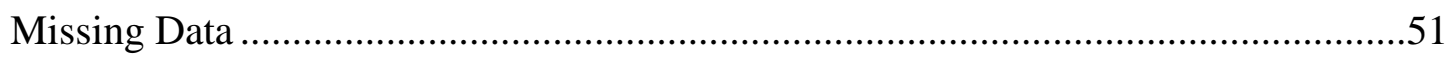

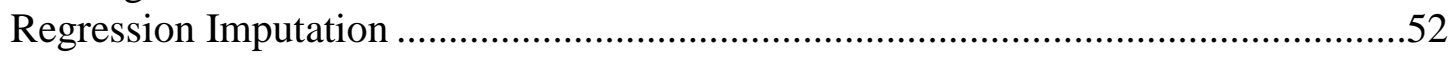

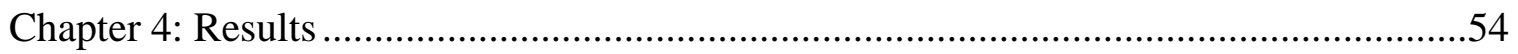

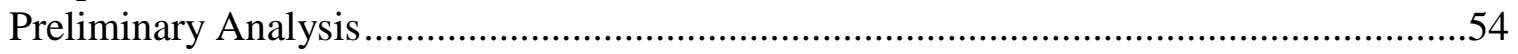

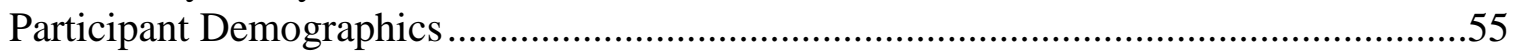

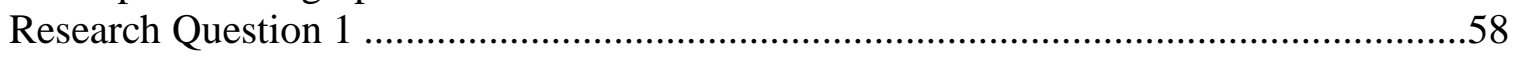

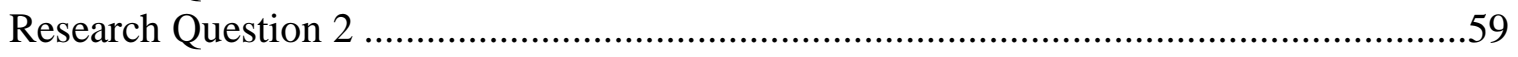

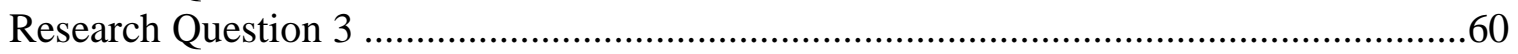

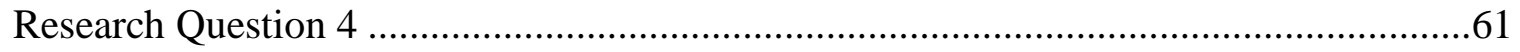

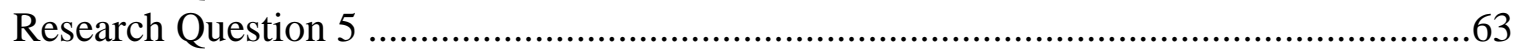

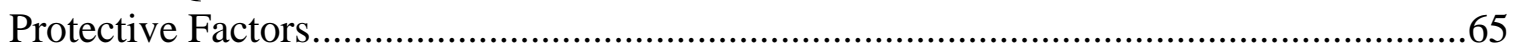

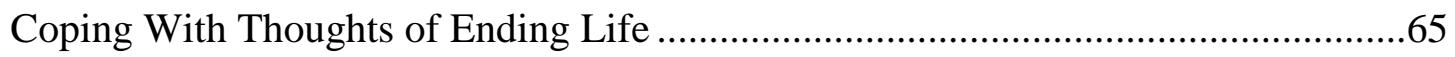

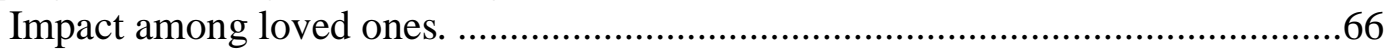

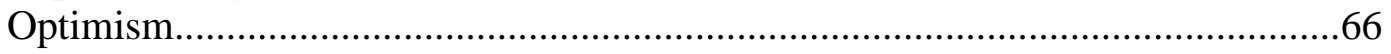

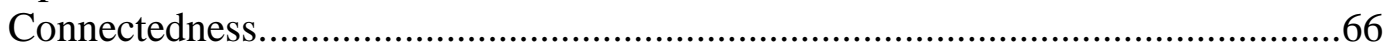

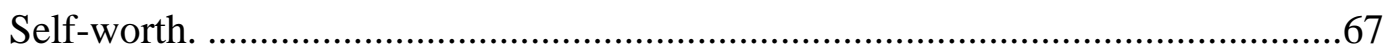

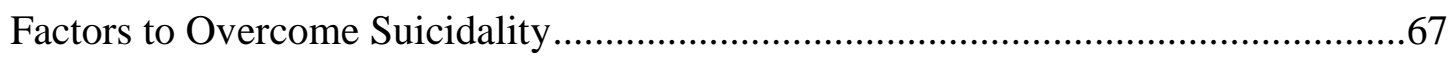

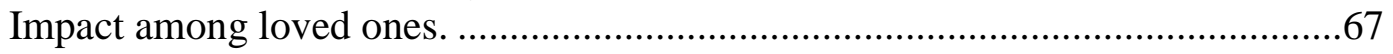

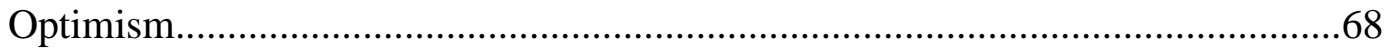

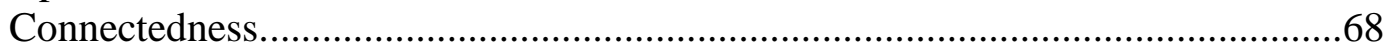

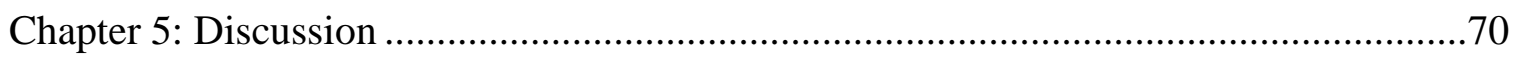

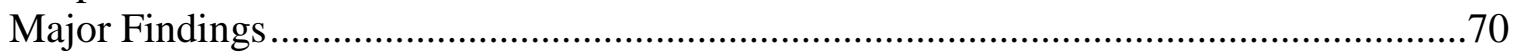

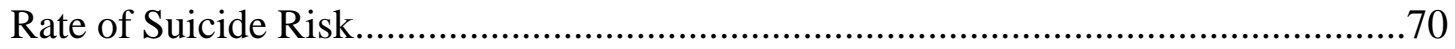

Correlation Between Depression and Ethnic Identity ........................................... 71

Correlation Between Healing Belief and Ethnic Identity ..................................... 71

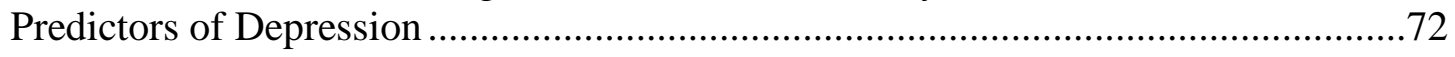

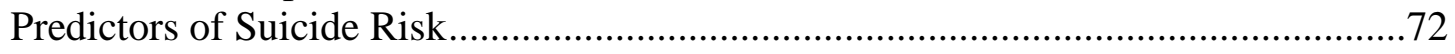

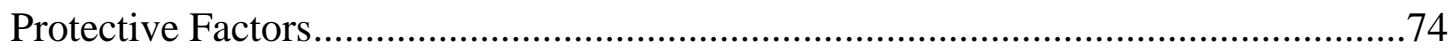

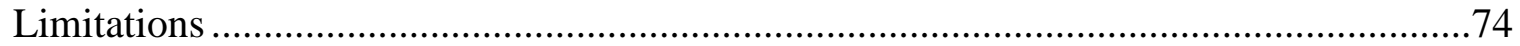

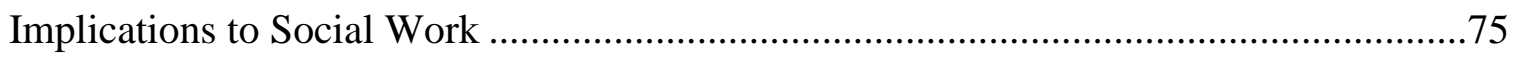

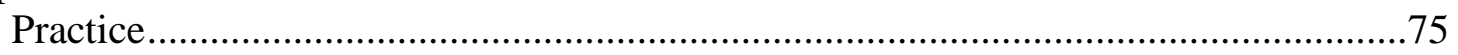

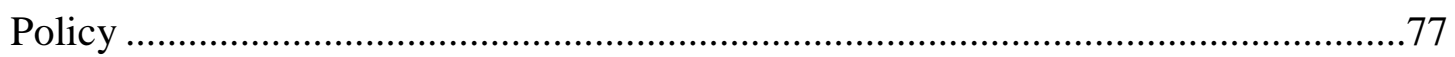

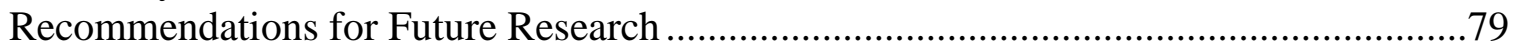

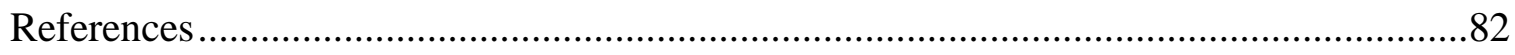

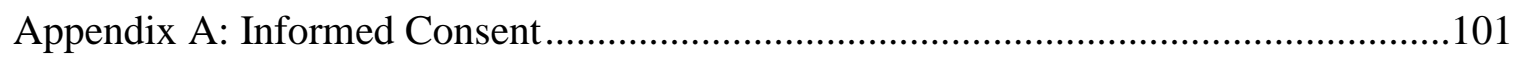


Appendix B: Demographic Questionnaire

Appendix C: Asian American Family Conflict Scale - Original ...................................106

Appendix D: Multigroup Ethnic Identity Measure ...................................................107

Appendix E: Suinn-Lew Asian Self-Identity Acculturation Scale ................................109

Appendix F: Zung Self-Rating Depression Scale ....................................................114

Appendix G: SBQ-R Suicide Behavior Questionnaire Revised ...................................115

Appendix H: Mong/Hmong Kev Ntseeg Instrument ................................................116

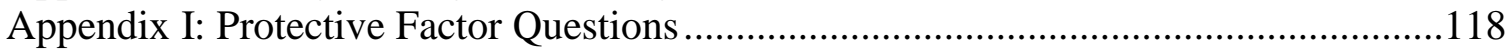

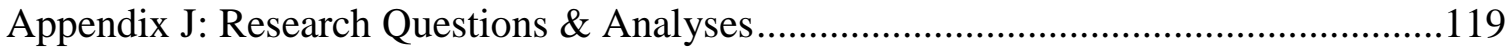

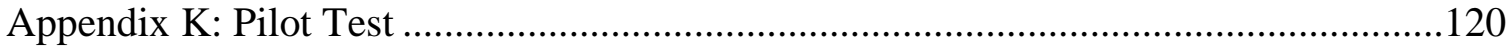

Appendix L: Recruitment/Data Collection Process .....................................................121 
List of Tables

1. Demographic Characteristics of Participants ......................................................56

2. Lifetime Suicide Ideation and/or Suicide Attempts .............................................58

3. Pearson Product-Moment Correlations Between Ethnic Identity and Depression .......59

4. Pearson Product-Moment Correlations Between Intergenerational Family Conflict, Traditional \& Spiritual Healing Beliefs, and Ethnic Identity

5. Pearson Product-Moment Correlations Between Depression, Acculturation, Ethnic Identity, and Intergenerational Family Conflict

6. Multiple Regression Analyses for Variable Predicting Depression among Mong/Hmong Youth $(\mathrm{N}=154)$

7. Pearson Product-Moment Correlations Between Suicide Risk, Ethnic Identity, Intergenerational Family Conflict, Depression, Family Household Size, and Mong/Hmong Traditional \& Spiritual Healing Beliefs

8. Multiple Regression Analyses for Variables Predicting Suicide Risk among Mong/Hmong Youth $(\mathrm{N}=158)$ 
List of Figures

1. Variations in acculturative stress as a function of phase and mode of acculturation

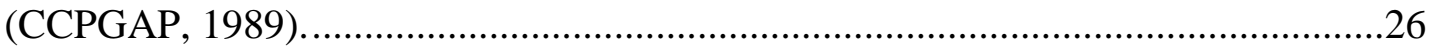




\section{Chapter 1: Introduction}

The Mong/Hmong are one of the more recent Southeast Asian ethnic refugee groups to migrate to the United States, and are among the poorest immigrants with low rates of academic performance, high dependency on government assistance, and severe mental health issues (Su, Lee, \& Vang, 2005). Although the Mong and Hmong are from the same ethnic group and share similar historical backgrounds and values, they differ linguistically (e.g., dialect) and culturally (e.g., traditional clothing style; P. Thao \& Yang, 2004). However, the terms Mong and Hmong are used synonymously (i.e., Mong/Hmong) when referring to both groups (P. Thao \& Yang, 2004). The Mong speak the Mong Leng language, often referred to in western culture as the Green Mong although it is a derogatory term, and the Hmong speak the Hmong Der language, referred to in western culture as the White Hmong.

\section{Background}

The historical roots of the Mong/Hmong in the United States date to the mid1970s after the fall of Saigon. After immigrating to the United States, the majority of Mong/Hmong elders continued to maintain their cultural and traditional practices beyond the influences of the American culture (MacDonald, 1997). According to several acculturation research studies (Miller \& Lee, 2009; Sluzki, 1979; Su et al., 2005), parents and elders who immigrate into the United States are less likely to adapt to the host culture than are their children due to a cultural commitment to preserve their ethnic identity and cultural traditions. Many Asian American children whose parents immigrate into the United States are torn between maintaining their cultural identity while adapting to 
western culture (Su et al., 2005). This difficulty with balancing one's original culture and ethnic identity in the host country has resulted in intergenerational family conflict. For example, Greenberger and Chen (1996) and Uba (1994) suggest that the different rates of acculturation among immigrant families are correlated with serious mental health issues which include emotional distress and can result in domestic violence, gang involvement, and suicide.

\section{Epidemiology of Suicide}

The World Health Organization (2006) reported that in 2000, an estimated 877,000 people committed suicide, which equates to one suicide every 40 seconds. In the United States alone, approximately 30,000 Americans die each year from suicide (U.S. Department of Health and Human Services [DHHS], 1999). In 2010, suicide ranked as the 10th leading cause of death among Americans and the third leading cause of death among those aged 15 to 24 . It is estimated that among those aged 15 to 24,100 to 200 young adults have attempted suicide for every death that ended in suicidality (Centers for Disease Control and Prevention, 2012). Each year, suicide claims more lives than cancer, heart disease, and acquired immunodeficiency syndrome (AIDS).

Adolescent and young adult suicide rates vary according to race, gender, and economic circumstances (DHHS, 2001). According to the National Asian American Pacific Islander Mental Health Association (NAAPIMHA, 2007), Asian American youth are at the greatest risk for developing suicidal behavior and mental health illnesses. Furthermore, Asian American women between the ages of 15 and 24 have the highest rate of suicide when compared to Caucasian, African, and Mexican Americans (National 
Alliance for the Mentally Ill, 2003; NAAPIMHA, 2007). Racially and culturally, Asian Americans are a heterogeneous group with substantial intergroup variations in rates of suicide.

Within the last decade, the rates of Southeast Asian youth suicide attempts and completion of suicide, in particular, have increased (Huang et al., 2004; Jesilow \& Xiong, 2007; NAAPIMHA, 2007). According to a preliminary study conducted by the California Endowment (2006), the personal and social problems that Mong/Hmong youth are facing are intensifying as shown by increases in their rates of self-harm, such as cutting, substance abuse (37\%), and level of depression (58\%). These increases are likely due to a multitude of psycho-sociocultural factors, such as intergenerational family issues, peer issues, academic pressure, and cultural conflict. Although there is scarce research on the mental health of Asian Americans and their suicide rates, reports have indicated that Southeast Asian subgroups are at risk of developing increased emotional and behavioral problems as a result of cultural conflicts arising from family and community (Huang et al., 2004; DHHS, 1999).

According to the DHHS (1999), "more information is needed on suicide among subgroups of Asian Americans" (p. 115). More recently, youth suicide attempts and suicide completion among the Mong/Hmong population in the United States have significantly risen, yet there remains a lack of research on this phenomenon (NAAPIMHA, 2007; DHHS, 2002). For instance, from 1998 to 2001, eight Mong/Hmong youth in one California community committed suicide; four of the eight suicides occurred within a 6-month period (The California Endowment, 2006; Huang, 
2006; DHHS, 2002). These traumatic losses have resulted in Mong/Hmong families and communities experiencing a significant amount of mental and emotional distress. This social and cultural phenomenon of Mong/Hmong youth suicide attempts and the completion of suicide continues to be misunderstood by many Mong/Hmong parents and elders as they attempt to preserve their cultural traditions and values in this country (Jesilow \& Xiong, 2007).

Preliminary evidence suggests that Mong/Hmong youth may be at particular risk for suicide (Jesilow \& Xiong, 2007; Mouanoutoua, 2003; DHHS, 2002). As a result, there is an important need to increase the understanding of suicide in this group and develop preventive measures and interventions to deter future deaths from occurring. Existing studies on the Mong/Hmong population in this country reported that this ethnic group manifests more symptoms of depression than other Southeast Asian subgroups (Gong-Guy, 1987; Westermeyer, Tou, \& Neider, 1984); faces extreme intergenerational family conflict and differences in acculturation (Lee, Su, \&Yoshida, 2005; K. Yang, 2003); and reluctance to use western forms of health care treatment. Instead, Mong/Hmong individuals prefer traditional herbal therapies and healers over mental health care (e.g., Shaman/Shamanism; Cerhan, 1990; Fadiman, 1997; DHHS, 1999).

Given the traumatic historical account of the Mong/Hmong immigration into this country, the familial challenge in maintaining their cultural traditions in this multicultural society, and rise of youth suicide attempts, the aim of this study was to examine whether intergenerational family conflict, ethnic identity, acculturation, depression, and spirituality influence suicide risk among Mong/Hmong youth. 


\section{Chapter 2: Literature Review}

This chapter will describe and summarize the history, traditional family structure, and traditional spiritual and healing beliefs of the Mong/Hmong people. Literature on intergenerational family conflict, ethnic identity, spirituality, and depression are summarized to further explore how each of these impact suicide risk. Additionally, acculturation theory and anomie theory are examined to help explain the social problem of Mong/Hmong youth suicide risk.

\section{The Mong/Hmong}

The Mong/Hmong are a Southeast Asian ethnic subgroup with no country of origin. To date, the historical origins of the Mong/Hmong are unclear. With only folklore, theories, and myths to describe their historical roots, the Mong/Hmong are well known to anthropologists as strong and collective mountainous tribesmen who forcefully fought their way to become free from slavery and warfare. Because the written language of the Mong/Hmong was recently developed in the 1950s by French missionaries, the Mong/Hmong have no form of historical documents to trace their ancestries and country of origin (N. Yang, 1995).

The earliest archeological documents on the Mong/Hmong history indicate that the Mong/Hmong escaped China during the 19th century due to wars and persecution (Quincy, 1988; Y. J. Thao, 2006). The majority of the Mong/Hmong left southern China and settled in the mountains of Indochina (i.e., Laos, Thailand, Burma, and Vietnam), with the largest population in Laos. This migration to the highlands of Laos and 
surrounding countries of southern China allowed the Mong/Hmong to "preserve their cultural identity" and find peace (C. Thao, 1999, p. 13).

\section{Vietnam War}

The Mong/Hmong were secluded from other Southeast Asian subgroups and kept to themselves until the1960s, when the U.S. military recruited thousands of Mong/Hmong men and boys to help fight against communists during the Vietnam War (N. Yang, 1995). The Vietnam War, also known as the "Secret War of Laos," was fought during 1961 to 1973 (Bliatout, Downing, Lewis, \& Yang, 1988). In 1973, after the war claimed more than an estimated 30,000 Mong/Hmong soldiers, the United States withdrew. Chan (1994) estimated that at least $25 \%$ of the Mong/Hmong men and boys who were recruited by the U.S. military were killed during the war, while 17,000 Mong/Hmong troops and 50,000 Mong/Hmong vanished during the duration of the war.

After the war, the Mong/Hmong were abandoned by their American allies and left to survive on their own under the communist rule (Helzer, 1993). The war left the American public unaware of the Mong/Hmong involvement with the U.S. military. Many historians and Mong/Hmong refugees have indicated that it was the intent of the U.S. military and government to prevent the American public from learning about the coalitions formed between the Southeast Asian hill-tribes and the Americans during the war (Pfaff, 1995).

By 1975, communists took over Laos and thousands of Mong/Hmong fled the country and sought asylum in Thailand and surrounding countries (Quincy, 1988). The Mong/Hmong who remained in Laos or who were unable to escape were raped, held as 
prisoners, forced into slavery, or murdered by communists. Under the Refugee Act of 1980, thousands of Southeast Asian refugees, including the Mong/Hmong, were admitted into the United States and scattered across the country to diversify the refugee communities (Cao \& Novas, 1996; Pfaff, 1995).

Adjusting to western culture was and continues to be challenging for many first generation Mong/Hmong Americans as well as their children (Bliatout, 2003). The warfare and horrific experiences of fleeing Laos to neighboring countries were traumatizing and forced many Mong/Hmong to abandon family members and their homes. The displacement of Mong/Hmong families to different parts of the world has contributed to much emotional distress as this close-knit subgroup has struggled to maintain its cultural identity, ethnicity, and religious practices (Chan, 1994).

\section{Traditional Mong/Hmong Family System}

Family is highly valued within the Mong/Hmong culture and includes extended family members such as grandparents, uncles, aunts, and cousins from the same clan. A clan is defined by the husband's family surname. Immediate family members within a Mong/Hmong family usually consist of eight to 10 children; however, within the United States, economic disadvantage has decreased family size (McInnis, 1991). In traditional Mong/Hmong culture, married couples are encouraged to have a large family to help tend the farm and care for their parents once they become old and fragile. The immediate family is the core unit of labor. Family needs have priority over individual needs. Traditional Mong/Hmong families are patriarchal—men hold the authority and women are excluded from the majority of family decision-making (McInnis, 1991). Walker- 
Moffat (1995) related that “a woman's most important role in the Mong/Hmong culture is to form alliances between clans through marriage and to provide her husband with many children. Her identity is based on her role as a wife and a mother" (p. 34). Filial piety, a virtue based on respect toward one's family and ancestors, is highly valued within the Mong/Hmong family, and those who go against this traditional practice are considered delinquents (Cerhan, 1990; Walker-Moffat, 1995).

\section{Intergenerational Family Conflict}

According to Cheng et al. (2010), multiple research studies have linked demographic characteristics and psychopathology to suicidal behaviors. However, it is also likely that suicidal behaviors can be influenced by interpersonal relationships, such as family and community cultural norms and values. Although family conflict may affect suicidal behavior regardless of ethnic or cultural background, the impact of family conflict on suicide among immigrants, in particular Asians, may be greater due to family loyalty and sensitivity to shame associated with "loss of face;" a sign of self-blame and embarrassment due to one's failure to meet the expectation of family or others (Zane \& Mak, 2003). For these particular reasons, it is not surprising that Asian American students are more likely to internalize their emotions and have higher rates of parasuicidal behavior than Caucasian students (Cheng et al., 2010; Kisch, Leino, \& Silvermann, 2005; Muehlenkamp, Gutierrez, Osman, \& Barrios, 2005).

Researchers have found that intergenerational family conflict leads to high levels of depression and delinquent behaviors (Aldwin and Greenberger, 1987; Hall, 1987; Su et al., 2005). However, several other researchers have reported that among Asian 
Americans, intergenerational family conflict is a major contributor to parasuicidal behavior and the completion of suicide, even when controlling for depression (Cheng et al., 2010; Lo, 2009; Lorenzo, Pakiz, Reinherz, \& Frost, 1995). For example, the most recent research study on suicide among Asian American college students reported that suicide ideation and attempts may be influenced by not meeting one's family's academic expectations (Cheng et al., 2010).

Lau, Jernewall, Zane, and Myers (2002) conducted a study to identify correlates of suicidal behaviors in a sample of 285 Asian Americans and found that youth who reported high parent-child conflict and were less acculturated were at greater risk of suicidality as these youth faced daily stressors resulting from discrimination from the larger community and cultural conflict arising from their immediate families. Evidently, immigrant families are prone to higher rates of intergenerational family conflict as a result of members being in different stages in the acculturation process within the host country (Sluzki, 1979) Intergenerational family conflict arises in various forms, dependent upon the cultural group and acculturation stage (Su et al., 2005). K. Yang (2003) conducted a qualitative research study that examined felt needs, problems, and community development within Mong/Hmong students and elders and identified intergenerational differences in acculturation as a leading post-immigration family conflict issue among the parent and child relationship. The study found that Mong/Hmong students who perceived themselves to be more acculturated in this country exhibited higher family conflict (K. Yang, 2003). In most post-immigration processes, parents and elders are less likely to relinquish their cultural and traditional values and 
adopt the norms and values of the host culture when compared to their children (Miller \& Lee, 2009; Sluzki, 1979; Su et al., 2005).

According to Rosenthal, Ranier, and Klimidis (1996), among Asian American families, intergenerational conflict intensifies when family roles are reversed—children assume the parental roles while the parents become dependent upon the children, which is quite common within first generation Mong/Hmong families. Vangseng's (1995) qualitative study found that as Mong/Hmong children become fluent in the English language, their non-English speaking parents rely on them to interpret during medical appointments and school visits. They are also given the responsibility to manage the household budget. As Mong/Hmong children become more acculturated into the U.S., they relinquish their cultural values and language, thus becoming more distant from their ethnic culture and contradicting their parents' teachings (Vangseng, 1995).

Traditional Mong/Hmong parents and elders are more committed to maintaining and preserving their cultural identity, which can lead to parent-child conflict as well as a significant rise in mental health issues (Lo, 2009; Su et al., 2005; K. Yang, 2003). As previously indicated, in late 1998 to 2001, a series of Mong/Hmong youth suicides occurred in a California community and made national headlines (Huang, 2006). These suicides prompted the Office of Refugee Resettlement and the Substance Abuse and Mental Health Services Administration (SAMHSA) to conduct a preliminary assessment on this cultural phenomenon. The assessment consisted of focus groups and individual interviews with the victims' families and community members regarding plausible contributing factors to these suicides. The outcome of this assessment identified 
intergenerational family conflict as a primary factor in Mong/Hmong youth mental health deterioration (Southeast Asia Resource Action Center, 2003; DHHS, 2002). Although the findings were disturbing, there remains no follow-up research on the psychological needs of Mong/Hmong youth.

The degree to which intergenerational family conflict predicts suicide risk among Mong/Hmong families has not been thoroughly researched. However, the existing literature suggests that ethnic identity and family members' acculturation stages significantly influence intergenerational family conflict. Therefore, intergenerational family conflict may serve as a significant factor in predicting depression and suicide risk among Mong/Hmong children. Overall, the majority of literature on adolescents and youth suicide reported that depression is the most commonly known factor associated with suicide (Cheng et al., 2010; Gutierrez, Osman, Kopper, Barrios, \& Bagge, 2000; Osman et al., 2001).

\section{Ethnic Identity}

According to Erikson (1968), identity formation is a pivotal task for adolescents. One of the greatest developmental stages of life for an individual to achieve is to successfully identify a personal identity and sense of belonging in society (Erikson, 1950). Individuals who successfully develop a sense of self and personal identity have greater opportunities to maintain positive psychological psychological and behavioral problems. It is through the identity formation phase that individuals develop their own self-identity, separate from their family of origin, and begin to function as independent members of a wider society. Erikson (1950) argued that the formation of one's self- 
identity is developed in a social context; negative views of the dominant society might create negative outcomes, such as negative self-identity or self-hatred. One limitation of this perspective is that Erikson is a developmental theorist whose views do not account for variation in ethnic identity or culture in the articulation of adolescent development. To counter Erikson's theory, Marcia $(1966,1980)$ argued that personal identity is influenced by individual choices (e.g., ego identity) rather than the societal context. In order to further explore how ethnic identity relates to suicidal behavior, this researcher positioned the current study with Erikson's theory_-personal identity is developed in a social context.

According to Erikson (1950) and Durkheim (1951/1987), adolescents and young adults search for and develop their own personal identities in order to feel a sense of belonging in society. This developmental stage is extremely challenging as individuals attempt to position themselves in society. Depending on the individual's racial and cultural backgrounds as well as his or her community, this developmental phase can be overwhelming. For instance, Phinney (2003), a cross-cultural psychologist who has contributed much research to the field of ethnic identity, suggested that minority youth in today's era face the challenges of achieving a personal ethnic identity due to an imbalance of identifying with their ethnic identity and western culture. These minority youth are subject to discrimination, possess less authority in society as compared to White European American youth, and may form and hold values that differ from the greater community. 
Phinney (1990) further suggested that the development of ethnic identity is a complex process in which individuals explore, formulate, and make sense of their membership within an ethnic group in order to secure their identity within the larger society (Phinney, 1990). It is the discovery and connections to one's ethnic community that either hinder or positively contribute to one's identity and behavioral outcomes (Eldering, 1995). Research has produced mixed results with regard to the relationship between ethnic identity and immigrant problem behaviors (inclusive of suicide; Cheng et al., 2010; Kvernmo, \& Heyerdahl, 2003; Marcell, 1994; Shrake \& Rhee, 2004; Tsai, Ying, \& Lee, 2001). Shrake and Rhee (2004) conducted a cross-sectional study that examined three dimensions of ethnic identity (i.e., level of ethnic identity, attitude toward other groups, and perceived discrimination) as predictors of problem behaviors with 217 Korean American adolescents. Results suggested that adolescents who reported a sense of bicultural identity were more likely to integrate and adjust to their ethnic and mainstream communities; thus experiencing less family tension and problem behaviors (Shrake \& Rhee, 2004). Additionally, Tsai et al. (2001) conducted a cross-sectional study that examined how specific domains of cultural orientation, such as language, social affiliation, and cultural pride, related to self-esteem among 353 Chinese college students. Results suggested that cultural orientation significantly impacted self-esteem when compared to age, gender, academic grade point average, and socioeconomic status (Tsai et al., 2001).

The National Latino and Asian American Study (NLAAS; Cheng et al., 2010) provided the most comprehensive examination into the role of culturally relevant 
variables such as family conflict, perceived discrimination, and ethnic identity on suicide ideation and suicide attempts among 2,095 Asian Americans throughout the United States. This cross-sectional study provided the most recent data on suicide risk among Asian Americans. Although the ethnicities of the 2,095 Asian American participants were not reported, data suggested that Asian Americans who reported higher rates of identification with ethnic identity had lower rates of suicide attempts; thus, ethnic identity may serve as a buffer against suicide risk due to the ability to identify a sense of belonging to a group. According to Phinney (1990) and Roberts et al. (1999), group identity is an important factor in defining one's personal and ethnic identity as they relate to a sense of belonging and commitment to a larger group of individuals.

There is limited evidence on how ethnic identity influences suicide among the various ethnic groups within the heterogeneous population of Asian Americans. It is estimated that there are over 60 Asian ethnic groups in this country, and Southeast Asian subgroups are among those living in poverty with increasingly high rates of mental health illnesses and low academic achievement (Cerhan, 1990; Harachi, Catalano, Kim, \& Choi, 2001; Su et al., 2005). Due to these problems and their immigration history, including their continued expansion and acculturation in U.S. society, more research is needed on the impact of ethnic identity development on the mental health of Mong/Hmong youth. Given the existing research studies on ethnic identity and its correlation with behavioral outcomes using Asian Americans and other ethnic groups, it was imperative to examine whether the degree of ethnic identity predicted depression and suicide risk among Mong/Hmong youth. 


\section{Spirituality}

There is reasonable evidence indicating that spirituality is a protective factor against suicidality, as spirituality provides meanings, values, and beliefs (Chandy, Blum, \& Resnick, 1996; Oquendo et al., 2005; Tubergen, Grotenhuis, \& Ultee, 2005). Chandy et al. (1996) conducted a cross-sectional study among 2,681 sexually abused female adolescents to determine behavioral risk outcomes and protective factors. The results suggested that individuals who reported involvement in spiritual activities were at a lower risk of suicide, indicating religion to be a protective factor against suicidality. In another study, Garroutte et al. (2003) conducted a cross-sectional study among 1,465 Native Americans in the Northern Plains during 1997 and 1999 to examine the relationship of spirituality to the lifetime prevalence of suicide attempts. The study found that that degree of religiosity and involvement in religious activities presented lower prevalence of suicide risk than those who reported minimal to no involvement in spiritual events, suggesting that positive cultural identity may be a protective factor against suicidality among Native Americans. Although the majority of the research was conducted among Catholics, Protestants, and Christians, it was of interest to this researcher to explore how traditional spirituality and healing predicted suicide risk among Mong/Hmong youth. Due to the uniqueness of the traditional healing belief system and the spiritual interpretation of death, it was imperative to explore how traditional beliefs influenced suicide risk among Mong/Hmong youth.

Traditional Mong/Hmong culture adhere to a belief system that personal and social issues are intertwined with the traditional religious belief of Shamanism and 
Animism. Shamanism involves the participation of a shaman/shawoman who spiritually goes into a trance and reaches an altered state of consciousness in order to interact with the spiritual world (Fadiman, 1997). Animism is the traditional spiritual belief in which all natural objects (e.g., rocks, water, sand) are living beings and all living beings and creatures are interconnected. Due to the Mong/Hmong's contact with French Missionaries in the 1950s and the displacement of Mong/Hmong refugees since 1975, it is believed that half of all Mong/Hmong individuals in the United States converted to Christianity or another form of religious orientation. The other half is believed to have continued the traditional and religious practice of Shamanism/Animism (The Cultural Orientation Project, 2004).

Traditionally, the Mong/Hmong believe that diseases, misfortunes, mental illnesses, physical disabilities, and death are caused by the deceased who were given improper traditional funeral rite of passage into the after world or evil spirits (Bliatout, 2003; Mouanoutoua, 2003). According to Quincy (1995), evil spirits are invisible and live among human beings, eager to consume their souls. It is believed that within each person there lies three or more souls. When souls wander off or are kidnapped by evil spirits, sickness and mental illness occur. Wandering souls are taken very seriously as a wandering soul may eventually lead to death. To deter sickness and suicidal ideation/attempt from getting worse, herbal medicines are utilized. However, when sickness cannot be cured by herbal medicines, a Shaman/Shawoman is called to perform a healing ceremony to retrieve and guide the lost souls to their original owners. It is believed that once the lost souls are reunited with their owners, the individual's sickness 
will either improve or be cured. During the healing ceremony, an animal may be sacrificed as the Shaman/Shawoman spiritually leaves his or her body, enters a trance into the spiritual world, and spiritually utilizes the sacrificed animal to negotiate the return of the ill person's missing souls from the ancestors or evil spirits. If the Shaman/Shawoman cannot retrieve the missing souls, it is likely that the individual will die and be reincarnated into another human being, animal, or natural object (The Cultural Orientation Project, 2004; Fadiman, 1997; Siegal \& McSilver, 2001).

Among traditional Mong/Hmong community members, suicidal individuals are thought to have lost their souls to evil spirits or the deceased. Rates, risks, and protective factors for suicide vary across religions; therefore, given the influence of various religious beliefs and stages of acculturation, it was of unique interest to this researcher to understand the role of the traditional Mong/Hmong belief of spirituality/healing practices and its relationship among the variables of intergenerational family conflict, ethnic identity, and acculturation stage, as well as its impact on depression and suicide risk.

\section{Depression}

Depression is a psychological mental health disorder that is highly associated with suicidality. According to the Diagnostic and Statistical Manual of Mental Disorders (4th ed., text rev.; DSM-IV-TR; American Psychiatric Association, 2000), "Up to 15\% of individuals with severe major depressive disorder die by suicide" (p. 371). When examining suicide risk factors it is important to take depression into account as previous studies have indicated this psychological mental health disorder to be a primary risk factor in suicidality among various groups (e.g., age, gender, race, socioeconomic status; 
Eisenberg, Gollust, Golberstein, \& Hefner, 2007; Gencoz \& Or, 2006; Gutierrez et al., 2000). For example, in Eisenberg et al.'s (2007) web-based cross-sectional study, researchers examined the prevalence and correlation of depression, anxiety, and suicidality among 2,843 university students and found a significant relationship between suicidal thoughts and depression.

Similar to the conceptualization of suicidality among traditional Mong/Hmong, depression has its own unique definition. As mentioned in the DSM-IV-TR (2000), culture has a tremendous impact on the experience and communication of symptoms of depression. In the western culture, depression is expressed in terms of emotion, such as feeling worthless and hopeless. In most Asian cultures, the experience of depression is conveyed through somatic terms, such as feeling weak and imbalanced (DSM-IV-TR, 2000). Within the Mong/Hmong culture, Bliatout (2003) stated that extreme emotional imbalance, such as depression, is caused by a "loss of soul" in which the individual's unhappiness has resulted in either the soul leaving the physical body or being captured by a spirit. When the Mong/Hmong terminology of depression is translated into English, it means "difficult" or "broken liver" problem. The liver is referred to as the broken organ rather than the heart because the Mong/Hmong believe that the liver is the organ where emotions reside while in the western culture emotional feelings are expressed by using the word "heart" (e.g., broken hearted, open hearted, light hearted; Bliatout, 2003; Mouanoutoua, 2003).

It has been well documented that first generation Southeast Asian refugees suffer from high rates of depression and posttraumatic stress disorder (Bliatout, 2003; Miller \& 
Lee, 2009; Mouanoutoua, 2003; Nicholson, 1997; Su et al., 2005; Ying \& Akursu, 1997). For instance, Bliatout (2003) related that on an emotional level, Mong/Hmong parents feel a great sense of loss of who they are as they struggle to qualify for menial jobs and maintain control over their families. Depression has been highly linked to first generation Mong/Hmong adults in the United States, because as they transition from one culture to another they undergo individual and familial changes. Although most studies on this population were conducted with adults, parental depression has been associated to their children's psychological well-being (Beardslee, Gladstone, Wright, \& Cooper, 2003; Riley \& Broitman, 2003). Because of this association, researchers and clinical providers have suggested that children whose parents are psychologically imbalanced are at a higher risk for emotional disorders and family conflict (Beardslee et al., 2003; Riley \& Broitman, 2003).

Many Mong/Hmong communities in the United States continue to practice healing ceremonies to cure individuals from sickness and mental illness. Utilizing western medicine and mental health treatment services has been a challenge for the Mong/Hmong. For example, Cerhan (1990) stated, "In seeking help for themselves [Mong/Hmong], clan members are the first source that Mong/Hmong turn to for assistance with an adjustment or mental health problem" (p. 69), as self-disclosure of a mental health problem is seen as a form of weakness within the individual and family; therefore, mental illness is not acknowledged within the Mong/Hmong culture. Furthermore, translation of psychiatric mental health diagnoses from English to the Mong/Hmong language is a barrier in that the Mong/Hmong do not have mental health 
diagnose terms such as schizophrenia, psychosis, and bipolar disorders. For instance, when translated into the Mong/Hmong language, the majority of western mental health diagnoses (mental illnesses) simply mean "crazy;" as such, individuals who seek mental health services are stigmatized as incompetent within the Mong/Hmong community (Cerhan, 1990; Mouanoutoua, 2003; Mouanoutoua \& Brown, 1995; Nicholson, 1997; Yee, n.d.).

Chen, Guarnaccia, and Chung (2003) utilized the Center for Epidemiological Studies Depression Scale (Radloff, 1977) to examine the relationship between acculturation and depression among Chinese and Japanese Americans $(n=102)$, and found that higher acculturation was associated with greater affective symptoms and fewer somatic symptoms. In Foss' (2001) qualitative study on acculturation and depression among Vietnamese and Mong/Hmong Americans $(n=30)$, findings indicated that less acculturation was associated with greater anxiety and depression.

Although there have been numerous studies on Asian Americans and psychological distress, research on depression and its linkage to suicide risk among Mong/Hmong youth is limited. Therefore, understanding the prevalence, severity, and relationship of depression to other factors is needed in order to determine whether depression predicts suicidality among Mong/Hmong youth. In sum, given the existing literature and the following conceptual framework to explain suicidal behaviors among Mong/Hmong youth, the current study was unique in that it examined a multitude of psycho-sociocultural dimensions (i.e., intergenerational family conflict, ethnic identity, 
spirituality, depression, acculturation) and their relationships with each other in addition to predicting how they influenced depression and suicide risk.

\section{Conceptual Framework}

The phenomenon of Mong/Hmong youth suicide attempts and suicide completion has not been fully studied. Although there are a variety of explanatory theories regarding suicidal behaviors (e.g., anomie theory, biological/genetic theories, social learning theory), in the western culture there remains no empirically tested theory explaining the etiology of this issue among Southeast Asians, particularly Mong/Hmong youth. However, researchers such as I. Chung (2004), the Committee on Cultural Psychiatry, Group for the Advancement of Psychiatry (CCPGAP, 1989), and Tatman (2004) have concluded that acculturation theory is relevant to explaining the etiology of Southeast Asian American youth emotional and behavioral problems. Additionally, anomie theory (Durkheim, 1951/1987) provides a conceptual basis for understanding external factors that contribute to immigrant youth suicidal behavior within the western culture.

Therefore, acculturation theory and anomie theory are examined to help explain the social problem of Mong/Hmong youth suicide.

\section{Acculturation Theory}

Acculturation theory has been applied to explain various behavioral outcomes of immigrants in this country. Initially, anthropologists developed the concept to study group-level phenomena; nowadays it is frequently utilized to study individual-level psychological phenomena (CCPGAP, 1989). For the purpose of the current study and as indicated by the CCPGAP (1989), dominant individual or cultural group/society refers to 
the majority of individuals who share similar cultural and worldviews, and less dominant individuals or groups refers to individuals, in particular immigrants or ethnic minorities, who possess less power and representation within mainstream society.

In examining the formation of the acculturation process among ethnic minorities, Berry (1993) argued that one cannot examine acculturation alone without examining how ethnic identity impacts the acculturation process of immigrant psychological functioning in the United States. Because of Berry's interest in examining how psycho-sociocultural factors influence suicide risk among immigrant youth and his involvement in developing the acculturation theoretical framework utilized to explain how the acculturation process influences suicide risk among ethnic minorities, this writer chose to utilize the acculturation theoretical framework from the CCPGAP (1989) to explain how acculturation impacts suicide risk among Mong/Hmong youth. The acculturation theory as developed by the CCPGAP is based on the work of several leading psychiatrists and psychologists who were involved in developing a rigorous national report on suicide among major racial and ethnic groups in the United States (CCPGAP, 1989).

The primary focus of acculturation theory explains how individuals, in particular less dominant individuals, are culturally affected when transitioning from one culture to another as they adjust into the host (i.e., dominant) culture's worldview (Farver, Xu, Bhadha, Narang, \& Lieber, 2007). Acculturation theory assumes that less dominant individuals who come in contact with the dominant cultural group or society will eventually alter their cultural values, beliefs, and behaviors as a result of continuous firsthand contact with the new dominant culture. 
The theory emphasizes the process of acculturation as a stressful experience of ecological, cultural, interpersonal, and psychological changes while transitioning from one culture to another. Because of the stress associated with the acculturation process, the CCPGAP (1989), coined the term acculturative stress, which "implies that persistent intercultural contact brings stressors to bear on the less dominant group, inducing a state of tension in the group and anxiety in its component individuals" (p. 115). Historically, psychologists believed that the tension and anxiety experienced by the less dominant individual or group eventually led to mental health issues among those involved. However, studies eventually proved that the outcome of acculturation depended upon several interacting factors, such the "phases of acculturation, the mode of acculturation, the type of acculturating group, the nature of the more dominant cultural group, the sociocultural characteristics of the less dominant group, and the psychological characteristics of the acculturating individuals" (CCPGAP, 1989, p. 116). As a result, the following is a summary of the acculturation theory as applied to the Mong/Hmong.

The acculturation process includes five distinct phases experienced by the less dominant individual (i.e., Mong/Hmong): (a) pre-contact phase, (b) contact phase, (c) conflict phase, (d) crisis phase, and (e) adaptation. The pre-contact phase occurs when two independent individuals (i.e., Mong/Hmong and non-Mong/Hmong) exist and value different sets of cultural characteristics such as language, religious beliefs, childrearing practices, family, and education. Following the pre-contact phase is the initial contact phase in which the two independent individuals begin to interact within the community. This phase usually occurs when different ethnic individuals begin to socialize within the 
educational and community settings. This interaction results in the dominant individual and society's influence on the less dominant individual to change and conform to the behaviors and culture of the dominant society. It is at this phase that the less dominant individual begins to contemplate his or her identity within the larger community. Following the contact phase is the conflict phase, in which the less dominant individual experiences tension and pressure from the dominant individual to change their cultural beliefs and ways of life. When conflict and tension begin to surface between the two individuals, crisis occurs. It is at this crisis phase that some form of resolution is required. Although a solution may not be attainable to decrease the tension between the two individuals, an adaptation phase may occur in order to stabilize the intergroup relations (CCPGAP, 1989).

According to the CCPGAP (1989), the conflict and crisis phases present social and psychological issues for the less dominant individual. When applying the acculturation phase to Mong/Hmong youth, this writer postulates that when Mong/Hmong youth relocates to the United States from Thailand, they are forced to adapt to the western educational system, thus conforming to the norms of their peers, teachers, and community. As the youth debates whether they will conform to the norms, the youth is also challenged in negotiating whether to maintain their ethnic and cultural identity. This process leads directly to one of the four approaches of acculturation: (a) assimilate, (b) integrate, (c) separate, or (d) marginalize (Berry, 1993; CCPGAP, 1989). The CCPGAP (1989) indicated that in the assimilation approach, the less dominant individual eventually relinquishes their ethnic and cultural values and adopts 
the cultural characteristics and values of the dominant society. In integration, the individual retains their cultural values while integrating into the larger social system. This approach helps the individual find a balance in maintaining both the ethnic and host cultures. In the separation level, the individual withdraws from the dominant society and lives independently to preserve their cultural identity. Lastly, marginalization occurs when the individual has lost virtually all their cultural identity and origin without replacing them or participating in the dominant society. It is during this mode of acculturation that individuals are displaced by feeling hopeless and alienated and experience identity confusion, which may result in defiant and suicidal behaviors (CCPGAP, 1989).

Berry (1993) proposed that the acculturation time frame of individuals varies depending on the internal cohesiveness of the less dominant society, the nature and intensity of contact between the two independent individuals, and the degree to which the less dominant individual resists or accepts change. For these various factors, he identified four paths in which immigrants, or less dominant individuals, associate with the dominant culture based on the decision to eventually assimilate, integrate, separate, or marginalize (see Figure 1). 


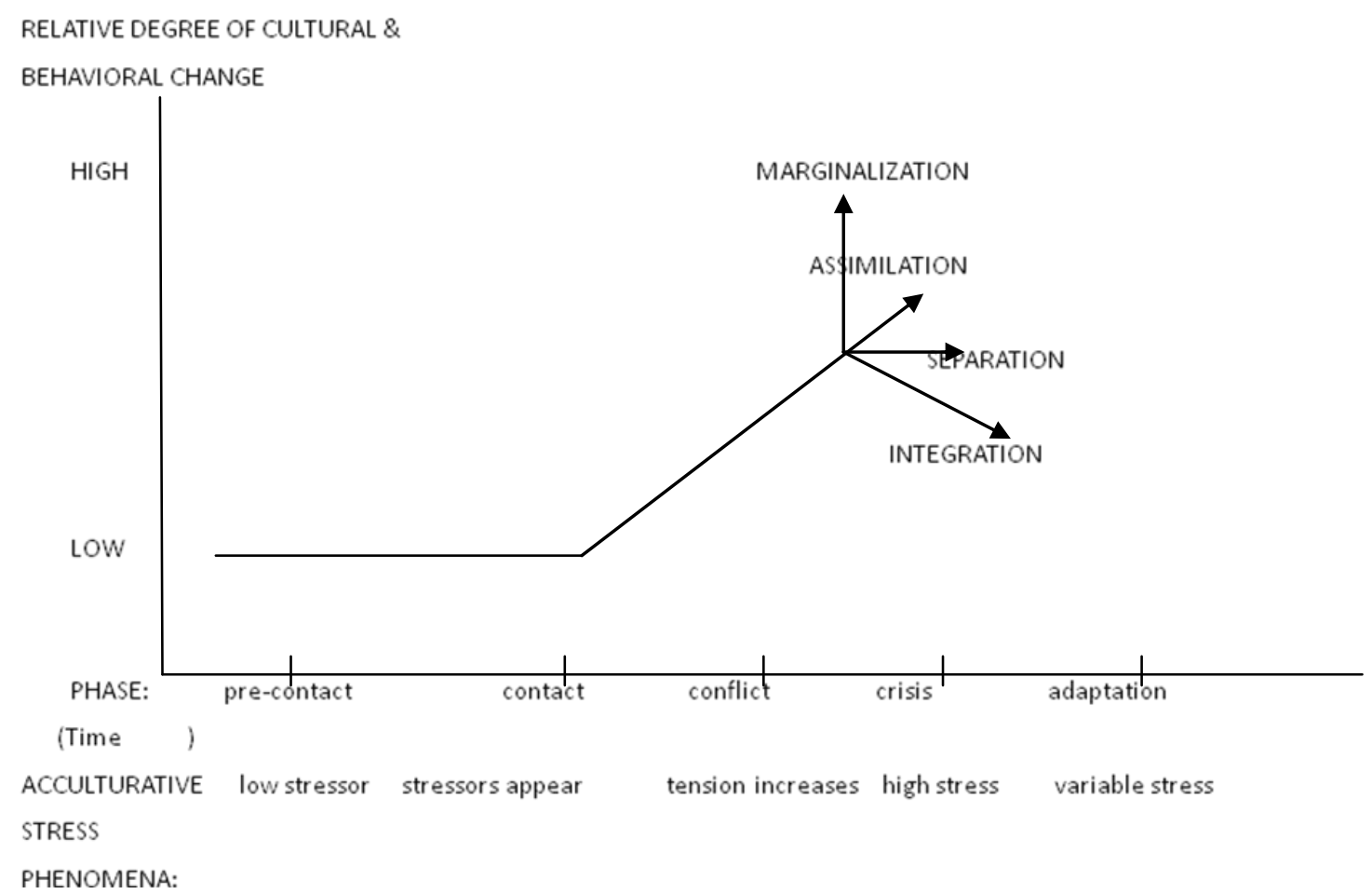

Figure 1. Variations in acculturative stress as a function of phase and mode of acculturation (CCPGAP, 1989).

Although these four paths reflect the acculturation process, they may not hold true for those who have acculturated or are in the process of acculturating into the dominant society.

According to the CCPGAP (1989), acculturation has been widely misunderstood to be a linear phenomenon in which less dominant individuals eventually relinquish their cultural characteristics, such as their customs, beliefs, and behaviors, and assume the cultural characteristics of the dominant society. However, the "tendency for this to occur is greatest in societies where there is an assimilationist or culturally homogeneous ideology, but it is not inevitable" (CCPGAP, 1989, p. 117). The degree to which acculturation is attained is greatly influenced by both the nature of the less dominant individual and by the degree to which the dominant society accepts or rejects the less 
dominant individual. According to the CCPGAP, these two issues can be summed by asking the following questions that are intended to lead the less dominant individual to an acculturation approach (i.e., assimilation, integration, separation, or marginalization): (a) Is my cultural identity of value and to be retained?, and (b) Are positive relations with the larger, dominant society to be sought?

The stress associated with the acculturation process has been correlated with severe mental health issues (CCPGAP, 1989). According to the CCPGAP (1989) and Sue (2004), acculturation theory provides the best explanation for understanding the emotional and behavioral health of immigrant children. For instance, the CCPGAP stated that when immigrant youth are unable to feel a sense of belonging to either their ethnic or mainstream communities, they experience identity crisis, role confusion, and cultural and behavioral changes, such as increased substance abuse, intergenerational family conflict, and social and personal violence. For these reasons, it was imperative for the current study to take into account the role of acculturation and its association with ethnic identity, intergenerational family conflict, depression, and spirituality on suicide risk among Mong/Hmong youth.

\section{Anomic/Anomie Theory}

Durkheim (1951/1987), a sociologist and founding father of the anomic/anomie theory, proposed that suicide is linked to society and the social environment (i.e., family, political society, and occupational groups). The anomic/anomie theory posits that there are four types of suicide based on the degree of imbalance of two social forces: social integration and moral regulation. Integration refers to the extent to which individuals 
socialize with a person or group and regulation refers to the normative or moral demands placed on the individual that come with membership in a group (e.g., values and beliefs). The four types of suicide are: (a) egoistic, (b) altruistic, (c) anomic/anomie, and (d) fatalistic.

Egoistic suicide occurs when individuals lack integration to society; thus, they are left with minimal social support and guidance that predisposes them to higher suicide rates. Altruistic suicide is the result of too much integration when individuals sacrifice themselves for others (e.g., religious commandments and more common among military members). Anomic/anomie suicide is the result of an imbalance of means and needs; it occurs as a result of the lack of regulation of the individual by society. According to Durkheim (1951/1987), individual needs and satisfaction were assumed to be regulated by society; therefore, when the regulation is eradicated, fatalistic suicide occurs. Individuals in this state are assumed to have poor coping skills, minimal insight, and may not have possible solutions to improve their life; thus, when in a state of misery, the individual turns to fatalistic suicide (Durkheim, 1951/1987).

For the purpose of the current study, anomie theory was used to explain the social problem of suicide among Mong/Hmong youth in the United States Although anomie theory does not specifically look at suicide attempts and suicide among cultural groups, it does take into account how individuals in new settings are impacted by society when given few norms (e.g., societal rules/regulations and directions) to follow, resulting in individuals committing a wide range of destructive acts, including killing themselves (Durkheim, 1951/1987). Furthermore, due to the lack of empirical research and 
explanatory theories on the etiology of Mong/Hmong youth suicide, it was this researcher's opinion that the anomie theory could provide sufficient explanation for the cause of this phenomenon.

The basic assumption of anomie theory is that no individual can live happily unless needs are sufficiently proportional to means. When needs are not met, this leads to dissatisfaction with life. The individual looks to social systems (e.g., religious affiliation, family, government) to help guide them in meeting their needs (Durkheim, 1951/1987). According to Bearman (1991), anomie theory suggests that individuals cannot function or find their place in society without clear rules and guidance. When an individual is given insufficient guidance and unregulated information from the social structure of society, the individual is left without direction to overcome obstacles. Therefore, suicide is a result of the instability of social norms and institutional disorganization (e.g., values and beliefs of religious groups or larger society differ from those of the individual; Dhossche, 2003). Although an individual act, Durkheim (1951/1987) argued that suicide is a product of the social structure of any given society.

Hassan (1998) stated that when there is an absence of regulation and high integration in the dominant society, anomie suicide occurs. For example, when Mong/Hmong youth begin to integrate into the dominant society, many face sudden expectations and challenges from the dominant society in which they feel they must disown their cultural identity and adopt western values (Gibbs \& Huang, 1998). Durkheim (1951/1987) argued that when individuals are in a state of anomie (i.e., high integration with low regulation from the social structure of society), they experience a 
temporary rapid social change; a result of a crisis interrupting their social life. Without sufficient support from the social structure of society, these crises may result in deviant acts by the individual (e.g., crimes, substance abuse, suicide). For instance, when the Mong/Hmong immigrated to the United States, their traditional healing practices were disrupted as the dominant culture devalued the traditional healing practice of sacrificing animals to the spiritual world (Fadiman, 1997). This rapid social change became distressful for some of the Mong/Hmong as they attempted to adjust to the new setting (i.e., country). For some of these Mong/Hmong communities, they lost a sense of authoritative normative regulation, thus leading to individuals experiencing cultural conflict and psychological issues.

Without given proper guidance from the larger society to function within this country, many Mong/Hmong have struggled with balancing their own cultural norms (e.g., family and religious practices) with the values and beliefs of the western culture (e.g., individualism, non-Animism religious practices). According to Jesilow \& Xiong (2007), the Mong/Hmong culture functions as a collective group which emphasizes cohesiveness, inclusive of family and the community. Whereas, the American culture emphasizes on individualism; focusing on one self. As a result, these personal and familial challenges such as acculturative stress, intergenerational family conflict, severe mental health illnesses, and incidences of suicide attempts and completion of suicide have impacted the Mong/Hmong community (The California Endowment, 2006; Huang et al., 2004; Jesilow \& Xiong, 2007). 


\section{Limitations of Theory}

A major weakness of the acculturation theory is the lack of consistency in the conceptualization of acculturation. Depending upon which model researchers choose, acculturation theory can be explained from a bi-dimensional or multidimensional perspective. Theories of acculturation have evolved through the years as researchers and theorists have argued whether acculturation is bi-dimensional or multidimensional (Berry, 1993). For instance, some scholars suggest that acculturation is bi-dimensional as immigrant individuals can maintain their cultural values while adapting to the host culture (Kim \& Abreu, 2001; Ryder, Alden, \& Paulhus, 2000). R. H. Chung, Kim, and Abreu (2004) argued that acculturation is uni-dimensional and occurs continuously in which acquisition of the host cultural values is accompanied by loss of culture-of-origin. After an extensive literature review, the majority of research provided strong evidence that acculturation is bi-dimensional (R. H. Chung et al., 2004; Kim \& Abreu, 2001; Ryder et al., 2000). Although there are existing studies in which researchers have postulated that acculturation is multidimensional (i.e., acculturation is a process involving languages, beliefs, values, and structural assimilation), results indicated a bi-dimensional score (Barrett, Sonderegger, \& Sonderegger, 2002; Becker, Burwell, Navara, \& Gilman, 2003; Elder, Broyles, Brennan, Zuniga de Nuncio, \& Nader, 2005; Gonzalez \& Shriver, 2004). According to Zane and Mak (2003), there is a discrepancy in acculturation and mental health outcomes due to the fact that acculturation has not been operationalized consistently across studies, leading to mixed results. For instance, some acculturation measurements are designed specifically for particular ethnic groups (De Leon \& Mendez, 
1996; Snowden \& Hines, 1999), while others are designed for the general immigrant population (Stephenson, 2000).

Furthermore, acculturation theory assumes that all immigrants undergo similar psychological and cultural experiences in the host country. Regardless of which model is applied to acculturation research and depending on the variables of interest, acculturation theory can be applied at a micro or macro level. For this particular research study, the acculturation theory was applied at a micro level as it is the individual who determines his or her level of acculturation despite the influence of the dominant society. Additionally, the general framework of acculturation theory outlines and links ethnic culture and psychological factors as vital components of the acculturation process. Although the anomie theory is frequently used to explain the causation of suicidality, the theory fails to take into account the potential impacts of race and ethnicity on suicide risk from the macro level. Therefore, it was of interest to this researcher to further explore the influence of both the family and spiritual systems, coupled with ethnic identity, depression, and acculturation, to determine their impact on suicide risk among Mong/Hmong youth. 


\section{Chapter 3: Research Methodology Research Questions and Hypotheses}

The following independent variables were examined with regard to their influence on suicide risk (dependent variable) among Mong/Hmong youth: intergenerational family conflict, ethnic identity, acculturation, depression, and spirituality. Due to strong evidence of depression being a predictor of suicide risk among the general population, depression was also examined as a dependent variable. The research questions and hypotheses that guided this study were (see Appendix J for outline of research questions):

Question 1: What is the rate of suicide ideation and attempts among Mong/Hmong youth within this sampled population?

Hypothesis 1: At least 50\% of the participants will report they have had suicidal ideation and attempts.

Question 2: Is there an association between ethnic identity and depression among Mong/Hmong youth?

Hypothesis 2: Depression will increase with lower levels of ethnic identity. According to previous studies on ethnic identity and psychosocial behavioral outcomes, individuals who report less identification with their ethnic identity are more likely to develop behavioral issues such as depression.

Question 3: Is there a relationship between intergenerational family conflict, traditional and spiritual healing belief system, and ethnic identity among Mong/Hmong youth? 
Hypothesis 3: Intergenerational family conflict will increase with lower levels of ethnic identity and lower spiritual beliefs in Shamanism/Animism. According to current literature on intergenerational family conflict, ethnic identity, and spirituality, as individuals distance themselves from their family ethnic identity and spiritual belief system, family conflict increases.

Question 4: How well do acculturation, ethnic identity, and intergenerational family conflict predict depression among Mong/Hmong youth?

Hypothesis 4: High intergenerational family conflict, low ethnic identity, and low acculturation will predict depression among Mong/Hmong youth. Individuals who distance themselves from their family ethnic identity are more likely to experience increased intergenerational family conflict and are more likely to separate from their cultural identity, thus leading to a depressive behavioral outcome.

Question 5: What are the effects of ethnic identity, intergenerational family conflict, depression, family household size, and traditional and spiritual healing beliefs on suicide risk among Mong/Hmong youth?

Hypothesis 5: Low ethnic identity, high intergenerational family conflict, and high depression will predict suicide risk among Mong/Hmong youth. Due to history of suicide risk among ethnic minority youth, individuals with low self-identification of ethnic identity are more likely to report higher intergenerational family conflict, thus leading to increased depression and eventually experiencing suicidal behavior. 


\section{Study Design}

This research was a cross-sectional, exploratory study that used a purposive sampling method in addition to snowball sampling. Study participants were recruited from three California academic institutions: (a) California State University, Chico; (b) California State University, Sacramento; and (c) University of California, Davis. Each of these universities has established Mong/Hmong student organizations, which facilitated participant recruitment. The university setting was chosen due to the accessibility of Mong/Hmong adults between the ages of 18 and 25. Prior to the data collection stage of this research study, the Portland State University, Human Subjects Research Review Committee approved the study.

\section{Sample Population}

The sample population consisted of Mong/Hmong youth between the ages of 18 to 25. The terminology "youth" does not necessary refer to adolescents, but also emerging adults (Arnett, 2000; Erikson, 1968; Juang, Nguyen, \& Lin, 2006; Kang, Okazaki, Abelmann, Kim-Prieto, \& Lan, 2010).

\section{Participant Recruitment}

According to Hyman, Vu, and Beiser (2000), Messer and Rasmussen (1986), and Um (2003), although the Southeast Asian community faces high poverty rates and continues to struggle with raising their children in this country, they value the importance of education and strongly encourage their children to become academically successful. With high expectations from their parents, Southeast Asian students are burdened with the academic challenges and family expectations of earning good grades, attaining a 
higher education, and graduating from college. Um stated that these students face numerous challenges in their education and lack the necessary support services to deal with their emotional behavior (e.g., anxiety, depression, suicidal thoughts). As a result, the recruitment of participants in a tertiary educational setting was beneficial to this research study as the data were used to validate whether cultural factors contributed to suicide risk among Mong/Hmong youth.

Although the identity development literature supports the value of including younger participants, cultural consideration determined that the recruitment of minors would pose a significant challenge. According to Ellis (2002), within the Mong/Hmong community, suicide is considered a taboo topic of discussion and is dealt with among the immediate family as they believe that such psychological thinking and action is caused by spirituality. Therefore, if minors were to be recruited for this study, it would likely have been extremely difficult to obtain consent from their parents.

The purposive sampling method was used to recruit participants in the university setting. This researcher recruited 165 participants in order to generalize to Mong/Hmong college youth between the ages of 18 to 25 in northern California. The selection process of the participants was based upon a non-probability sampling method. Snowball sampling technique was utilized to recruit additional participants in order to conduct the analyses. Snowball sampling is a technique in which surveyed participants assist by locating or referring other participants to the study. Although snowball sampling is largely utilized in qualitative studies, Rubin and Babbie (2001) stated that snowball 
sampling is appropriate for research studies that aim to investigate special populations that are difficult to locate.

This researcher made successful contact with each of the identified universities' Mong/Hmong student organizations' elected officials to discuss the research study and its effects on the university campus and community (e.g., bring mental health awareness to the Mong/Hmong student body and community, provide additional student support services for Mong/Hmong students, etc.). This researcher asked permission to present the research study at an organization meeting to recruit research participants, and the responses were overwhelmingly supportive.

\section{Inclusion and Exclusion Criteria}

The eligible criteria were to: (a) identify themselves as being of the Mong/Hmong ethnic cultural background, (b) be between the ages of 18 and 25, and (c) attend one of the participating academic institutions. Additionally, individuals who appeared to this researcher to be actively psychotic or emotionally distressed were excluded from the study (the PI conducting this research study is a CA Associate Clinical Social Worker). All participants $(N=165)$ were informed of the inclusion criteria before receiving the informed consent and instruments; therefore, this limited the process of excluding cases from the study.

\section{Instrumentation}

According to Fugita (as cited in Serafica, Schwebel, Russell, Isaac, \& Myers, 1990), when researchers attempt to use standardized measurements on Asian Americans, they face the challenge of participants misinterpreting questions; that is, Asian Americans 
"respond differently to decontextualized instruments than western subjects" (p. 69). Asians have a greater difficulty responding to abstract questions and are likely to "focus more on the evaluative potential of their responses" (Serafica et al., 1990, p. 69). As a result, none of the measurements used in this research was developed specifically to measure outcomes on the Mong/Hmong population, except for the Mong/Hmong Kev Ntseeg Instrument that was developed by this researcher to measure the traditional and spiritual healing beliefs of the participants. Due to the sensitivity of the research topic, it was imperative that this researcher took caution in selecting clinically sound and culturally appropriate measurements in order to protect and minimize physical and mental harm to the participants.

This study utilized eight self-report questionnaires to determine whether psychosociocultural factors influence suicide risk among Mong/Hmong youth. All documents were printed in the English language because research has indicated that less than $50 \%$ of Mong/Hmong youth are literate in their native language (McGinn, 1989) and the sampled population included university students who spoke English.

\section{Demographic Questionnaire}

A 15-item demographic questionnaire was included in the data collection process (see Appendix B). The questionnaire asked the participants to mark the best answer that described their personal, family, and academic status (e.g., gender, income, and employment). These variables provided specific characteristics of the sample to determine whether certain variables influenced suicide risk among Mong/Hmong youth. 


\section{Asian American Family Conflicts Scale-Original}

The Asian American Family Conflicts Scale-Original (FCS; Lee et al., 2000;

Appendix C) was developed to assess the likelihood and seriousness of family conflicts in values and practices among U.S.-raised children and their immigrant parents. The measurement is based on 10 items with two subscales: (a) FCS-Likelihood (scored on a 5-point scale in which1=never to 5=always) and (b) FCS-Seriousness (scored on a 5point scale in which $1=$ not at all to 5=extremely); a higher score indicates a greater likelihood or seriousness of family conflict as perceived by the participant. The overall family conflict intensity score is obtained by summing the total score of the two subscales, FCS-Likelihood and FCS-Seriousness.

The development of the FCS was tested on three samples of Asian American college students $(n=186, n=153, n=109)$ who ranged between the ages of 16 to 52 years-old. It is worth noting that in the first two samples there was an number of Mong/Hmong respondents (less than 19). Across all three studies, the mean total score of the results were: $31.24(S D=10.10), 31.72(S D=7.66)$, and $27.398(S D=9.44)$. The measurement presented good internal reliability—FCS-Likelihood ranged between .81 and .89 and FCS-Seriousness ranged between .84 and .91 . The stability coefficients for FCS-Likelihood and FCS-Seriousness were .80 and .85 and the inter-scale correlation was $r=74$. According to Lee et al. (2000), the concurrent validity of the FCS was established using the following measurements: (a) the Social, Attitudinal, Familial, Environment Acculturation Stress Scale (SAFE); (b) the Family Adaptation and Cohesion Emotions Scales II; and (c) the Parent and Adolescent Community Scales. 
The FCS has been applied to two research studies on the Mong/Hmong population and has demonstrated good internal reliability with Cronbach's alphas of .94 (Su et al., 2005) and .86 (Miller \& Lee, 2009). The FCS has been correlated with a variety of outcomes measures inclusive of acculturation (Fu, 2002; Huang, 2006; Leong, 2005) and psychological adjustment (Lee et al., 2005).

\section{Multigroup Ethnic Identity Measure}

The Multigroup Ethnic Identity Measure (MEIM; Phinney, 1992; Appendix D) is the most widely used instrument to measure ethnic identity among a multitude of ethnic minorities within this country (Worrell, Conyers, Mpofu, \& Vandiver, 2006). The measurement was originally developed to assess four aspects of ethnic identity: (a) identity achievement, (b) belonging, (c) ethnic behavior, and (d) other group orientation. More recently, the MEIM has been revised to include the measurement of identity achievement of adolescent youth (12 to 19-years-old): (a) ethnic identity search (a developmental and cognitive component) and (b) affirmation, belong, commitment; an affective component; (Robert et al., 1999). The revised MEIM includes 15 items; the first 12 are rated on a 4-point Likert scale from 1 (strongly disagree) to 4 (strongly agree) and the last three pertain to the identification and categorization by ethnicity. Of the first 12 items, five measure ethnic identity search and seven measure affirmation, belonging, and commitment. The mean of the first 12 items provides a global score; however, if desired, the means for ethnic identity search (five items) and affirmation, belonging, and commitment (seven items) can be computed to measure the two factors. Overall, higher scores (i.e., 12 to 48) indicate ethnic identity achievement and lower scores reflect 
identity diffusion or confusion (Roberts et al, 1999). The original MEIM has shown good reliability with Cronbach's alphas of .81 to 89 , across ethnic groups and ages (Phinney, 1992; Roberts et al., 1999). In the revised MEIM, Roberts et al. (1999) validated the instrument's construct validity among an ethnically diverse population of 253 Central American youth; thus, indicating good internal consistency, yielding Cronbach's alpha of .81 , across ethnic youth.

\section{Suinn-Lew Asian Self-Identity Acculturation Scale}

The Suinn-Lew Asian Self-Identity Acculturation Scale (SL-ASIA; Suinn et al., 1992; Appendix E) was developed to assess the cognitive, behavioral, and attitudinal aspects of acculturation among Asian Americans. The SL-ASIA is based on 26 items and scored on a 5-point scale from 1 (low acculturation) to 5 (high acculturation). The acculturation scores are obtained by summing the total score and dividing by 26 .

Suinn et al. (1995) applied the SL-ASIA to 324 Asian American college students and found a Cronbach's alpha of .79. The SL-ASIA is widely utilized in measuring the acculturation levels of Asian Americans and has shown good consistency, Cronbach's alpha of .68 to .88 (Ponterotto, Baluch, \& Carielli, 1998). For the purpose of this study, the SL-ASIA was slightly altered to include the targeted ethnic group, Mong/Hmong. The term "Mong/Hmong" was inserted into the survey in order to provide the participants a sense of ethnic identity. Given the adapted scale, the reliability of this instrument was .67.

\section{Zung Self-Rating Depression Scale}

The Zung Self-Rating Depression Scale (SDS; Zung, 1965; Appendix F) was utilized in this study to measure participants' depression status to determine whether 
depression is linked to suicide risk among Mong/Hmong youth. The SDS is composed of three common clinical diagnostic criteria of depression symptoms: (a) pervasive affect, (b) physiological equivalents, and (c) psychological concomitants. The measurement is based on 20 items and is scored on a 4-point scale from 1 (A little of the time) to 4 (Most of the time). Half of the items are worded as symptomatically positive, and the other half are negative and reverse scored. Once the 10 items are reversed, all 20 items can be summed to obtain a total severity score. The higher the score the greater the level of depression. The highest possible score is 80 .

The SDS has been applied in cross-cultural settings (Marsella, Kinzie, \& Gordon, 1973; Zung, 1965) among the Asian population, inclusive of Mong/Hmong Americans (Westermeyer, n.d.). The reliability of the scale has shown fairly good consistency, Cronbach's alpha of .79. Zung (1965) reported a split-half reliability of 0.73. The SDS has strong correlations with other depression scales, such as the Minnesota Multiphasic Personality Inventory Depression Scale $(r=0.65)$ and Hamilton Rating Scale for Depression (range of $r$ is 0.68-0.76; Biggs, Wylie, \& Ziegler, 1978; Knight, WaalManning, \& Spears, 1983).

\section{Suicide Behaviors Questionnaire-Revised}

The Suicide Behaviors Questionnaire-Revised (SBQ-R; Osman et al., 2001; Appendix G) is a brief, 4-item questionnaire that measures different dimensions of past and future suicidality. The questionnaire has been tested among ethnically diverse youth and adults in both clinical and nonclinical settings. Within youth clinical settings, the intercorrelations items ranged from .62 (items $3 \& 4$ ) to .70 (items $1 \& 2$ ), with 
Cronbach's alpha of .88. Within nonclinical settings, the intercorrelations among the items ranged from .48 (items $3 \& 4$ ) to .82 (items $1 \&$ 2), with Cronbach's alpha of .87 . Although this instrument has not been utilized among the Mong/Hmong ethnic population, it was this researcher's clinical and research opinion that the instrument was suitable for this research study as it is not intrusive in capturing participants' past and likelihood of suicidality.

\section{Mong/Hmong Kev Ntseeg Instrument}

The Mong/Hmong Kev Ntseeg Instrument (MKNI; Appendix H) is a 12-item instrument developed by this researcher to measure the traditional and spiritual healing beliefs of Mong/Hmong participants. The first two questions asked the participants to identify their personal and family religious orientation and the third question asked the participants to identify the number of traditional Mong/Hmong ceremonies they attended during the past year. The last nine questions are rated on a 5-point Likert scale from 1 (Strongly disagree) to 5 (Strongly agree). Due to nonexistence of a Shamanism/Animism measurement and the uniqueness of how psycho-sociocultural issues as well as death are interpreted in traditional Mong/Hmong culture as it relates to spirituality, it was imperative that the spirituality aspect of traditional Mong/Hmong religious practice be examined in this study.

The mean of the last nine items provides a global score; the higher the score the greater the belief in Shamanism/Animism while lower scores reflect no belief in Shamanism/Animism. The highest possible score is 45 . This new instrument was only tested among this sampled population; therefore, this researcher is unable to report the 
validity and reliability of the measurement beyond this study. The reliability of this instrument was conducted using Cronbach's alpha coefficient, which reported a score of .85

\section{Resiliency Questions}

Lastly, two open-ended protective factor questions (Appendix I) concluded the data collection process. The questions were designed to explore protective factors of suicide risk. Due to the sensitivity of the research topic, it was vital that the interviewer conclude the data collection process by encouraging the participants to reflect on their resilience to suicide.

\section{Psychometrics of Scales}

The reliability of all five scales was conducted using Cronbach's alpha coefficient; scores above .70 are ideal, though .50 is acceptable for short scales (e.g., less than 10 items; Pallent, 2010). Given the targeted population for this research study, it was vital that reliability tests be conducted to determine whether the scores of the measurements were consistent across raters. In this study, the FCS (Lee et al., 2000) reported a very good reliability score of .92 with 20 items. The MEIM (Phinney, 1992) reported a good reliability score of .86 with 12 items. The SL-ASIA (Suinn et al., 1992) reported a reliability score of .67 with 21 items. The SDS (Zung, 1965) reported a reliability score of .73 with 19 items. The SBQ-R (Osman et al., 2001) reported a reliability score of .85 with nine items. Lastly, the MKNI reported a reliability score of .85 with nine items. 


\section{Pilot Testing}

Patten (2004) stated that pilot studies are essential in helping researchers determine how new procedures or data collection processes affect vulnerable populations. Therefore, this researcher conducted a pilot test to ensure: (a) participants' comfort in responding to the instruments, (b) comprehension of research materials, and (c) that data collection methods were successful (see Appendix K for pilot testing process).

Eight Mong/Hmong youth (i.e., three males and five females) between the ages of 18 and 25 were invited to participate in this pilot test. Prior to completing the pilot study, the participants were informed of the research study and reviewed and provided feedback on the informed consent. This researcher met individually with each participant to complete the questionnaires. Participants were told that they could make up their answers to the questionnaires and that the primary goal of the pilot study was to gather their feedback on the questions and data collection procedures. After the completion of the materials, this researcher met individually with the participants and discussed the measurement tools as well as the data collection process, thus determining the most effective and culturally appropriate form of data collection tools and methods for the study (e.g., self-administered versus face-to-face interview format with the PI).

During the pilot test, participants took an average of 20 minutes to complete the questionnaires. All participants agreed that the format of the data collection process and the measures were understandable. Additionally, all participants reported that they felt more comfortable responding to the self-administered questionnaires than in a face-toface interview with this researcher. Therefore, during the research study, the participants 
were allowed to choose self-administration or face-to-face interview when completing the questionnaires.

The pilot testing further determined that modifications were needed to one of the instruments in order to capture the participants' appropriate cultural and ethnic use of languages. For example, the ethnic term "Mong/Hmong" was added to one of the instruments and the term "Oriental" was deleted from certain questionnaires due to lack of cultural sensitivity/appropriateness of terminology. Overall, the measures were successfully piloted with eight Mong/Hmong young adults, and the study was revised according to the pilot feedback.

\section{Data Collection Process}

Once the interview dates, time, and location of preference were scheduled, the final data collection process consisted of the following procedure (see Appendix L for recruitment/data collection process):

1. Following informed consent (see Appendix A), this researcher asked whether the participants would like to complete the instruments as self-administered questionnaires or through a face-to-face interview. Furthermore, this researcher encouraged the participants to answer all items honestly and to the best of their knowledge and experience. As this researcher is of the Hmong ethnic background and is fluent in the Hmong language, she was able to administer the questionnaire in both the English and Hmong languages if any of the participants asked for language assistance. 
2. This researcher familiarized the participants with the instruments and informed them that they would receive a $\$ 5$ cash incentive after completion of the instruments. The majority of the participants donated their $\$ 5$ to the campus Mong/Hmong student organization.

3. This researcher asked the participants to pose any questions prior to responding to the instruments.

4. The data collection process took approximately 20 minutes. Once the instruments were completed and collected, the researcher thanked the participants and provided them with a counseling service referral list, the researcher's business card, and flyers to distribute to other Mong/Hmong students who fit the research criteria.

\section{Data Analysis}

Once the socio-demographic survey and measurements were collected by this researcher, the data were entered into the Statistical Package for the Social Sciences (SPSS) for analysis purposes. Correlation and multiple regression statistical analyses were utilized to explore the research questions in this study. The two protective factor questions were analyzed with NVivo10 software. This software supports non-quantitative and mixed method research studies. Thus, coding was applied in the analyses to identify major themes in order to provide preliminary data on suicide protective factors among Mong/Hmong youth (see Appendix $\mathbf{J}$ for outline of Research Questions and Analyses). 


\section{Data Screening}

In analyzing the data, it was essential that the data set be checked for errors and missing data (Agresti \& Finlay, 1997); therefore, the data screening process was utilized to (a) check for error(s), (b) locate the error(s) in the data file, and (c) correct the error(s) in the data file. Once it was determined that there were no errors in the dataset, descriptive statistics (i.e., mean, standard deviation, range of scores) were calculated to describe the characteristics of the sample and check the variables for any violation of assumptions per the statistical techniques chosen to address the research questions (Agresti \& Finlay, 1997). Descriptive statistics were utilized in exploring the first research question.

\section{Correlational Analyses}

The statistical analysis of the Pearson correlation coefficient was utilized to determine the relationships (positive or negative) for the following research questions:

Question 2: Is there an association between ethnic identity and depression among Mong/Hmong youth?

Question 3: Is there a relationship between intergenerational family conflict, traditional and spiritual healing belief system, and ethnic identity among Mong/Hmong youth?

This researcher was mindful that there are multiple issues in utilizing the correlation analysis to determine the associations between variables due to the following: (a) the effect of non-linear relationships, (b) outliers, (c) restriction of range, (d) correlation versus causality, and (e) statistical versus practical significance. For these 
purposes, it was essential that the assumptions for correlations were met: (a) level of measurement (continuous variables), (b) related pairs (each participant must respond to both variables), (c) independence of observation, (d) normality, (e) linearity, and (f) homoscedasticity (Pallent, 2005).

Once the correlations of the variables were computed, the statistic obtained was the Pearson's product-moment correlation $(r)$, which indicates the strength and direction of the linear relationship between the variables. The computed relationship between the variables was examined using a scatterplot as it provides a visual indication of the strength and direction of the relationship. Additionally, the correction analysis further examined for outliers, issues with normality, constant variance, homoscedasticity, and independence of observation to ensure that the assumptions in regression testing were not violated.

\section{Multiple Regression}

The multiple regression analysis was utilized to answer the following research questions:

Question 4: How well do acculturation, ethnic identity, and intergenerational family conflict predict depression among Mong/Hmong youth?

Question 5: What are the effects of ethnic identity, intergenerational family conflict, depression, family household size, and traditional and spiritual healing beliefs on suicide risk among Mong/Hmong youth?

According to Tabachnick and Fidell (2001), the multiple regression model is an appropriate statistical analysis to use when exploring the relationship between one 
continuous dependent variable and a number of continuous independent variables. The model provides information on how much of the variance in the dependent variable is influenced by the independent variables as well as the contribution of each independent variable.

Prior to conducting the multiple regression, correlations were computed to explore the relationships among both the dependent and independent variables. The assumptions of multiple regression were reviewed prior to conducting the analysis as related to sample size, multicollinearity and singularity, outliers, normality, linearity, homoscedastically, and independence of residuals.

In the multiple regression model, all the independent variables were entered into the equation simultaneously to determine: (a) how well the independent variables predicted the dependent variables, and (b) an indication of the unique variance of how each independent variable influenced the dependent variable. Once the multiple regression analysis was computed, this researcher checked the assumptions to ensure that no violations occurred. An inspection of the correlation on the data analysis determined whether multicollinearity (less tolerance than .10) occurred, which would be a violation. Furthermore, the scatterplot and an inspection of the Mahalanobis indicated whether outliers were present in the data. Fortunately, there were no violations of assumptions; thus, there was no need to manipulate the data.

To determine the predictive outcomes for the research questions four and five (multiple regression), the following output from the analysis provided the answer: (a) the overall model significant value $(p<.05)$; (b) the variance $\left(\mathrm{R}^{2}\right)$ in the dependent variable, 
accounted for by the model; (c) F-test; (d) beta coefficients (i.e., variable's contribution to the prediction of the dependent variable); and (e) the significant value among the predictors $(p<.05)$.

NVivo 10

Two open-ended protective factor questions (see Appendix I) concluded the data collection process. The questions were designed to explore protective factors of suicide risk. The questions were: (a) If you have had thoughts of ending your life, how did you respond to them (or deal with them), and (b) From your perspective, what would be most helpful to overcome having thoughts of ending one's life? The majority of responses were stated in short phrases or less than one sentence, so the coding of the responses was easily extracted to form major themes by analyzing the results via NVivo 10.

\section{Missing Data}

Data were inspected using descriptive statistics to determine the percentage of missing values for each scale. Four scales reported less than $5 \%$ missing values, so no action was taken with these items. After careful inspection and consideration, regression imputation technique was conducted on 13 items of the SDS, the percent of missing values ranged from $1 \%$ to $8 \%$. The total score of the SDS was calculated and labeled as the "depression" variable for this study. Depression was utilized as both an independent and dependent variable in this study (research questions two and four). Ideally, for social science research purposes, it is not desirable to retain dependent variables with high missing values (20\% or less is acceptable; Little \& Rubin, 2002). It should be noted that high missing values impact research studies with regard to internal validity, external 
validity, parameter estimate accuracy, standard errors bias, and population means. Therefore, regression imputation methods were used to replace missing values, thereby increasing the number of cases analyzed and allowing for inference regarding a population of interest.

\section{Regression Imputation}

Regression imputation was selected as the best method to replace missing data for several reasons: (a) missing values along the scale were distributed across several items; (b) the method can generate unbiased estimates of means; (c) the associations add regression coefficients in a much wider range of settings than a simple mean imputation; and (d) the method uses information from missing cases to predict missing values, providing acceptable estimates of missing values (Kenward \& Carpenter, 2007).

To conduct regression imputation, a Pearson product-moment correlation coefficient was generated that identified three of the highest correlated predictor variables (independent variables) associated with the imputed item (dependent variable). The highly correlated predictors were selected and used in the regression equation $(y=a+$ $b x 1+b x 2+b x 3)$

After identifying the independent and dependent variables, a regression analysis was conducted. From the regression analyses, the researcher calculated the regression equation using unstandardized values for $y$-intercept and slopes, $y=a+b x 1+b x 2+b x 3$. Only cases with complete data for the predictor variables were utilized to generate the regression equation. Values from missing cases were inserted into the dataset, thus decreasing the missing data percentage. The regression imputation method was repeated 
until there was minimal "difference between the predicted values from one step to the next" (Saunders et al., 2006, p. 23).

Of the 20 items on the SDS, two items were removed. The items removed were Sex ("I still enjoy sex") and Constip ("I have trouble with constipation"). Of the 165 participants, only $133(80 \%)$ responded to the item Sex and $150(90 \%)$ responded to the item Constip. Due to the targeted population sample (cultural) and age group, it was appropriate that both Sex and Constip were excluded from the scale. It was reasoned that the subject matters of sexual behavior and physical manifestation (e.g., constipation) were considered culturally taboo topics of discussion and reporting, which might explain the missing values for these two items.

Once the two items (i.e., Sex and Constip) were removed, the number of valid cases increased to 141 , a total of $15 \%$ missing values across the SDS. Although the missing value decreased due to the removal of the two items above, a total of $15 \%$ missing value was not acceptable to this researcher; thus, the regression imputation method as outlined was applied to this dataset. After conducting regression imputation, the final output indicated a valid total of 5\% missing value, which was acceptable. 


\section{Chapter 4: Results}

This chapter contains the findings used to determine whether there were any correlations or significant predictors of depression or suicide risk among Mong/Hmong youth between the ages of 18 and 25. Data were entered and analyzed using SPSS. First, analyses were performed to determine data errors, missing data, descriptive statistics (i.e., rate of suicide ideations and characteristics of the sampled population), and violations of assumptions based on the selected statistical analyses of research questions. Second, the psychometric properties of each scale were assessed to determine the reliability of given sampled populations. Third, statistical analysis of the Pearson product-moment correlation coefficient was utilized to determine the relationship between ethnic identity and depression, as well as whether any association existed between intergenerational family conflict, spiritual belief system, and ethnic identity among the sampled population. Fourth, multiple regression statistical analyses were performed to determine the predictors of depression and suicide risk. Finally, the coding of the protective factor responses was easily extracted by analyzing the results via NVivo 10 to form major themes and provide preliminary data among Mong/Hmong youth.

\section{Preliminary Analysis}

Preliminary analysis was conducted to identify data entry errors and missing cases. Once the errors were corrected, descriptive statistics (i.e., mean, standard deviation, range of scores) were determined to (a) describe characteristics of the sample population, and (b) check variables for any violation of assumptions based on statistical techniques addressing research questions. Descriptive statistics were observed for 
continuous variables; output provided information regarding the distribution of scores (i.e., skewness, kurtosis), standard deviation, and mean. Normality assessment was conducted to measure distribution of scores on the dependent variables; all outputs indicated normality (i.e., symmetrical, bell-shaped curve, skewness, kurtosis).

\section{Participant Demographics}

A total of $165 \mathrm{Mong} / \mathrm{Hmong}$ youth between the ages of 18 and 25 were recruited to participate in this study. Of the 165 participants, 82 were male and 83 were female; 120 of the participants were born in the United States, 40 were born in Thailand, and five were born in other counties. The college levels of the participants $(N=165)$ were: 45 freshmen, 48 sophomores, 27 juniors, and 45 seniors. The majority of the participants reported marital status as single $(n=162)$; job status as unemployed $(n=89)$; annual income between $\$ 1,000$ to $\$ 5,000$; and being raised by both biological parents $(n=$ 154).Regarding current living arrangements, participants reported the following: 123 resided with family members, 37 resided with a friend/roommates, and five resided alone or with a significant partner. Table 1 provides additional data on the sample population. 
Table 1

Demographic Characteristics of Participants

\begin{tabular}{|c|c|c|}
\hline Variable & $N$ & $\%$ \\
\hline \multicolumn{3}{|l|}{ Gender } \\
\hline Female & 82 & 49.7 \\
\hline Male & 83 & 50.3 \\
\hline \multicolumn{3}{|l|}{ Age } \\
\hline 18 & 32 & 19.4 \\
\hline 19 & 42 & 25.5 \\
\hline 20 & 21 & 12.7 \\
\hline 21 & 29 & 17.6 \\
\hline 22 & 25 & 15.2 \\
\hline 23 & 5 & 03.0 \\
\hline 24 & 3 & 1.8 \\
\hline 25 & 6 & 3.6 \\
\hline Missing & 2 & 1.2 \\
\hline \multicolumn{3}{|l|}{ Birth Country } \\
\hline U.S.A. & 120 & 72.7 \\
\hline Thailand & 40 & 24.2 \\
\hline Other & 5 & 3.0 \\
\hline \multicolumn{3}{|l|}{ College Level } \\
\hline Freshman & 45 & 27.3 \\
\hline Sophomore & 48 & 29.1 \\
\hline Junior & 27 & 16.4 \\
\hline Senior & 45 & 27.3 \\
\hline \multicolumn{3}{|l|}{ Sexual Orientation } \\
\hline Heterosexual & 162 & 98.2 \\
\hline Homosexual & 1 & 00.6 \\
\hline Bisexual & 1 & 00.6 \\
\hline Not Sure & 1 & 00.6 \\
\hline \multicolumn{3}{|l|}{ Marital Status } \\
\hline Single & 162 & 98.2 \\
\hline Married & 3 & 1.8 \\
\hline
\end{tabular}


Table 1 (continued)

Demographic Characteristics of Participants

\begin{tabular}{|c|c|c|}
\hline Variable & $N$ & $\%$ \\
\hline \multicolumn{3}{|l|}{ Employment Status } \\
\hline Employed & 76 & 46.0 \\
\hline Unemployed & 89 & 53.9 \\
\hline \multicolumn{3}{|l|}{ Living Arrangement } \\
\hline Self & 4 & 2.4 \\
\hline Family & 123 & 74.5 \\
\hline Friend(s)/Roommate(s) & 37 & 22.4 \\
\hline Significant Partner & 1 & 0.6 \\
\hline \multicolumn{3}{|l|}{ Family Household Size } \\
\hline 1 to 3 & 4 & 2.4 \\
\hline 4 to 6 & 29 & 17.6 \\
\hline 7 to 9 & 61 & 37.0 \\
\hline 10 to 12 & 52 & 31.5 \\
\hline $12+$ & 19 & 11.5 \\
\hline \multicolumn{3}{|l|}{ Religion } \\
\hline Shamanism/Animism & 121 & 73.3 \\
\hline Christian Faith & 21 & 12.7 \\
\hline Mormon & 2 & 1.2 \\
\hline Other Religion & 11 & 6.7 \\
\hline More than One Religion & 10 & 6.1 \\
\hline \multicolumn{3}{|l|}{ Marital Status of Parents } \\
\hline Married & 134 & 81.2 \\
\hline Divorced & 9 & 5.5 \\
\hline Separated & 4 & 2.4 \\
\hline Widowed & 15 & 9.1 \\
\hline Deceased & 2 & 1.2 \\
\hline Missing & 1 & 0.6 \\
\hline \multicolumn{3}{|l|}{ Parent’s U.S. Citizenship Status } \\
\hline Both Parents U.S. Citizens & 32 & 19.4 \\
\hline Only 1 Parent is U.S. Citizen & 42 & 25.5 \\
\hline
\end{tabular}


Table 1 (continued)

Demographic Characteristics of Participants

\begin{tabular}{lcc}
\hline Variable & $N$ & $\%$ \\
\hline Not Naturalized & 17 & 10.3 \\
Missing & 74 & 44.8 \\
Parent Employment Status & & \\
Employed & 92 & 55.7 \\
Unemployed & 72 & 43.6 \\
Missing & 1 & 0.6 \\
Family Income & & \\
\$10,000 to $\$ 20,000$ & 87 & 52.7 \\
$\$ 20,000$ to $\$ 30,000$ & 41 & 24.8 \\
$\$ 30,000$ to $\$ 40,000$ & 9 & 5.5 \\
$\$ 40,000+$ & 21 & 12.7 \\
Missing & 7 & 4.2 \\
\hline Note. Total $N=165$ & &
\end{tabular}

Note. Total $N=165$.

\section{Research Question 1}

The first research question determined the rate of suicide ideation and attempts among the sampled population (see Table 2). The hypothesis was that at least $50 \%$ of the participants would report they had thoughts of suicide ideation and attempts. In order to determine the rate of suicide ideation and attempts, the overall result from the SBQ-R, item 1 was examined.

Table 2

Lifetime Suicide Ideation and/or Suicide Attempts

\begin{tabular}{lcc}
\hline Answer & $N$ & $\%$ \\
\hline Never (Non-Suicidal Subgroup) & 65 & 39.4 \\
Brief Passing Thought (Suicide Risk Ideation & 98 & 59.4 \\
Subgroup) & & \\
Have a Plan (Suicide Plan Subgroup) & 0 & 00.0 \\
Have Attempted (Suicide Attempt Subgroup) & 2 & 1.2 \\
Total & 165 & 100 \\
\hline
\end{tabular}


Of the 165 respondents, $59 \%(n=98)$ reported that they have had a brief passing thought of killing themselves and $.01 \%(n=2)$ indicated that they attempted suicide. Overall, 60\% $(n=100)$ of the respondents had a lifetime experience of suicide ideation and/or suicide attempts.

\section{Research Question 2}

The second research question examined whether there was an association between ethnic identity and depression among Mong/Hmong youth. Previous studies on ethnic identity and psychosocial behavioral outcomes reported that individuals with insufficient ethnic identification are more likely to develop behavioral issues such as depression; it was therefore hypothesized that depression among this sampled population would increase with decreasing ethnic identity. Table 3 reports the correlation between ethnic identity and depression found in this study.

Table 3

Pearson Product-Moment Correlations Between Ethnic Identity and Depression

\section{Ethnic Identity}

Depression $\quad-.41^{* *}$

$* * p<.01$ (2-tailed).

The relationship between ethnic identity and depression was investigated using Pearson product-moment correlation coefficient. Preliminary analyses were performed to ensure there was no violation of the assumptions of normality, linearity, and homoscedasticity. Results indicated a moderate, negative correlation between the two variables, $r=-.41, n=158, p<.01$, with low levels of depression associated with high 
levels of ethnic identity achievement. The data suggest that participants with higher levels of ethnic identity reported lower levels of depression.

\section{Research Question 3}

The third research question examined the relationships between intergenerational family conflict, Mong/Hmong traditional and spiritual healing beliefs, and ethnic identity. This researcher hypothesized that intergenerational family conflict would increase with decreasing ethnic identity and Mong/Hmong traditional and spiritual healing beliefs. According to current literature on intergenerational family conflict, family conflict increases as individuals distance themselves from ethnic identity and family belief systems. Table 4 reports the correlations between intergenerational family conflict, Mong/Hmong traditional and spiritual healing beliefs, and ethnic identity.

Table 4

Pearson Product-Moment Correlations Between Intergenerational Family Conflict, Traditional \& Spiritual Healing Beliefs, and Ethnic Identity

\begin{tabular}{ccc}
\hline Family Conflict & Mong/Hmong & Ethnic Identity \\
& Traditional \& & \\
& Spiritual Healing & \\
& Beliefs & \\
\hline
\end{tabular}

Family Conflict

Mong/Hmong .09

Traditional \&

Spiritual Healing

Beliefs

Ethnic Identity

$-.04$

$.39 * *$

$* * p<.01$ (2-tailed).

The Pearson product-moment correlation coefficient was utilized to examine the relationships between all three variables (i.e., intergenerational family conflict, Mong/Hmong traditional and spiritual healing beliefs, and ethnic identity). Results 
indicated a moderate, positive correlation between traditional and spiritual healing belief and ethnic identity, $r=.39, n=165, p<.01$. The data suggest that when individuals achieve ethnic identity, they have a greater belief in the Mong/Hmong traditional and spiritual healing practices.

\section{Research Question 4}

The fourth research question examined how well acculturation status, ethnic identity, and intergenerational family conflict predicted depression. The researcher hypothesized that high intergenerational family conflict, low ethnic identity, and low acculturation would predict depression among Mong/Hmong youth. Individuals who distance themselves from their family's ethnic identity are more likely to experience increased intergenerational family conflict and are more likely to separate from their cultural identity, thus leading to a depressive behavioral outcome.

Prior to examining whether the variables of acculturation status, ethnic identity, and intergenerational family conflict predicted depression among Mong/Hmong youth, a Pearson product-moment correlation coefficient was conducted to examine the relationships among these variables. Results indicated that ethnic identity and intergenerational family conflict are associated with depression (see Table 5). 
Table 5

Pearson Product-Moment Correlations Between Depression, Acculturation, Ethnic Identity, and Intergenerational Family Conflict

\begin{tabular}{lcccc}
\hline & Depression & Acculturation & Ethnic Identity & $\begin{array}{c}\text { Intergenerational } \\
\text { Family Conflict }\end{array}$ \\
\hline Depression & .073 & & \\
Acculturation & $-.393^{* *}$ & $-.357^{* *}$ & & \\
Ethnic Identity & $.298^{* *}$ & .114 & -.030 & \\
Intergenerational & & & & \\
Family Conflict & & & & \\
\hline$* * p<.01(2-$ tailed $)$ & & &
\end{tabular}

** $p<.01$ (2-tailed).

After reviewing the correlation, multiple regression analysis was performed to determine predictors of depression. Table 6 reports the multiple regression analysis that determined whether acculturation, ethnic identity, and intergenerational family conflict were significant predictors of depression among Mong/Hmong youth.

Table 6

Multiple Regression Analyses for Variable Predicting Depression among Mong/Hmong Youth $(N=154)$

\begin{tabular}{lccc}
\hline Predictor & $\mathrm{B}$ & $\mathrm{SE}$ & $\beta$ \\
\hline Acculturation & -2.88 & 1.96 & -.112 \\
Ethnic Identity & -6.94 & 1.24 & $-.424^{* *}$ \\
Intergenerational & 0.14 & 0.03 & $.298^{* *}$ \\
Family Conflict & & & \\
$\mathrm{R}^{2}$ & & 0.25 & \\
$\mathrm{~F}$ & & $16.47^{* *}$ & \\
\hline$* * p<.01$ (2-tailed). & & &
\end{tabular}

Results of the regression analysis indicated that ethnic identity $(b=-6.94, \mathrm{SE} b=$ $1.24, \beta=-.424, p<.01)$ and intergenerational family conflict $(\mathrm{b}=0.14, \mathrm{SE} b=0.03, \beta=$ $.298, p<.01)$ were related significantly to depression. After accounting for the predictive effects of acculturation and ethnic identity on depression, youth who experienced high 
intergenerational family conflict were more likely to have higher depression rate. After accounting for acculturation and intergenerational family conflict, youth who achieved a high ethnic identity were expected to have a low depression rate. Overall, both ethnic identity and intergenerational family conflict accounted for $25 \%$ of the variance in depression, $\mathrm{R}^{2}=0.25, F(3,150)=16.47, p<.01$.

\section{Research Question 5}

The fifth research question determined the effects of ethnic identity, intergenerational family conflict, depression, family household size, and Mong/Hmong traditional and spiritual healing beliefs on suicide risk among Mong/Hmong youth. The hypothesis was that low ethnic identity, high intergenerational family conflict, and low depression would predict suicide risk among Mong/Hmong youth. Individuals with low self-identification of ethnicity are more likely to report high intergenerational family conflict, thus leading to increased depression and, eventually, suicidal behavior.

A Pearson product-moment correlation coefficient and multiple regression analyses were conducted. Results from the Pearson product-moment correlation coefficient indicated a relationship between ethnic identity, intergenerational family conflict, depression, and Mong/Hmong traditional and spiritual healing beliefs (see Table 7). 
Table 7

Pearson Product-Moment Correlations Between Suicide Risk, Ethnic Identity, Intergenerational Family Conflict, Depression, Family Household Size, and Mong/Hmong Traditional \& Spiritual Healing Beliefs

\begin{tabular}{|c|c|c|c|c|c|c|}
\hline & $\begin{array}{l}\text { Suicide } \\
\text { Risk }\end{array}$ & $\begin{array}{l}\text { Ethnic } \\
\text { Identity }\end{array}$ & $\begin{array}{c}\text { Intergenerational } \\
\text { Family Conflict }\end{array}$ & Depression & $\begin{array}{c}\text { Family } \\
\text { Household } \\
\text { Size }\end{array}$ & $\begin{array}{l}\text { Spiritual } \\
\text { Beliefs }\end{array}$ \\
\hline \multicolumn{7}{|l|}{ Suicide Risk } \\
\hline Ethnic Identity & $-.189 * *$ & & & & & \\
\hline $\begin{array}{l}\text { Intergenerational } \\
\text { Family Conflict }\end{array}$ & $.175^{*}$ & -.057 & & & & \\
\hline Depression & $.436 * *$ & $-.405^{* *}$ & $.317 * *$ & & & \\
\hline $\begin{array}{l}\text { Family Household } \\
\text { Size }\end{array}$ & .054 & -.080 & $-.047 *$ & -.111 & & \\
\hline Spiritual Beliefs & $.164 *$ & $.389 * *$ & .079 & -.020 & -.033 & \\
\hline
\end{tabular}

$* p<.05 ; * * p<.01$ (2-tailed).

Table 8 reports the results of the regression model, which indicates that depression and traditional and spiritual healing beliefs were significantly related to suicide risk: depression, $\mathrm{b}=.091, \mathrm{SE} \mathrm{b}=.019, t=4.808, \beta=.399, p<.01$; and traditional and spiritual healing beliefs, $\mathrm{b}=.515, \mathrm{SE} \mathrm{b}=.190, t=.211, \beta=.211, p<.01$.

Table 8

Multiple Regression Analyses for Variables Predicting Suicide Risk among Mong/Hmong Youth $(N=158)$

\begin{tabular}{lccc}
\hline Predictor & $\mathrm{B}$ & $\mathrm{SE} \mathrm{b}$ & $\beta$ \\
\hline Ethnic Identity & -.373 & .324 & -.099 \\
Intergenerational & .004 & .008 & .041 \\
Family Conflict & & & \\
Depression & .091 & .019 & $.399^{* *}$ \\
Family Household & .185 & .130 & .103 \\
Size & & & $.211^{* *}$ \\
Spiritual Beliefs & .515 & .190 & \\
$\mathrm{R}^{2}$ & & .240 & \\
$\mathrm{~F}$ & & $9.613^{* *}$ & \\
\hline$* * p<.01$ (2-tailed). & & &
\end{tabular}


After controlling for the other variables in the model, every reported increase in depression was correlated to a $40 \%$ increase in suicide risk. Additionally, having controlled for ethnic identity, family intergenerational conflict, depression, and family size, the results indicated that suicide risk increased by $21 \%$ among individuals with a strong belief in the Mong/Hmong traditional and spiritual healing practices. Ethnic identity, intergenerational family conflict, and family household size did not independently predict suicide. Overall, both depression and Mong/Hmong traditional spiritual and healing beliefs accounted for $24 \%$ of the variance in suicide risk, $\mathrm{R}^{2}=0.24$, $F(3,152)=9.613, p<.01$.

\section{Protective Factors}

Two open-ended protective factor questions concluded the data collection process. The questions were designed to explore protective factors of suicide risk. The questions were:

1. If you have had thoughts of ending your life, how did you respond to them (or deal with them)?

2. From your perspective, what would be most helpful to overcome having thoughts of ending one's life?

The coding of the responses was easily extracted to form major themes by analyzing the results via NVivo 10.

\section{Coping With Thoughts of Ending Life}

A total of four major themes emerged related to how participants responded to dealing with thoughts of ending their life. These themes were: (a) realizing how death 
impacts loved ones, (b) optimism, (c) connectedness (i.e., family, friends, and community support), and (d) having self-worth.

Impact among loved ones. The majority of participants identified the thought of how their death would impact loved ones to be a common theme in how they responded to not ending their life. In utilizing NVivo 10, this researcher identified a total of 35 references pertaining to the theme of how their death would impact "loved ones." Participants shared similar thoughts such as, "I thought about my mom and how she will live if I die" to "I've thought of killing myself, but I always think of the people that loves me. I always think of how they would feel or just the thought of seeing them cry would stop me from thinking about death."

Optimism. Optimism and having a positive orientation toward the future was another common theme. A total of 21 references were coded under this theme. Participants expressed that they thought about their future, remained positive, and relied upon their goals and dreams in order to move forward and deter the thoughts of ending their life. For instance, one participant stated, "Think positive life is too short. Others wish to live, why do we choose to die?" Another participant shared, "Think about the future and opportunity that I still have.” Participants were very expressive under this theme as many used terminology such as happy, future, positive, opportunity, and dreams to describe how they dealt with previous thoughts of ending their life.

Connectedness. Connectedness through communication and social interaction with family, friends, and community was the third leading theme identified in how the participants responded in dealing with thoughts of ending their life. A total of 18 
references were coded under this theme. Participants report that talking to people such as family, friends, teachers, therapists, and anyone who was willing to listen; in addition to staying active, helped them process their thoughts and deterred them from ending their life. For example, one participant stated, "I talk to someone (anyone) to put things out of my system." Another participant reported, "I just never talked about it until I feel I can trust someone." Overall, the participants who identified that connectedness was a key factor to their response in not completing suicidality were very vocal in that talking and engaging in social activities helped them to refrain from thoughts of ending their life.

Self-worth. A total of 17 references were identified and coded under self-worth. Participants expressed life as being valuable and worthy; thus, these thoughts deterred them from ending their life. For instance, the participants made several common statements regarding self-worth: ". . . there is always a reason to live;" "... I know that life is precious;" "I tell myself that life may be hard for me, but I may still be worthy;" and "I believe that life is valuable and special even through all the hardships."

\section{Factors to Overcome Suicidality}

A total of three major themes emerged in exploring participants' perspectives in identifying "what would be most helpful to overcome having thoughts of ending one's life." These themes were similar to the participants' responses in how they responded to thoughts of ending their life: (a) thoughts of how one's death would impact loved ones, (b) optimism, and (c) connectedness (i.e., family, friends, community support).

Impact among loved ones. There were 42 references coded in which the participants stated that one should "think/understand/image/realize" how their death 
would impact families and friends. Some examples are the following: "They should think of the people that love them and how those people would live after they pass," "Think about all the tears and pain their love ones will face if they were to end their life," and "Know that the ones you love would not want that before you have thoughts of leaving, always think to yourself about the others. You do NOT want to leave without proper communication and resolvement."

Optimism. Another common factor identified in what would be most helpful to overcome having thoughts of ending one's life was to be optimistic and have a positive orientation toward the future. There were 41 references identified and coded to form this theme. Several participants shared statements indicating that the individual should "think positively" and have a "positive outlook for the future."

Connectedness. The majority of participants stated that communication and social interaction were crucial to overcoming thoughts of ending their life. In utilizing NVivo 10, this researcher identified a total of 87 references coded pertaining to communication (e.g., "talking," "sharing"), family/friends, and social bond. For instance, one participant stated, “The most helpful to overcome having thoughts of ending one's life is to talk to others or loved ones." Another stated, "Be more supportive, understanding and basically communicate more. Families tend to stay stronger through the nurturing of communication." Additionally, a handful of participants referenced seeking a therapist/counselor to "talk about their issues" in order to overcome having thoughts of ending their life. For example, one participant shared the following, "Seeking help -- talking to professional and having someone to listen to your problems." 
Close family and social bonds such as family, friends, and boyfriend/girlfriend were identified as supportive factors in overcoming having thoughts of ending their life. A total of 43 references were identified and coded under this theme. One participant stated, "Having friends and family members who care about you and verbalize it, who accepts you for who you are, and willing to put everything aside just to make you sure you're okay." The majority of responses under this theme referenced "family and friends" together as mentioned in the above statement in addition to the following, "Positive support from their family, peers, friends, community, etc." Although family and friends were frequently mentioned together in the participants' responses, there were a sizable number of references to being in a relationship (with boyfriend/girlfriend) as a supportive factor in overcoming having thoughts of ending their life.

Participation in academic or community programs or activities was another leading protector in overcoming having thoughts of ending their life. According to the results of this study, there were 23 references coded indicating involvement with peers, identifying a hobby, joining a group or organization, and keeping occupied as determents of having thoughts of ending their life. For instance, one participant stated, "Keep your social life open and accepting so that loneliness is not a factor of making one person feel there is no one to care or accept them." Another participant shared, "Get involved with something that is bigger than yourself. Ex: club, sports, orgs." 


\section{Chapter 5: Discussion}

\section{Major Findings}

The primary objective of this study was to examine whether psychological, social, and cultural factors impact suicide risk among Mong/Hmong youth between the ages of 18 and 25 . The following independent variables were utilized to determine their influence on depression and suicide risk (dependent variables) among the sampled population: intergenerational family conflict, ethnic identity, acculturation, depression, and traditional and spiritual healing beliefs. The psychological aspect of the study was measured by depression, the social relation aspect was measured by intergenerational family conflict, and the cultural aspect was measured by acculturation, ethnic identity, and traditional and spiritual healing beliefs.

\section{Rate of Suicide Risk}

Of the 165 respondents, 59\% $(n=98)$ had passing thoughts of suicide. According to Kessler, Borges, and Walters (1999), a national survey on suicidality found the general population rate of lifetime suicidal ideation to be $13.5 \%$ and suicide attempts to be $4.6 \%$.

Of the individuals who have had a suicidal ideation, $34 \%$ reported that they seriously thought about suicide to the point of developing a suicide plan (Kessler et al., 1999). Additionally, $72 \%$ of those who develop a suicide plan report attempting suicide (Kessler et al., 1999). These data suggest that when compared to the general population, this study's population was four times more likely to have experienced lifetime suicidal ideation. 


\section{Correlation Between Depression and Ethnic Identity}

This study was consistent with prior studies that indicated a correlation between ethnic identity and depression (Cheng et al., 2010; Eldering, 1995; Phinney, 1990). Overall, this finding suggests that depression decreases with higher levels of ethnic identity. Although there is limited research on how ethnic identity influences depression and suicide among Asian Americans, the results of this study were consistent with existing research studies conducted on ethnic identity. For instance, Erikson (1950) stated that individuals or groups who successfully develop a sense of self and personal identity have greater opportunities to maintain positive psychological and healthy relationships; however, those who fail are likely to experience psychological and behavioral problems. Additionally, studies conducted by Cheng et al. (2010), Eldering (1995), and Phinney (1990) stated that when an individual connects to his or her ethnic community or reports a higher level of ethnic identity, he or she has lower rates of psychological issues (e.g., depression, suicide attempts).

\section{Correlation Between Healing Belief and Ethnic Identity}

In examining the correlation between intergenerational family conflict, Mong/Hmong traditional and spiritual healing practices, and ethnic identity, results indicated that among the sampled population, individuals who reported a higher level of ethnic identity had a greater belief in the traditional and spiritual healing practices. This suggests that participants who were able to formulate and make sense of their membership within an ethnic group in order to secure their identity within the larger society had a greater belief in the Mong/Hmong traditional and spiritual healing 
practices. For instance, participants who reported a high level of ethnic identity agreed that negative things, such as suicidality, are caused by family curses, spirits, demons and/or ancestors. Additionally, they also believe that the best form of treatment to cure negative thinking is a Mong/Hmong Shaman/Shawoman.

\section{Predictors of Depression}

Ethnic identity and intergenerational family conflict are significant predictors of depression, a psychological mental health disorder that is highly associated with suicidality (Eisenberg et al., 2007; Gencoz \& Or, 2006; Gutierrez et al., 2000). This finding suggests that as intergenerational family conflict increased among the sampled population, depression rates increased; thus, suggesting intergenerational family conflict is a risk factor in predicting depression among Mong/Hmong youth. It is evident that when conflict arises within a family, regardless of ethnicity or years of acculturation, the parties involved are likely to experience some sort of negative behavioral outcomes if there is no resolution to the existing problem. This finding was consistent with prior research indicating that when intergenerational family conflict exists, depression and delinquent behaviors increase (Aldwin \& Greenberger, 1987; Hall, 1987; Su et al., 2005). Additionally, among Asian American families, intergenerational family conflict is a major contributor to para-suicidal behavior and the completion of suicide, even when controlling for depression (Cheng et al., 2010; Lo, 2009; Lorenzo et al., 1995).

\section{Predictors of Suicide Risk}

Results indicated that depression and traditional and spiritual healing beliefs were predictors of suicide risk among the sampled population even though there was little risk. 
This finding of depression as a predictor of suicide risk was consistent with existing literature that indicated depression to be a primary risk factor in suicidality (Eisenberg et al., 2007; Gencoz \& Or, 2006; Gutierrez et al., 2000). Interestingly, the findings from this sampled population reported that suicide risk increased by $21 \%$ among individuals with a strong belief in the traditional and spiritual healing practices.

Readers must take into consideration that of the 165 respondents, 121 selfreported their spiritual orientation as Shamanism/Animism, the traditional religious belief of the Mong/Hmong population. As the majority of respondents identified themselves as Shamanism/Animism (73.3\%), there was bias in the reporting; the stronger belief in traditional Mong/Hmong spiritual/healing practices, the more likely an individual is of the Shamanism/Animism religion. Due to the uniqueness of the traditional Mong/Hmong religious belief system and the spiritual interpretation of death, when spiritual (i.e., ancestors or evil spirits) needs are unmet, death awaits the individual and once deceased he or she will eventually be reincarnated (Fadiman, 1997; Siegal \& McSilver, 2001). There is a religious belief that those who are deceased and have unmet spiritual needs will continue to hunt and bring illnesses to their loved ones until their spiritual needs are met. Once the spiritual needs are met, the deceased will be reincarnated to another living being, creature, or natural objects (e.g., rocks, water, sand, etc.). Therefore, the findings may suggest that individuals who hold a strong belief in the traditional Mong/Hmong spirituality do not view suicidality as a sin, but rather as a virtue; therefore, these individuals may view suicide as an act of intentionally ending their own life in order to seek the beginning of a new and better life through reincarnation. 


\section{Protective Factors}

Several protective factors emerged in how the participants responded to having thoughts of ending their life and how they overcame those thoughts. These protective factors were similar to published literature indicating that having family cohesion, community support, trait optimism, and support from medical and health care personnel are keys to reducing the potential for suicidal behavior (Center for Substance Abuse Treatment, 2009; U.S. Public Health Service, 1999). The result of recognizing guilt and realizing the impact of how one's death would affect loved ones as a protective factor was surprisingly notable as research studies have shown that Asian youth are more likely to commit suicide as they are unable to live up to parental expectations (Jesilow \& Xiong, 2007; Lee et al., 2005; Sue, 2004; Uba, 1994). As mentioned in the literature review, many traditional Asian cultures, such as the Mong/Hmong, embody a tacit understanding that failure is not within the individual, but within the family unit. The result from this sampled population indicated that shaming the family or "losing face" may be a protective factor of suicide risk (Jesilow \& Xiong, 2007; Sue, 2004; Uba, 1994).

\section{Limitations}

Several limitations existed within this research study. First, the recruitment sites were limited to university settings, so the results cannot be generalized to all Mong/Hmong youth in the state of California or within the United States. Second, the terminology of "youth" applied in this study to individuals between the ages of 18 and 25. Readers are cautioned not to generalize the findings to individuals outside of this ethnicity and age group. Third, none of the standardized instruments were specifically 
developed to measure the uniqueness of the Mong/Hmong population; therefore, the data may not be reflective of the participants' cultural perceptions of themselves or their family values. Fourth, the SL-ASIA (Suinn et al., 1992) was slightly altered to include the targeted ethnic group, Mong/Hmong. The term "Mong/Hmong" was inserted into the survey in order to provide the participants a sense of ethnic identity. As mentioned in the literature review, the Mong/Hmong differ tremendously from other Asian subgroups; thus, the interchange and inclusion of the term "Mong/Hmong" in this instrument may not have yielded equivalent results. Lastly, this study examined predictive factors of Mong/Hmong youth suicide risk; therefore, there remains a variety of unknown predictors beyond those presented in this study that could contribute to suicide risk among this population. The generalizability of the findings beyond the sample and independent variables should be conducted with caution.

\section{Implications to Social Work}

The outcomes of this study have several implications for the field of social work practice and policy. As a result, this section describes implications and recommendations at the micro (i.e., individual/local), meso (i.e., group), and macro levels (i.e., social/global) in order to decrease suicidal risk and address and improve the mental health system for Mong/Hmong youth.

\section{Practice}

The social problem of Mong/Hmong youth suicide attempts is an emerging issue and changes in how the mental health care system responds to this population are needed. At the local level, community resources and funding to support Mong/Hmong training 
programs in the area of mental health and education must be addressed in order for community providers to better understand the cultural norms of this population.

Furthermore, community-based treatment options for ethnic minorities, such as the Mong/Hmong population, must expand; this expansion will create alternative treatment programs to include traditional Mong/Hmong mental health treatment and reduce unnecessary institutionalization. Lastly, community-based mental health care services should develop a working relationship and collaborate with established Mong/Hmong community organizations to build a culturally sensitive mental health care system to adequately serve this population in order to address mental health issues and treatment within this closely-knit community.

At the state level, there is a need to diversify the mental health delivery system to include ongoing research and clinical practice to better understand the mental health needs of the Mong/Hmong population and how agencies are delivering mental health services to disadvantaged populations. There is a definite need to further address the racial and ethnic disparities in mental health access. Moreover, state leaders must set standards and continuously evaluate cultural competence in the mental health system. As such, research into the effectiveness of service delivery and needs of Mong/Hmong children and youth in the mental health care system must continuously be conducted in order to ensure that community needs are being met and services are producing positive outcomes.

Finally, at the federal level, national education regarding the mental health of children and youth among all racial and ethnic groups must be initiated. Educating racial 
and ethnic communities on mental illness should be a priority in order to educate ethnic groups about the facts and misconceptions of mental illness. Hopefully, by educating disadvantaged populations, this could help reduce the stigma associated with mental health.

\section{Policy}

The mission of the social work profession is to serve the human needs of all people and empower the oppressed and vulnerable populations to thrive by providing culturally appropriate services to empower them to seek and access services in order to become productive citizens within the community. As social workers, it is within the profession to fight for social injustice and advocate for social change on behalf of clients. As a result, this dissertation brings forth a social problem within a vulnerable and oppressed population to enhance the well-being of the Mong/Hmong population in this country. Mong/Hmong children and youth are significantly underrepresented in the children's mental health system. With the increase of mental health illnesses and lack of mental health utilization within the Mong/Hmong community, it is crucial that local, state, and federal authorities make an initiative to address this growing issue. This research shows a need for policy makers to develop a culturally appropriate mental health committee to meet with Mong/Hmong Shaman/Shawoman, community leaders, and practitioners to exchange cultural knowledge and beliefs of physical, behavioral, and mental health illnesses; thus, developing strategies to implement culturally and appropriate education to deliver facts and misconception of illnesses within both cultures 
(i.e., Mong/Hmong and western). It is evident that community outreach and education are greatly needed to reduce the stigma associated with behavioral and mental health.

There are several key current political and conceptual challenges related to the social problem of Mong/Hmong adolescent suicide. First, this social problem has not been fully researched and there remains practically no available empirical data on the rate of Mong/Hmong adolescent or youth suicide attempts. Second, this social problem remains a minimal agenda for the local, state, and federal mental health systems as no policy or practice improvements have been implemented to better serve this ethnic group or the Southeast Asian population. Third, at the societal level, discrimination exists; therefore, the resistance of local, state, and federal authorities to change the delivery of mental health systems for ethnic minorities is problematic. It is evident that when compared to White European American youth, people of color have been overlooked throughout the history of the mental health system (Gibbs \& Huang, 1998). Often these minority children and youth lack the opportunity to obtain accessible mental health services, health care, community resources, and educational needs to maintain appropriate daily functioning. U.S. policies affecting youth in the mental health, education, child welfare, and juvenile justice systems have lacked the cultural sensitivity that minority groups need in order to prevail in this capitalist society (Gibbs \& Huang, 1998).

Another key point in improving policy and practice is that access to culturally competent mental health treatment has been an obstacle for many Mong/Hmong. For instance, Sue indicated (as cited in Gibbs \& Huang, 1998) that the mental health system 
lacks adequate culturally competent services, often resulting from limited research and researchers, practitioners, and policy makers often operating "without much knowledge of the issues" surrounding cultural groups' values, traditional beliefs, and histories (p. 1).Therefore, mental health policy makers must further encourage and support a multicultural mental health movement in delivering culturally competent services to ethnic minority clients. As can be seen in the mental health system history, government officials have made steady progress in incorporating cultural competency into policies, yet the extent of serving the needs of ethnic minorities remains limited. Lastly, cultural assessment should be inclusive of the Southeast Asian ethnicity, as most assessments have been predominately measured and assessed on minority groups such as African and Hispanic Americans (Gibbs \& Huang, 1998).

\section{Recommendations for Future Research}

This research study examined the psychological, social, and cultural factors that may affect suicide risk among Mong/Hmong youth between the ages of 18 and 25 . Results indicated a need to further explore the mental health of Mong/Hmong youth and explore available outpatient psychiatric clinical services to decrease depression and suicidal risk. The mental health research on Mong/Hmong youth remains limited as conducting rigorous empirical research on the prevalence of behavioral and mental health needs among Asian Americans has been a challenge for many researchers. For instance, Fugita observed (as stated in Serafica et al., 1990) that when researchers attempt to use standardized measurements on Asian Americans, they face the challenge of participants misinterpreting questions; that is, Asian Americans "respond differently to 
decontextualized instruments than western subjects" (p. 69). Asians have a greater difficulty responding to abstract questions and are likely to "focus more on the evaluative potential of their responses" (Serafica et al., 1990, p. 69).

Presently, there remains an insufficient number of research studies on the mental health needs of Southeast Asian youth, particularly the Mong/Hmong (DHHS, 1999). Of the current research studies on the Mong/Hmong population, more have focused on the mental health needs of older adults rather than children and young adults (Boehnlein \& Kinzie, 1996; R. C. Chung \& Lin, 1994; Mouanoutoua \& Brown, 1995; Nicholson, 1997; Ying \& Akursu, 1997). Furthermore, the availability and development of culturally sensitive and appropriate measurements for the Mong/Hmong population is scarce. According to Yee (n.d.), "Research to ensure that assessment and measurement tools are culturally competent for the Southeast Asian population is just beginning” (p. 10). Currently, there are only two well established and validated translated measurements for depression in the Mong/Hmong language (Mouanoutoua \& Brown, 1995; Mouanoutoua, Brown, Cappelletty, \& Levine, 1991).

It is evident there is a need to develop and conduct ongoing mental health research on this population, specifically with adolescents and young adults. Interestingly, this study indicated that depression and having a belief in Mong/Hmong traditional and spiritual healing practices are predictors of suicide risk among the sampled population; therefore, it is recommended that further mental health research on this population focus on developing robust culturally sensitive instruments to better measure how Mong/Hmong religious or cultural practices influence mental health. Additionally, it 
would be of interest for future researchers to consider conducting a qualitative study on suicide risk among those who have attempted suicide in order to explore and gain a better understanding of underlying factors and motivations to suicide. 


\section{References}

Agresti, A., \& Finlay, B. (1997). Statistical methods for the social sciences (3rd ed.). Upper Saddle River, NJ: Prentice-Hall.

Aldwin, C., \& Greenberger, E. (1987). Cultural differences in the predictors of depression. American Journal of Community Psychology, 15(6), 789-813.

American Psychiatric Association. (2000). Diagnostic and statistical manual of mental disorders (4th ed., text rev.). Washington, DC: Author.

Arnett, J. J. (2000). Emerging adulthood: A theory of development from the late teens through the twenties. American Psychologist, 55(5), 469-480.

Barrett, P. M., Sonderegger, R., \& Sonderegger, N. L. (2002). Assessment of child and adolescent migrants to Australia: A crosscultural comparison. Behaviour Change, 19, 220-235.

Beardslee, W. R., Gladstone, T. R., Wright, E. J., \& Cooper, A. B. (2003). A familybased approach to the prevention of depressive symptoms in children at risk: Evidence of parental and child change. Pediatrics, 112(2), 119-150.

Bearman, R. S. (1991). The social structure of suicide. Sociological Forum, 6(3), 501524.

Becker, A. E., Burwell, R. A., Navara, K., \& Gilman, S. E. (2003). Binge eating and binge eating disorder in a small-scale, indigenous society: The view from Fiji. International Journal of Eating Disorders, 34, 423-431. 
Berry, J. W. (1993). Ethnic identity in plural society. In M. E. Bernal, \& G. P. Knights (Eds.), Ethnic identity: Formation and transmission among Hispanics and other minorities (pp. 271-296). Albany, NY: SUNY Press.

Biggs, J. T., Wylie, L. T., \& Ziegler, V. E. (1978). Validity of the Zung Self-Rating Depression Scale. British Journal of Psychiatry, 132, 381-385.

Bliatout, B. T. (2003). Social and spiritual explanations of depression and nightmares. In K. A. Culhane-Pera, D. E. Vawter, P. Xiong, B. Babbit, \& M. M. Solberg (Eds.), Healing by heart: Clinical and ethical case stories of Hmong families and western providers (pp. 209-215). Nashville, TN: Vanderbilt University Press.

Bliatout, B. T., Downing, B. T., Lewis, J., \& Yang, D. (1988). Handbook for teaching Mong/Hmong-speaking students. Sacramento, CA: Spilman.

Boehnlein, J. L., \& Kinzie, J. D. (1996). Psychiatric treatment of Southeast Asian refugees. Retrieved from http://www.ncptsd.org/publications/cq /v6/n1/boehnlei.html

The California Endowment. (2006). Site visit and working meeting with the mental health services oversight and accountability commission at the Fresno Center for New Americans (CPA/FCNA Site Visit and Working Meeting Mental Health Services TCE 0301-2006). Retrieved from http://archive.acf.hhs.gov/programs/orr/policy/s102-38att.htm

Cao, L., \& Novas, H. (1996). Everything you need to know about Asian-American history. New York, NY: Penguin Books. 
Center for Substance Abuse Treatment. (2009). Addressing suicidal thoughts and behaviors in substance abuse treatment (Treatment Improvement Protocol [TIP] Series, No. 50). Rockville, MD: Substance Abuse and Mental Health Services Administration. Retrieved from http://www.ncbi.nlm.nih.gov/books/NBK64022/ Centers for Disease Control and Prevention. (2012). Suicide: Facts at a glance. Retrieved from http://www.cdc.gov/ViolencePrevention /pdf/Suicide_DataSheet-a.pdf

Cerhan, J. U. (1990). The Mong/Hmong in the United States: An overview for mental health professionals. Journal of Counseling and Development, 69(1), 88-92.

Chan, S. (1994). Mong/Hmong means free: Life in Laos and America. Philadelphia, PA: Temple University Press.

Chandy, J. M., Blum, R. W., \& Resnick, M. D. (1996). Female adolescents with a history of sexual abuse: Risk outcome and protective factors. Journal of Interpersonal Violence, 11(4), 503-518. doi: 10.1177/088626096011004004

Chen, H., Guarnaccia, P. J., \& Chung, H. (2003). Self-attention as a mediator of cultural influences on depression. International Journal of Social Psychiatry, 49, 192-203.

Cheng, J. K. Y., Fancher, T. L., Ratanasen, M., Conner, K. R., Duberstein, P. R., Sue, S., \& Takeuchi, D. (2010). Lifetime suicidal ideation and suicide attempts in Asian Americans. Asian American Journal of Psychology, 1(1), 18-30. doi:

$10.1037 / \mathrm{a} 0018799$ 
Chun, K. M., Morera, O. F., Andal, J. D., \& Shewes, M. C. (2007). Conducting research with diverse Asian American groups. In F. T. L. Leong, A. Ebreo, L. Kinoshita, A. G. Inman, L. H. Yang, \& M. Fu (Eds.), Handbook of Asian American psychology (2nd ed., pp. 47-65). Thousand Oaks, CA: Sage.

Chung, I. (2004). The prevalence of mental health problems among Asian American adolescents and children: Symptoms and treatment issues. Paper presented at the Eleventh Health Conference on Neurology and Psychiatry, New York, NY.

Chung, R. C., \& Lin, K. (1994). Help-seeking behavior among Southeast Asian refugees. Journal of Community Psychology, 22(2), 109-120.

Chung, R. H., Kim, B. S., \& Abreu, J. M. (2004). Asian American Multidimensional Acculturation Scale: Development, factor analysis, reliability, and validity. Cultural Diversity and Ethnic Minority Psychology, 10(1), 66-80.

Committee on Cultural Psychiatry, Group for the Advancement of Psychiatry. (1989). Suicide and ethnicity in the United States. New York, NY: Brunner/Mazel.

The Cultural Orientation Project. (2004). The Hmong: An introduction to their history and culture. Retrieved from http://www.cal.org/co /Mong/Hmong/hlaos.html

De Leon, B., \& Mendez, S. (1996). Factorial structure of a measure of acculturation in a Puerto-Rican population. Educational and Psychological Measurement, 56, 155165. 
Dhossche, D. M. (2003). Does Durkheim's social theory of suicide apply more to assisted suicide than suicide? Retrieved from http://www.priory.com /psych/durkheim.htm

Durkheim, E. (1987). Suicide: A study in sociology (J. A. Spaulding \& G. Simpson, Trans.). London, England: Routledge \& Kegan Paul. (Original work published 1951)

Eisenberg, D., Gollust, S. E., Golberstein, E., \& Hefner, J. L. (2007). Prevalence and correlates of depression, anxiety and suicidality among university students. American Journal of Orthopsychiatry, 77(4), 534-542.

Elder, J. P., Broyles, S. L., Brennan, J. J., Zuniga de Nuncio, M. L., \& Nader, P. R. (2005). Acculturation, parent-child acculturation differential, chronic disease risk factors in a Mexican-American population. Journal of Immigrant Health, 7, 1-9.

Eldering, L. (1995). Child-rearing in bi-cultural settings: A culture-ecological approach. Psychology Development Societies, 7(2), 132-153.

Ellis, A. D. (2002, August 11). Special report: Lost in America. Fresno Bee, pp. 12.

Erikson, E. H. (1950). Childhood and society (2nd ed.). New York, NY: W. W. Norton.

Erikson, E. H. (1968). Identity: Youth and crisis. New York, NY: W. W. Norton.

Fadiman, A. (1997). The spirit catches you and you fall down: A Hmong child, her American doctors, and the collision of two cultures. New York, NY: Farrar, Straus and Giroux.

Farver, J. M., Xu, Y., Bhadha, B. R., Narang, S., \& Lieber, E. (2007). Ethnic identity, acculturation, parenting beliefs, and adolescent adjustment: A comparison of 
Asian Indian and European American families. Merrill-Palmer Quarterly, $53(2), 184-215$.

Foss, G. F. (2001). Maternal sensitivity, posttraumatic stress, and acculturation in Vietnamese and Hmong mothers. The American Journal of Maternal/Child Nursing, 26, 257-263.

Fu, M. (2002). Acculturation, ethnic identity and family conflict among first and second generation Chinese Americans (Unpublished doctoral dissertation). Alliant International University, Alhambra, CA.

Garroutte, E. M., Goldberg, J., Beals, J., Herrell, R., Manson, S. M., \& Team, A. S. (2003). Spirituality and attempted suicide among American Indians. Social Science \& Medicine, 56(7), 1571-1579.

Gencoz, T., \& Or, P. (2006). Associated factors of suicide among university students: Importance of family environment. Contemporary Family Therapy, 28, 261-268.

Gibbs, J. T., \& Huang, L. N. (1998). Children of color: Psychological interventions with culturally diverse youth. San Francisco, CA: Jossey-Bass.

Gong-Guy, E. (1987). Report on the California Southeast Asian Mental Health Needs Assessment. Sacramento, CA: Department of Mental Health.

Gonzalez, G. M., \& Shriver, C. (2004). A bilingual computerized voice-interactive system for screening depression symptoms. Journal of Technology in Human Services, 22, 1-20. 
Greenberger, E., \& Chen, C. (1996). Perceived family relationships and depressed mood in early and late adolescence: A comparison of European and Asian Americans. Development Psychology, 32, 707-716.

Gutierrez, P. M., Osman, A., Kopper, B. A., Barrios, F. X., \& Bagge, C. L. (2000). Suicide risk assessment in a college student population. Journal of Counseling Psychology, 47, 403-413.

Hall, J. A. (1987). Parent-adolescent conflict: An empirical review. Adolescence, 12(88), 767-789.

Harachi, T. W., Catalano, R. F., Kim, S., \& Choi, Y. (2001). Etiology and prevention of substance use among Asian American youth. Prevention Science, 2(1), 57-65.

Hassan, R. (1998). One hundred years of Emile Durkheim's suicide: A study in sociology. Adelaide, Australia: Flinders University of South Australia.

Helzer, J. J. (1993). Maintenance of ethnic identity: Mong/Hmong and Mien settlement and survival in Northern California. Unpublished master's thesis, California State University, Chico, CA.

Huang, L. (2006). Sixteenth annual research conference highlights: Plenary sessions. Retrieved from http://rtckids.fmhi.usf.edu/rtcconference /16thconference /highlights.cfm

Huang, L. N., Lee, Y., \& Arganza, G. (2004). Promising approaches in youth development and risk prevention for Asian American/Pacific Islander youth: Voices from the field. Washington, DC: Georgetown University Center for Child and Human Development. 
Hyman, I., Vu, N., \& Beiser, M. (2000). Post-migration stresses among Southeast Asian refugee youth in Canada: A research note*. Journal of Comparative Family Studies, 31(2), 281-293.

Jesilow, P., \& Xiong, M. (2007). Constructing a social problem: Suicide, acculturation, and the Mong/Hmong. Mong/Hmong Studies Journal, 8, 1-43.

Juang, L. P., Nguyen, H. H., \& Lin, Y. (2006). The ethnic identity, other-group attitudes, and psychosocial functioning of Asian American emerging adults from two contexts. Journal of Adolescent Research, 21(5), 542-568.

Kang, H., Okazaki, S., Abelmann, N., Kim-Prieto, C., \& Lan, S. (2010). Redeeming immigrant parents: How Korean American emerging adults reinterpret their childhood. Journal of Adolescent Research, 25(3), 441-464.

Kenward, M. G., \& Carpenter, J. (2007). Multiple imputation: Current perspectives. Statistical Methods in Medical Research, 16, 199-218.

Kessler, R. C., Borges, C., \& Walters, E. E. (1999). Prevalence of and risk factors for lifetime suicide attempts in the National Comorbidity Survey. Archives of General Psychiatry, 56, 617-626.

Kim, B. S. K., \& Abreu, J. M. (2001). Acculturation measurement: Theory, current instruments, and future directions. In J. G. Ponterotto, J. M. Casas, L. A. Suzuki, \& C. M. Alexander (Eds.), Handbook of multicultural counseling (2nd ed., pp. 394-424). Thousand Oaks, CA: Sage.

Kisch, J., Leino, E. V., \& Silvermann, M. M. (2005). Aspects of suicidal behavior, depression, and treatment in college students: Results from the spring 2000 
National College Health Assessment Survey. Suicide and Life-Threatening Behavior, 35, 3-13.

Knight, R. G., Waal-Manning, J., \& Spears, G. F. (1983). Some norms and reliability data from the State-Trait Anxiety Inventory and the Zung Self-Rating Depression Scale. British Journal of Psychology, 22, 245-249.

Kvernmo, S., \& Heyerdahl, S. (2003). Acculturation strategies and ethnic identity as predictors of behavior problems in Arctic minority adolescents. Journal of the American Academy of Child \& Adolescent Psychiatry, 42(1), 54-65.

Lau, A. S., Jernewall, N. M., Zane, N., \& Meyers, H. F. (2002). Correlates of suicidal behaviors among Asian American outpatient youths. Cultural Diversity \& Ethnic Minority Psychology, 8, 199-213.

Lee, R. M., Choe, J., Kim, G., \& Ngo, V. (2000). Construction of the Asian American Family Conflicts Scale. Journal of Counseling Psychology, 42(2), 211-222.

Lee, R. M., Su, J., \& Yoshida, E. (2005). Coping with intergenerational family conflict among Asian American college students. Journal of Counseling Psychology, 52, 389-399.

Leenaars, A. A. (2008). Suicide: A cross-cultural theory. In M. M. Leach, \& F. T. L. Leong (Eds.), Suicide among racial and ethnic minority groups: Theory, research, and practice (pp. 13-37). New York, NY: Routledge/Taylor \& Francis.

Leong, C. W. (2005). Asian American parent-teen conflicts (Unpublished doctoral dissertation). St. John's University, New York. 
Lester, D. (2008). Theories of suicide. In M. M. Leach, \& F. T. L. Leong (Eds.), Suicide among racial and ethnic minority groups: Theory, research, and practice (pp. 3953). New York, NY: Routledge/Taylor \& Francis.

Little, R. J. A., \& Rubin, D. B. (2002). Statistical analysis with missing data (2nd ed.). New York, NY: John Wiley \& Sons.

Lo, B. (2009). Between two worlds: Mong/Hmong youth, culture, and socio-structural barriers to integration. U.C. Berkeley: Institute for the Study of Social Change. Retrieved from https://escholarship.org/uc/item/0518p675

Lorenzo, M. K., Pakiz, B., Reinherz, H. Z., \& Frost, A. (1995). Emotional and behavioral problems of Asian American adolescents: A comparative study. Child and Adolescent Social Work Journal, 12(3), 197-212.

MacDonald, J. L. (1997). Transnational aspects of Iu-Mien refugee identity. New York, NY: Routledge.

Marcell, A. V. (1994). Understanding ethnicity, identity formation, and risk behavior among adolescents of Mexican descent. Journal of School Health, 64(8), 323328.

Marcia, J. E. (1966). Development and validation of ego-identity status. Journal of Personality and Social Psychology, 3, 551-558.

Marcia, J. E. (1980). Identity in adolescence. In J. Adelson (Ed.), Handbook of adolescent psychology (pp. 159-187). New York, NY: Wiley.

Marsella, A. J., Kinzie, D., \& Gordon, P. (1973). Ethnic variations in the expression of depression. Journal of Cross-Cultural Psychology, 4, 435-458. 
McGinn, F. J. (1989). Hmong literacy among Hmong adolescents and the use of Hmong literacy during resettlement. Unpublished doctoral dissertation University of San Francisco, San Francisco, CA.

McInnis, K. (1991). Ethnic-sensitive work with Hmong refugee children. Child Welfare, $70,571-581$.

Messer, M. M., \& Rasmussen, N. H. (1986). Southeast Asian children in America: The impact of change. Pediatrics, 78(2), 323-329.

Miller, J. M., \& Lee, R. M. (2009). Factorial invariance of the Asian American Family Conflicts Scale across ethnicity, generational status, sex, and nationality. Measurement and Evaluation in Counseling and Development, 42, 179-196.

Mouanoutoua, V. L. (2003). Depression and posttraumatic stress disorder: Prevailing causes and therapeutic strategies with Hmong clients. In K. A. Culhane-Pera, D. E. Vawter, P. Xiong, B. Babbit, \& M. M. Solberg (Eds.), Healing by heart: Clinical and ethical case stories of Hmong families and western providers (pp. 216-221). Nashville, TN: Vanderbilt University Press.

Mouanoutoua, V. L., \& Brown, L. G. (1995). Hopkins Symptom Checklist-25, Hmong version: A screening instrument for psychological distress. Journal of Personality Assessment, 64(2), 376-383.

Mouanoutoua, V. L., Brown, L. G., Cappelletty, G. G., \& Levine, R. V. (1991). A Hmong adaptation of the Beck Depression Inventory. Journal of Personality Assessment, 57(2), 309-322. 
Muehlenkamp, J. J., Gutierrez, P. M., Osman, A., \& Barrios, F. X. (2005). Validation of the Positive and Negative Suicide Ideation (PANSI) Inventory in a diverse sample of young adults. Journal of Clinical Psychology, 61, 431-445.

National Alliance for the Mentally Ill. (2003). Asian Americans and Pacific Islander communities (AA/PIs) mental health facts. Retrieved from http://www.nami.org/Template.cfm?Section=Depression $\&$ Template=/ContentMa nagement/ContentDisplay.cfm\&ContentID=89034

National Asian American Pacific Islander Mental Health Association. (2007). Issues \& facts sheet. Retrieved from http://www.naapimha.org/issues /FactSheet.pdf

Nicholson, B. (1997). The influence of pre-emigration and post migration stressors on mental health: A study of Southeast Asian refugees. Social Work Research, 21(1), $13-33$.

Okazaki, S. (2002). Beyond questionnaires: Conceptual and methodological innovations for Asian American psychology. In N. Hall, \& S. Okazaki (Eds.), Asian American psychology: The science of lives in context (pp. 13-39). Washington, DC: American Psychological Association.

Oquendo, M. A., Dragatsi, D., Harkavy-Friedman, J., Dervic, K., Currier, D., Burke, A. K., . . Mann, J. J. (2005). Protective factors against suicidal behavior in Latinos. Journal of Nervous \& Mental Health Disease, 193(7), 438-443. 
Osman, A., Bagge, C. L., Guitierrez, P. M., Konick, L. C., Kooper, B. A., \& Barrios, F. X. (2001). The Suicidal Behaviors Questionnaire-Revised (SBQ-R): Validation with clinical and nonclinical sample. Assessment, 8(4), 443-454.

Pallent, J. (2005). SPSS survival manual (2nd ed.). New York, NY: Open University Press.

Patten, M. L. (2004). Understanding research methodology: An overview of the essentials (4th ed.). Glendale, CA: Pyrczak.

Pfaff, T. (1995). Mong/Hmong in America: Journey from a secret war. Eau Claire, WI: Chippewa Valley Museum Press.

Phinney, J. (1990). Ethnic identity in adolescents and adults: A review of research. Psychological Bulletin, 108, 499-514.

Phinney, J. (1992) The Multi-Group Ethnic Identity Measure: A new scale for use with adolescents and you adults from diverse groups. Journal of Adolescent Research, 7, 156-176.

Phinney, J. (2003). Ethnic identity and acculturation. In K. Chun, P. Ball, \& G. Marin (Eds.), Acculturation: Advances in theory, measurement, and applied research (pp. 63-81). Washington, DC: American Psychological Association.

Ponterotto, J. G., Baluch, S., \& Carielli, D. (1998). The Suinn-Lew Asian Self-Identity Acculturation Scale (SL-ASIA): Critique and research recommendations. Measurement and Evaluation in Counseling Development, 31, 109-124.

Quincy, K. (1988). Hmong: History of a people. Cheney, WA: Eastern Washington University Press. 
Quincy, K. (1995). Hmong: History of a people (2nd ed.). Cheney, WA: Eastern Washington University.

Radloff, L. S. (1977). The CES-D scale: A self-report depression scale for research in the general population. Applied Psychological Measurement, 1, 385-401.

Riley, A. W., \& Broitman, M. (2003). The effects of maternal depression on the school readiness of low-income children. Baltimore, MD: Report for the Annie E. Casey Foundation, Johns Hopkins Bloomberg School of Public Health.

Roberts, R. E., Phinney, J. S., Masse, L. C., Cheng, Y. R., Roberts, C. R., \& Romero, A. (1999). The structure of ethnic identity of young adolescents from diverse ethnocultural groups. Journal of Early Adolescence, 19, 301-322.

Rosenthal, D., Ranier, N., \& Klimidis, S. (1996). Vietnamese adolescents in Australia: Relationship between perceptions of self and parental values, intergenerational conflict, and gender dissatisfaction. Intergenerational Journal of Psychology, 31(2), 81-91.

Rubin, A., \& Babbie, E. (2001). Research methods for social work (4th ed.). Belmont, CA: Wadsworth/Thomson Learning.

Ryder, A. G., Alden, L. E., \& Paulhus, D. L. (2000). Is acculturation unidimensional or multidimensional? A head-to-head comparison in the prediction of personality, self-identity, and adjustment. Journal of Personality and Social Psychology, 79, 49-65. 
Saunders, J. A., Morrow-Howell, N., Spitznagel, E., Dore, P., Proctor, E. K., \& Pescarino, R. (2006). Imputing missing data: A comparison of methods for social work research. Social Work Research, 30, 19-30.

Serafica, F. C., Schwebel, A. I., Russell, R. K., Isaac, P. D., \& Myers, L. B. (1990). Mental health of ethnic minorities. New York, NY: Praeger.

Shrake, E. K., \& Rhee, S. (2004). Ethnic identity as a predictor of problem behaviors among Korean American adolescents. Adolescence, 39(155), 601-622.

Siegal, T., \& McSilver, J. (Producers). (2001). The split horn: Life of a Mong/Hmong shaman in America [Documentary]. United States: Alchemy Films.

Sluzki, C. (1979). Migration and family conflict. Family Process, 18, 379-390.

Snowden, L., \& Hines, A. M. (1999). A scale to assess African American acculturation. Journal of Black Psychology, 25, 36-47.

Snyder, H. N., \& Sickmund, M. (1999). Juvenile offenders and victims: 1999 national report (NCJ 178257). Washington, DC: U.S. Department of Justice, Office of Justice Programs, Office of Juvenile Justice and Delinquency Prevention.

Southeast Asia Resource Action Center. (2003, June). Southeast Asian Americans and higher education. Washington, DC: Author.

Stephenson, M. (2000). The development and validation of the Stephenson Multi-Group Acculturation Scale (SMAS). Psychological Assessment, 12, 77-88.

Su, J., Lee, R. M., \& Vang, S. (2005). Intergenerational family conflict and coping among Hmong American college students. Journal of Counseling Psychology, 52(4), 482-489. 
Sue, S. (2004). Overview: The assessment and treatment of Asian American families. In E. Lee (Ed.), Working with Asian Americans: A guide for clinicians (pp. 1-20). New York, NY: Guilford.

Suinn, R. M., Ahuna, C., \& Khoo, G. (1992). The Suinn-Lew Asian Self-Identity Acculturation Scale: Concurrent and factorial validation. Educational and Psychological Measurement, 52, 1041-1046.

Suinn, R. M., Ahuna, C., \& Khoo, G. (1995). The Suinn-Lew Asian Self-Identity Acculturation Scale: Cross-cultural information. Journal of Multicultural Counseling \& Development, 23(3), 139-150.

Tabachnick, B. G., \& Fidell, L. S. (2001). Using multivariate statistics (4th ed.). New York, NY: HarperCollins.

Tatman, A. W. (2004). Hmong history, culture, and acculturation: Implications for counseling the Hmong. Journal of Multicultural Counseling and Development, 24(3), 222-233.

Thao, C. (1999). The effect of Hmong students' perceptions, experience, and locus of control on college adjustment. Unpublished master's thesis, California State University, Chico, CA.

Thao, P., \& Yang, C. (2004). The Mong and the Mong/Hmong. Mong Journal, 1, 1-20.

Thao, Y. J. (2006). Culture and knowledge of the sacred instrument Qeej in the MongAmerican community. Asian Folklore Studies, 65, 249-267. 
Tsai, J., Ying, Y. W., \& Lee, P. A. (2001). Cultural predictors of self-esteem: A study of Chinese American female and male young adults. Cultural Diversity \& Ethnic Minority Psychology, 7, 284-297.

Tubergen, F., Grotenhuis, M., \& Ultee, W. (2005). Denomination, religious context, and suicide: Neo-Durkheimian multilevel explanations tested with individual and contextual data. American Journal of Sociology, 111(3), 797-823.

Uba, L. (1994). Asian Americans: Personality patterns, identity, and mental health. New York, NY: Guildford.

Um, K. (2003). A dream denied: Educational experiences of Southeast Asian American youth issues and recommendations. Washington, DC: Southeast Asia Resource Action Center.

U.S. Census Bureau. (2001). Census 2000. Retrieved from http://www.census.gov/population/www/cps/cpsdef.html

U.S. Department of Health and Human Services. (1999). Mental health: A report of the Surgeon General. Rockville, MD: Author.

U.S. Department of Health and Human Services. (2001). Mental health: Culture, race, and ethnicity - A supplement to mental health: A report of the Surgeon General. Rockville, MD: Author.

U.S. Department of Health and Human Services. (2002). Office of refugee resettlement: Executive summary. Retrieved from http://archive.acf.hhs.gov/programs/ orr/policy/s102-38att.htm 
U.S. Public Health Service. (1999). The Surgeon General's call to action to prevent suicide. Washington, DC: U.S. Department of Health and Human Services. Retrieved from http://www.surgeongeneral.gov /library/calltoaction/default.htm

Vangseng, V. (1995). Parent perceptions of education and participation in the schooling of Hmong children. Unpublished master's thesis, California State University, Chico, CA.

Walker-Moffat, W. (1995). The other side of the Asian American success story. San Francisco, CA: Jossey-Bass.

Westermeyer, J. (n.d.). PTSD among refugees. Retrieved from http://psydocfr.broca.inserm.fr/colloques/cr/apa96/apawestermeyer.pdf

Westermeyer, J., Tou, F. V., \& Neider, J. (1984). Symptom change over time among Hmong refugees: Psychiatric patients versus non-patients. Psychopathology, 17, 168-177.

World Health Organization. (2006). Effectiveness of brief intervention and contact for suicide attempters: A randomized controlled trial in five countries. Retrieved from http://www.who.int/bulletin/volumes/86/9/07-046995/en/

Worrell, F. C., Conyers, L. M., Mpofu, E., \& Vandiver, B. J. (2006). Multigroup Ethnic Identity Measure scores in a sample of adolescents from Zimbabwe. Journal of Theory and Research, 6(1), 35-59.

Yang, K. (2003). Mong/Hmong Americans: A review of felt needs, problems, and community development. Mong/Hmong Studies Journal, 4, 1-2. 
Yang, N. (1995). History and origin of the Hmong. Retrieved from http://www.Hmongcenter.org/briefhisofhm.html

Yee, B. W. K. (n.d.). Health and health care of Southeast Asian American elders: Vietnamese, Cambodians, Hmong and Laotian elders. Retrieved from http://www.stanford.edu/group/ethnoger/southeastasian.html

Ying, Y., \& Akursu, P. D. (1997). Psychological adjustment of Southeast Asian refugees: The contribution of sense of coherence. Journal of Community Psychology, 25(2), $125-139$.

Zane, N., \& Mak, W. (2003). Major approaches to the measurement of acculturation among ethnic minority populations: A content analysis and an alternative empirical strategy. In K. M. Chung, P. Balls Organista, \& G. Marin (Eds.), Acculturation: Advances in theory, measurement, and applied research (pp. 3690). Washington, DC: American Psychological Association.

Zung, W. W. (1965). A self-rating depression scale. Archives of General Psychiatry, 12, 63-70. 


\section{Appendix A: Informed Consent}

\section{The Role of Psycho-Sociocultural Factors in Suicide Risk Among Mong/Hmong Youth}

\section{CONSENT TO PARTICIPATE IN A RESEARCH STUDY}

You are invited to participate in a research study conducted by TangJudy Vang, A.S.W., Ph.D. Candidate from Portland State University (PSU), School of Social Work. The researcher hopes to learn about psycho-sociocultural factors that could influence depression and suicide risk among Mong/Hmong youth. This research study is being conducted in partial fulfillment of the requirements for a doctoral degree, under the supervision of Dr. Vikki Vandiver and Dr. Laurie Powers at PSU. The study is including 150 young adults, ages 18 to 25, who identify as being of Mong/Hmong ancestry and who live in California.

If you decide to participate, you will be asked to respond to a series of survey questionnaires. The survey will include questions about your age, gender and other areas such as your family, culture, ethnicity, and psychological well-being. The survey instruments take on average of 20 minutes to complete. While participating in this study, it is possible that you could feel some discomfort in answering some of the questions. Should the questions trigger some discomfort, please notify the researcher. You always have the options to skip the question(s) or withdraw from the study. Please be mindful that there is no penalty should you skip particular interview questions or withdraw from the study.

Your name or any other identifying information will not be written on your survey and your signed consent form will be stored in a locked file cabinet that is separated from your survey. Any information that you share with the researcher will be kept confidential with one exception. As a CA Associate Clinical Social Worker, this researcher is a mandated reporter; thus, should you disclose current thoughts of harming yourself or others, this researcher will have an ethical responsibility to contact the appropriate authorities in order to protect your health and the community. Everyone in the study will be given a list of community resources, and the researcher is available to help you connect to community resources, if you desire. You may not receive any direct benefit from taking part in this study, but the study may help to increase knowledge, which may help others in the future.

Your participation is voluntary. You do not have to take part in this study, and whether or not you take part in this study will not affect your course grades or other treatment by your academic institution. If you have questions about the study itself, please contact the researcher, TangJudy Vang at vangjudy@ hotmail.com or 916-320-6764, or her faculty adviser, Dr. Vikki Vandiver at 503-725-5007 or vandiverv@pdx.edu. If you have any concerns or problems about your participation in this study or your rights as a research 
subject, please contact the Human Subjects Research Review Committee, Office of Research and Sponsored Projects, 600 Unitus Bldg., Portland State University, (503) 725-4288 / 1-877-480-4400.

Your signature indicates that you have read and understand the above information and agree to take part in this study. Please understand that you may withdraw your consent at any time without penalty, and that by signing, you are not waiving any legal claims, rights or remedies. The researcher will provide you with a copy of this form for your own records.

Participant's Printed Name 


\section{Appendix B: Demographic Questionnaire DEMOGRAPHIC QUESTIONS ABOUT YOU}

Please tell me about you by placing a check $(\sqrt{ })$ next to the answer that best describe you:

1) I am:

— Male _ Female _ Transgender

1a) My sexual orientation is:

$\begin{array}{llll}- & \text { Heterosexual (e.g. straight) } & - & \begin{array}{c}\text { Homosexual (e.g. } \\ \text { gay, lesbian) }\end{array} \\ -\quad \text { Bisexual (e.g. both genders) } & - & \text { Not sure }\end{array}$

2) My age is:

3) I was born in (country):

- U.S.A. $\quad$ Thailand _ Other (please indicate):

4) My marital status is:

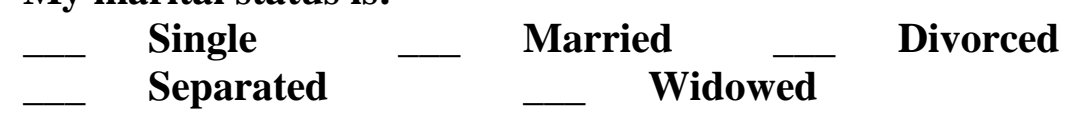

5) I live with:

No one (myself)

— Family member(s) (e.g. sister, brother, parents, husband, wife, extended family)

_ Friend(s)/Roommate(s)

— Significant partner (e.g. boyfriend, girlfriend)

6) My current college level is:

- Freshman

— Sophomore

- Junior

- Senior

— Graduate

7) I work this many hours a week:

$$
1 \text { to } 10
$$

10 to 20

20 to 30

30 to 40

- Unemployed 
8) My annual income is:

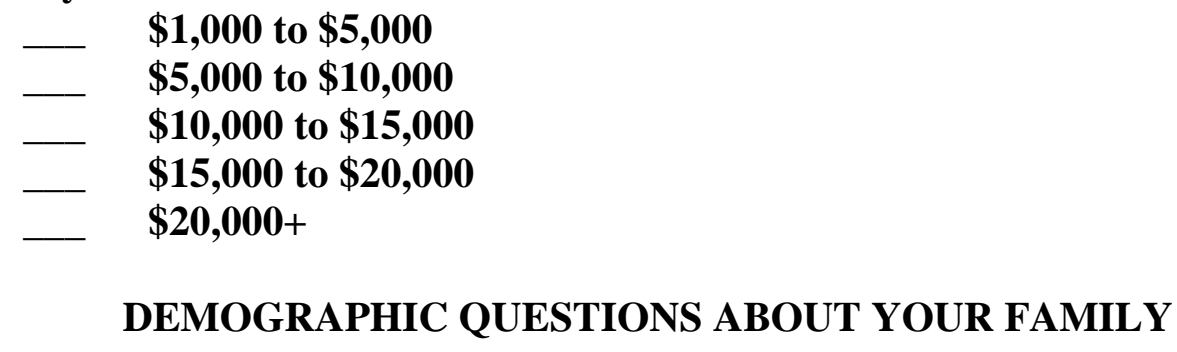

Please tell me about your family (the family that you were raised in, not your own) by placing a check $(\sqrt{ }$ ) next to the answer that best describe your family:

1) I was raised by:
Both biological parents
— One biological parent
_ Extended family members (e.g. aunt, uncle, cousins, etc.)
- Siblings

_ Other (please indicate):

2) My biological parents are:
- Married
- Divorced
— Separated
- Widowed
- Deceased
_ Other (please state):

3) My parents are:

_ Both U.S. citizens
— Only 1 is a U.S. citizen
- Not citizens

4) Including myself, my family household consists of:

$$
\begin{array}{ll}
-\quad 1 \text { to } 3 \\
-\quad 3 \text { to } 6 \\
-\quad 6 \text { to } 9 \\
-\quad 9 \text { to } 12 \\
-\quad 12+
\end{array}
$$


5) I was raised in a family in which:

Both parents legally worked

Only 1 parent legally worked

_ Both parents illegally worked ("under the table")

— Only 1 parent illegally worked ("under the table")

_ $\quad$ Relied on government assistance (e.g. welfare/TANF)

6) My family's estimated annual income is:

$-\$ 10,000$ to $\$ 20,000$

- $\$ 20,000$ to $\$ 30,000$

- $\$ 30,000$ to $\$ 40,000$

$-\$ 40,000+$

7) I live approximately minutes/hours from my family:

0 minute (I live with my family)

- 5 to 15 minutes

- 15 to 30 minutes

- 30 to 60 minutes

_ 1:00 to 2:00 hours

2:00 hours+ 


\section{Appendix C: Asian American Family Conflict Scale - Original}

\section{$\underline{\text { Asian American Family Conflict Scale - Original }}$}

The following statements are parent-child situations that may occur in your family. Consider how likely each situation occurs in your present relationship with your parents and how serious these conflicts are. Read each situation and answer the following questions using the following rating scales:

\begin{tabular}{|c|c|c|c|c|}
\hline 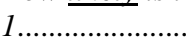 & 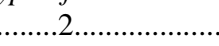 & 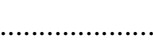 & ................. & .....5 \\
\hline Almost Never & Once in a While & Sometimes & Often or & $\begin{array}{l}\text { Almost Always } \\
\text { Frequently }\end{array}$ \\
\hline
\end{tabular}

How serious a problem is this situation in your relationship in your family?

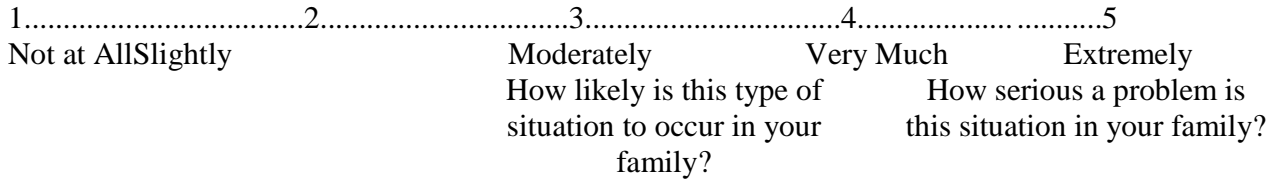

\section{Family Situations:}

1. Your parents tell you what to do with your life, but you want to make your own decisions.

2. Your parents tell you that a social lifeis not important at this age, but you think that it is.

3. You have done well in school, but yourparents academic expectations always exceed your performance.

4. Your parents want you to sacrifice personal interests for the sake of the family, but your feel this is unfair.

5. Your parents always compare you to others, but you want them to accept you for being yourself.

6. Your parents argue that they show you love by housing, feeding, and education you, but you wish they would show more physical and verbal signs of affection.

7. Your parents don't want you to bring shame upon the family, but you feel that your parents are too concerned with saving face.

8. Your parents expect you to behave like a proper Asian male or female, but you feel your parents are being too traditional.

9. You want to state your opinion, but your parents consider it to be disrespectful to talk back.

10. Your parents demand that you always show respect for elders, but you believe in showing respect only if they deserve it.

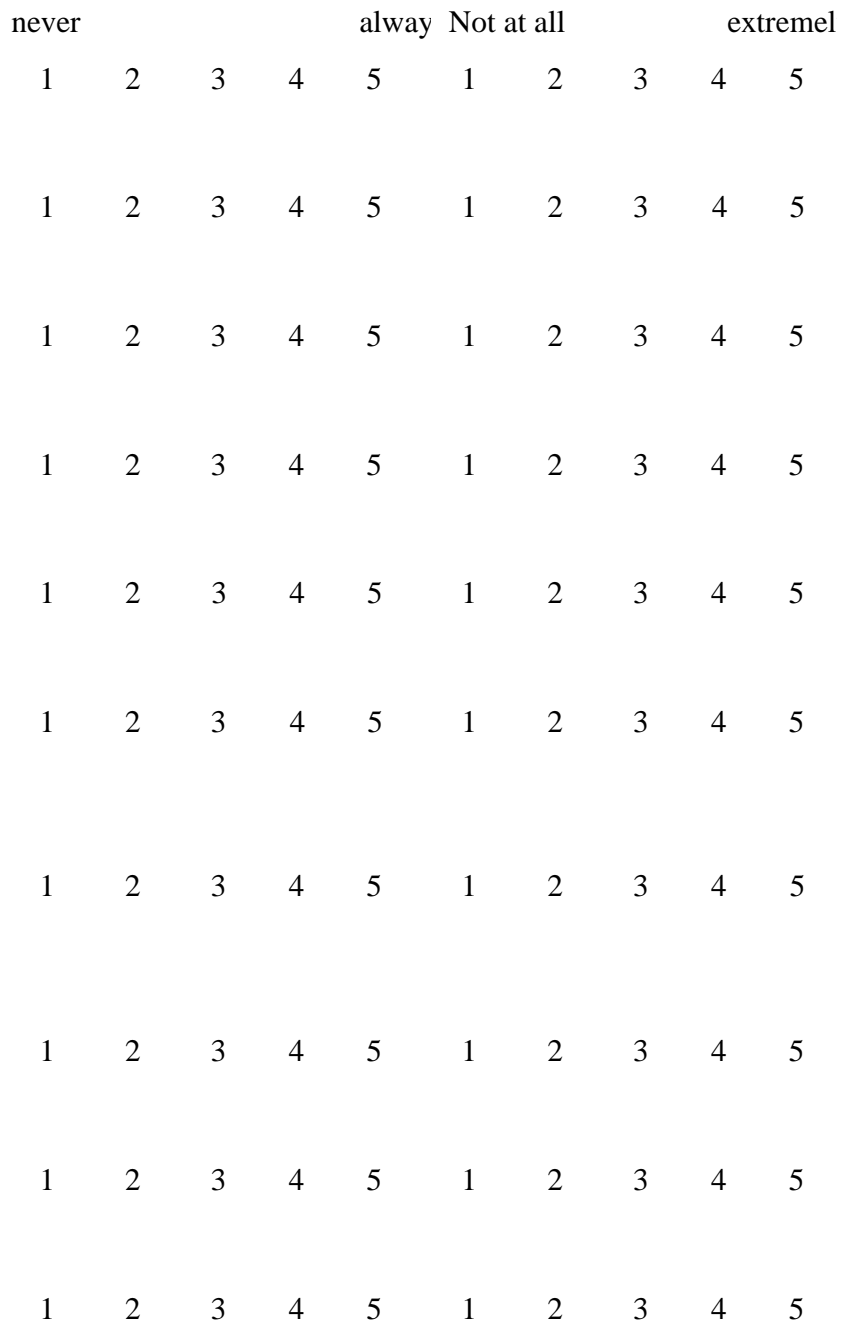

$\begin{array}{llllllllll}1 & 2 & 3 & 4 & 5 & 1 & 2 & 3 & 4 & 5\end{array}$ 


\section{Appendix D: Multigroup Ethnic Identity Measure \\ $\underline{\text { Multigroup Ethnic Identity Measure (MEIM) }}$}

In this country, people come from many different countries and cultures, and there are many different words to describe the different backgrounds or ethnic groups that people come from. Some examples of the names of ethnic groups are Hispanic or Latino, Black or African American, Asian American, Mong/Hmong, Chinese, Filipino, American Indian, Mexican American, Caucasian or White, Italian American, and many others. These questions are about your ethnicity or your ethnic group and how you feel about it or react to it.

\section{Please fill in: In terms of ethnic group, I consider myself to be}

Use the numbers below to indicate how much you agree or disagree with each statement.
(4) Strongly agree
(3) Agree
(2) Disagree
(1) Strongly disagree

1- __ I have spent time trying to find out more about my ethnic group, such as its history, traditions, and customs.

2- __ I am active in organizations or social groups that include mostly members of my own ethnic group.

3- __ I have a clear sense of my ethnic background and what it means for me.

4- __ I think a lot about how my life will be affected by my ethnic group membership.

5- __ I am happy that I am a member of the group I belong to.

6- __ I I have a strong sense of belonging to my own ethnic group.

7- __ I I understand pretty well what my ethnic group membership means to me.

8- __ In order to learn more about my ethnic background, I have often talked to other people about my ethnic group.

9- __ I have a lot of pride in my ethnic group.

10- __ I participate in cultural practices of my own group, such as special food, music, or customs.

11-___ I feel a strong attachment towards my own ethnic group. 
12- ___ I feel good about my cultural or ethnic background.

13- My ethnicity is (please circle one):

(1) Asian or Asian American, including Chinese, Japanese, Mong/Hmong, and others

(2) Black or African American

(3) Hispanic or Latino, including Mexican American, Central American, and others

(4) White, Caucasian, Anglo, European American; not Hispanic

(5) American Indian/Native American

(6) Mixed; Parents are from two different groups

(7) Other (write in):

14- __ My father's ethnicity is (use numbers above)

15-___ My mother's ethnicity is (use numbers above) 


\section{Appendix E: Suinn-Lew Asian Self-Identity Acculturation Scale SUINN-LEW ASIAN SELF-IDENTITY ACCULTURATION SCALE (SL-ASIA)}

INSTRUCTIONS: The questions which follow are for the purpose of collecting information about your historical background as well as more recent behaviors which may be related to your cultural identity. Choose the one answer which best describes you by circling the number next to the answer. For the purpose of this questionnaire, the term "Asian" is applied in a broad context which includes anyone who identifies their ethnic home country, ancestors, cultural background or characteristics from Asia (for example, Chinese, Mong/Hmong, Japanese, Korean, Iu-Mien, Vietnamese, etc.). The term "ethnic specific" is used to specifically identify one's ethic identity.

1. What language can you speak?

1. Asian only (for example, Chinese, Mong/Hmong, Japanese, Korean, Vietnamese, etc.)

2. Mostly Asian, some English

3. Asian and English about equally well (bilingual)

4. Mostly English, some Asian

5. Only English

2. What language do you prefer?

1. Asian only (for example, Chinese, Mong/Hmong, Japanese, Korean, Vietnamese, etc.)

2. Mostly Asian, some English

3. Asian and English about equally well (bilingual)

4. Mostly English, some Asian

5. Only English

3. How do you identify yourself?

1. Oriental

2. Asian

3. Asian-American

4. Ethnic specific (for example: Chinese-American, Hmong-American, Japanese American, Korean-American, etc.)

5. American

4. Which identification does (did) your mother use?

1. Oriental

2. Asian

3. Asian-American

4. Ethnic specific (for example: Chinese-American, Mong/Hmong, JapaneseAmerican, Korean-American, etc.)

5. American 
5. Which identification does (did) your father use?

1. Oriental

2. Asian

3. Asian-American

4. Ethnic specific (for example: Chinese-American, Mong/Hmong American, Japanese-American, Korean-American, etc.)

5. American

6. What was the ethnic origin of the friends and peers you had, as a child up to age 6 ?

1. Almost exclusively Asians, Asian-Americans

2. Mostly Asians, Asian-Americans

3. About equally Asian groups and Anglo groups

4. Mostly Anglos, Blacks, Hispanics, or other non-Asian ethnic groups

5. Almost exclusively Anglos, Blacks, Hispanics, or other non-Asian ethnic groups

7. What was the ethnic origin of the friends and peers you had, as a child from 6 to 18 ?

1. Almost exclusively Asians, Asian-Americans

2. Mostly Asians, Asian-Americans

3. About equally Asian groups and Anglo groups

4. Mostly Anglos, Blacks, Hispanics, or other non-Asian ethnic groups

5. Almost exclusively Anglos, Blacks, Hispanics, or other non-Asian ethnic groups

8. Whom do you now associate with in the community?

1. Almost exclusively Asians, Asian-Americans

2. Mostly Asians, Asian-Americans

3. About equally Asian groups and Anglo groups

4. Mostly Anglos, Blacks, Hispanics, or other non-Asian ethnic groups

5. Almost exclusively Anglos, Blacks, Hispanics, or other non-Asian ethnic groups

9. If you could pick, whom would you prefer to associate with in the community?

1. Almost exclusively Asians, Asian-Americans

2. Mostly Asians, Asian-Americans

3. About equally Asian groups and Anglo groups

4. Mostly Anglos, Blacks, Hispanics, or other non-Asian ethnic groups

5. Almost exclusively Anglos, Blacks, Hispanics, or other non-Asian ethnic groups

10. What is your music preference?

1. Only Asian music (for example, Chinese, Mong/Hmong, Japanese, Korean, Vietnamese, etc.)

2. Mostly Asian

3. Equally Asian and English

4. Mostly English

5. English only 
11. What is your movie preference?

1. Asian-language movies only (for example, Chinese, Mong/Hmong, Japanese, Korean, Vietnamese, etc.)

2. Asian-language movies mostly

3. Equally Asian/English English-language movies

4. Mostly English-language movies only

5. English-language movies only

12. What generation are you? (Circle the generation that best applies to you: )

1. 1 st Generation = I was born in Asia or country other than U.S.

2. 2nd Generation = I was born in U.S., either parent was born in Asia or country other than U.S.

3. 3rd Generation = I was born in U.S., both parents were born in U.S., and all grandparents born in Asia or country other than U.S.

4. 4th Generation = I was born in U.S., both parents were born in U.S., and at least one grandparent born in Asia or country other than U.S. and one grandparent born in U.S.

5. 5th Generation = I was born in U.S., both parents were born in U.S., and all grandparents also born in the U.S.

6. Don't know what generation best fits since I lack some information.

13. Where were you raised?

1. In Asia only

2. Mostly in Asia, some in U.S.

3. Equally in Asia and U.S.

4. Mostly in U.S., some in Asia

5. In U.S. only

14. What contact have you had with Asia (such as Thailand, Laos, Vietnam, China, etc.)?

1. Raised one year or more in Asia

2. Lived for less than one year in Asia

3. Occasional visits to Asia

4. Occasional communications (letters, phone calls, etc.) with people in Asia

5. No exposure or communications with people in Asia

15. What is your food preference at home?

1. Exclusively Asian food (for example, Chinese, Mong/Hmong, Japanese, Korean, Vietnamese, etc.)

2. Mostly Asian food, some American

3. About equally Asian and American

4. Mostly American food

5. Exclusively American food 
16. What is your food preference in restaurants?

1. Exclusively Asian food (for example, Chinese, Mong/Hmong, Japanese, Korean, Vietnamese, etc.)

2. Mostly Asian food, some American

3. About equally Asian and American

4. Mostly American food

5. Exclusively American food

17. Do you

1. Read only an Asian language? (for example, Chinese, Mong/Hmong, Japanese, Korean, Vietnamese, etc.)

2. Read an Asian language better than English?

3. Read both Asian and English equally well?

4. Read English better than an Asian language?

5. Read only English?

18. Do you

1. Write only an Asian language? (for example, Chinese, Mong/Hmong, Japanese, Korean, Vietnamese, etc.)

2. Write an Asian language better than English?

3. Write both Asian and English equally well?

4. Write English better than an Asian language?

5. Write only English?

19. If you consider yourself a member of the Asian group (Oriental, Asian, AsianAmerican, Chinese-American, Hmong-American, etc., whatever term you prefer), how much pride do you have in this group?

1. Extremely proud

2. Moderately proud

3. Little pride

4. No pride but do not feel negative toward group

5. No pride but do feel negative toward group

20. How would you rate yourself?

1. Very Asian (for example, Chinese, Mong/Hmong, Japanese, Korean, Vietnamese, etc.)

2. Mostly Asian

3. Bicultural

4. Mostly Westernized

5. Very Westernized 
21. Do you participate in Asian occasions, holidays, traditions, etc.? (for example, Hmong New Year celebrations, funeral rituals, healing ceremonies, name calling ceremonies, etc.)

1. Nearly all

2. Most of them

3. Some of them

4. A few of them

5. None at all

22. Rate yourself on how much you believe in Asian (for example, Mong/Hmong) values (e.g., about marriage, families, education, work):

$\begin{array}{llccc}1 & 2 & 3 & 4 & 5 \\ \text { (do not } & & & & \text { (strongly believe } \\ \text { believe) } & & & & \text { in Asian values) }\end{array}$

23. Rate yourself on how much you believe in American (Western) values:

$\begin{array}{llccc}1 & 2 & 3 & 4 & 5 \\ \text { (do not } & & & & \begin{array}{c}\text { (strongly believe } \\ \text { believe) }\end{array} \\ \text { in Asian values) }\end{array}$

24. Rate yourself on how well you fit when with other Asians of the same ethnicity:
1
2
3
(do not
4
5
(fit very well)

fit)

25. Rate yourself on how well you fit when with other Americans who are non-Asian (Westerners):
1
2
3
4
5
(do not
(fit very well)

fit)

26. There are many different ways in which people think of themselves. Which ONE of the following most closely describes how you view yourself? I consider myself basically:

1. An Asian person (e.g., Chinese, Mong/Hmong, Japanese, Korean, Vietnamese, etc.). Even though I live and work in America, I still view myself basically as an Asian person.

2. An American. Even though I have an Asian background and characteristics, I still view myself basically as an American.

3.An Asian-American, although deep down I always know I am an Asian.

4. An Asian-American, although deep down, I view myself as an American first.

5. An Asian-American. I have both Asian and American characteristics, and I view myself as a blend of both. 


\section{Appendix F: Zung Self-Rating Depression Scale}

Zung Self-Rating Depression Scale

Please read each statement and decide how much of the time the statement describes how you have been feeling during the past several days.

\begin{tabular}{|l|l|l|l|l|}
\hline \multicolumn{1}{|c|}{ Make check mark ( $\sqrt{\text { ) in appropriate }}$} & $\begin{array}{c}\text { A little of } \\
\text { the time }\end{array}$ & $\begin{array}{c}\text { Some of } \\
\text { the time }\end{array}$ & $\begin{array}{c}\text { Good part } \\
\text { of the time }\end{array}$ & $\begin{array}{c}\text { Most of } \\
\text { the time }\end{array}$ \\
\hline 1. I feel down-hearted and blue & & & & \\
\hline 2. Morning is when I feel the best & & & & \\
\hline 3. I have crying spells or feel like it & & & & \\
\hline 4. I have trouble sleeping at night & & & & \\
\hline 5. I eat as much as I used to & & & & \\
\hline 6. I still enjoy sex & & & & \\
\hline 7. I notice that I am losing weight & & & & \\
\hline 8. I have trouble with constipation & & & & \\
\hline 9. My heart beats faster than usual & & & & \\
\hline 10. I get tired for no reason & & & & \\
\hline 11. My mind is as clear as it used to be & & & & \\
\hline $\begin{array}{l}\text { 12. I find it easy to do the things I used } \\
\text { to }\end{array}$ & & & & \\
\hline 13. I am restless and can't keep still & & & & \\
\hline 14. I feel hopeful about the future & & & & \\
\hline 15. I am more irritable than usual & & & & \\
\hline 16. I find it easy to make decisions & & & & \\
\hline 17. I feel that I am useful and needed & & & & \\
\hline 18. My life is pretty full & & & & \\
\hline 19. I feel that others would be better off \\
if I were dead & & & & \\
\hline 20. I still enjoy the things I used to do & & & & \\
\hline
\end{tabular}




\section{Appendix G: SBQ-R Suicide Behavior Questionnaire Revised}

SBQ-R Suicide Behaviors Questionnaire-Revised

Instruction: Please check the number beside the statement or phrase that best applies to you.

\section{Have you ever thought about or attempted to kill yourself? (Check one only)}

1. $\square \quad$ Never

2. $\square \quad$ It was just a brief passing thought

3a. $\square \quad$ I have had a plan at least once to kill myself but did not try to do it

3b. $\square \quad$ I have had a plan at least once to kill myself and really wanted to die

4a. $\quad \square \quad$ I have attempted to kill myself, but did not want to die

4b. $\quad \square \quad$ I have attempted to kill myself, and really hoped to die

2. How often have you thought about killing yourself in the past year? (Check one only)
1. $\square \quad$ Never
2. $\square \quad$ Rarely (1 time)
3. $\square \quad$ Sometime (2 times)
4. $\square \quad$ Often (3-4 times)
5. $\quad \square \quad$ Very often (5 or more times)

3. Have you ever told someone that you were going to commit suicide, or that you might do it? (Check one only)

1. $\square \quad$ No

2a. $\square \quad$ Yes, at one time, but did not really want to die

2b. $\square \quad$ Yes, at one time, and really wanted to die

3a. $\square \quad$ Yes, more than once, but did not want to do it

3b. $\square \quad$ Yes, more than once, and really wanted to do it

4. How likely is it that you will attempt suicide someday? (Check one only)

$0 . \quad \square \quad$ Never

1. $\square \quad$ No chance at all

2. $\square \quad$ Rather unlikely

3. $\square \quad$ Unlikely

4. $\square \quad$ Likely

5. $\square \quad$ Rather likely

6. $\square \quad$ Very likely 


\section{Appendix H: Mong/Hmong Kev Ntseeg Instrument}

\section{Mong/Hmong Kev Ntseeg Instrument (MKNI)}

This instrument is designed to measure your spiritual orientation and your worldview of health.

1) My personal spiritual orientation is (check all that apply):

_ Animism/Shamanism (e.g. traditional Mong/Hmong ancestral worship)

- Christianity

_ Catholic

- Mormon

- Methodist

_ Other (please state):

2) My family's spiritual orientation is (check all that apply):

— Animism/Shan

Catholic

- Mormon

- Methodist

- Other (please state):

3) I have attended ceremonies with a Mong/Hmong shaman in the past year.

$$
0
$$

- $1-3$

- 4-6

- 7-9

_ More than 10 
Please read each statement and decide how much you agree with each statement.

Strongly

Disagree
4) I participate in traditional Mong/Hmong healing ceremonies (ua neeb, hu plig, ua nyuj dab, ua npua tai, pauj yeem, etc.) with either my family or friends.

5) I believe that all living beings, natural objects (e.g. rocks, water, air, etc.) and creatures are interconnected.

6) I believe that when a person dies, he/she is reborn into another human being, natural object (e.g. rocks, water, sand, etc.) or creature.

7) Physical illnesses are caused by spirits (e.g. ancestors or ntsui plig)

8) Thinking about negative things such as suicidality is caused by spirits (e.g. ancestors or ntsui plig)

9) If I have a physical illness, I would prefer to use a Mong/Hmong Shaman/Shawoman rather than seeing a western medical doctor

10) The best treatment to cure negative thinking such as suicidality is a Mong/Hmong Shaman/Shawoman

11) I believe that misfortunate is caused by spirits (e.g. ancestors or ntsui plig).

12) I believe that death may be caused by spirits (e.g. ancestors or ntsui plig).
Disagree

1

Neither Agree Agree Strongly

or Disagree

Agree

2

3

4

5

1

2

3

4

5

1

2

3

4

5

1

2

3

4

5

1

2

3

4

5

1

2

3

4

5

1

2

3

4

5

1

2

3

4

5

1

2 
Appendix I: Protective Factor Questions

The Role of Psycho-Sociocultural Factors in

Suicide Risk Among Mong/Hmong Youth

$\underline{\text { Protective Factors }}$

1) If you have had thoughts of ending your life, how did you respond to them (or deal with them)?

2) From your perspective, what would be most helpful to overcome having thoughts of ending one's life? 


\section{Appendix J: Research Questions \& Analyses}

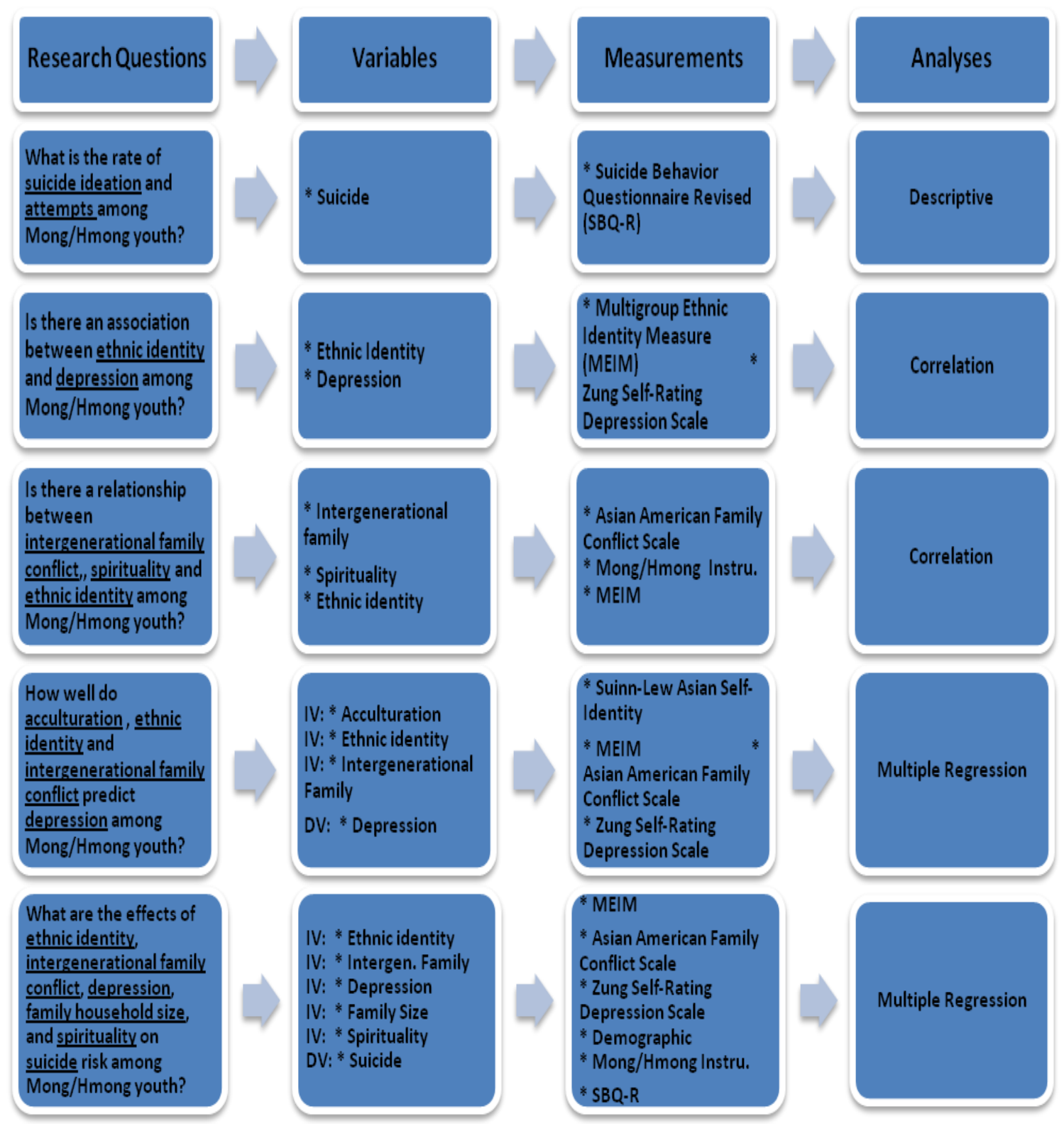




\section{Appendix K: Pilot Test}

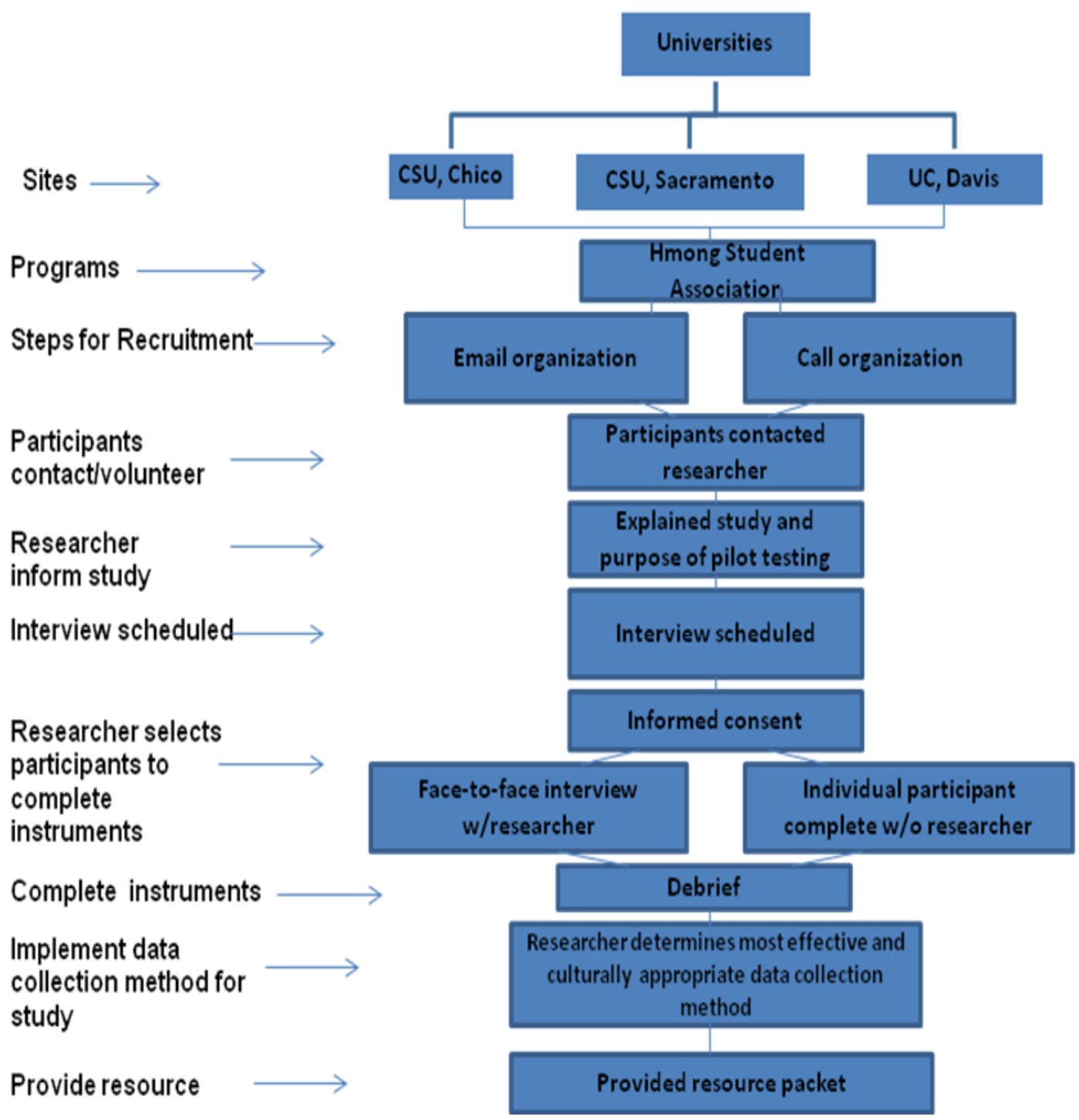




\section{Appendix L: Recruitment/Data Collection Process}

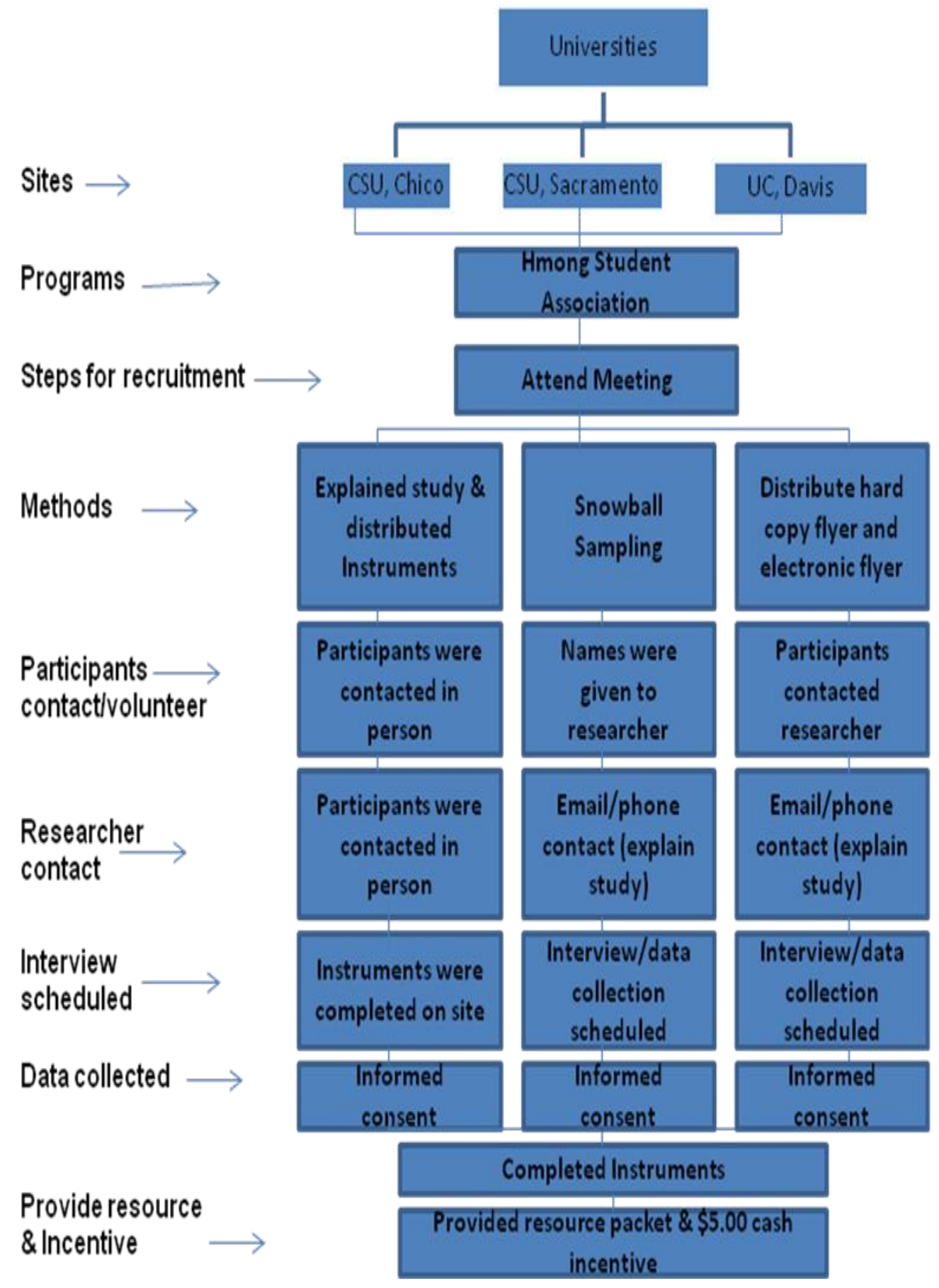

